\title{
New a priori and a posteriori probabilistic bounds for robust counterpart optimization: II. A priori bounds for known symmetric and asymmetric probability distributions
}

\author{
Yannis A. Guzman ${ }^{\mathrm{c}, \mathrm{a}, \mathrm{b}}$, Logan R. Matthews ${ }^{\mathrm{c}, \mathrm{a}, \mathrm{b}}$, Christodoulos A. Floudas ${ }^{\mathrm{a}, \mathrm{b}, *}$ \\ ${ }^{a}$ Artie McFerrin Department of Chemical Engineering, Texas A $\& M$ University, College \\ Station, TX, USA \\ ${ }^{b}$ Texas A $8 M$ Energy Institute, Texas A\&M University, College Station, TX, USA \\ ${ }^{c}$ Department of Chemical and Biological Engineering, Princeton University, Princeton, NJ, \\ $U S A$
}

\begin{abstract}
When optimization problems contain uncertain parameters, their nominal solutions may prove to be overly optimistic or even rendered infeasible given the actual parameter realizations. The application of probabilistic bounds in constructing the robust counterpart formulation of a model under uncertainty can greatly reduce the conservatism of traditional worst-case robust optimization. In Part I, we derived new a priori and a posteriori bounds on the probability of constraint violation for constraints with uncertain parameters whose distributions were unknown. Here, we first present new a priori bounds applicable to uncertain constraints with linearly participating uncertain parameters whose distributions are known or conservatively approximated. We then extend the robust counterpart optimization methodology by allowing attributed known distributions to be symmetric or asymmetric. The new methods greatly reduce the conservatism and significantly augment the performance and applicability of robust counterpart optimization. A mixed-integer linear optimization example and a multiperiod planning problem demonstrate the improvements of the new a priori bounds relative to existing bounds.
\end{abstract}

\section{Introduction}

In many applications, mathematical models contain parameters whose values are uncertain and cannot be known with absolute confidence. Whether these parameters represent selling prices, operation times, or supply and demand, their actual realizations can greatly alter the optimal solutions and objective function

\footnotetext{
*Corresponding author. Tel.: +1979 4580253.

Email address: floudas@tamu.edu (Christodoulos A. Floudas)
}

Preprint submitted to Elsevier

June 30, 2016

(C) 2016. This manuscript version is made available under the Elsevier user license http://www.elsevier.com/open-access/userlicense/1.0/ 
values of uncertain models, and can even render an optimal solution infeasible. Due to this, methods must be developed to ensure the feasibility of solutions for a variety of parameter values; the technique of robust counterpart optimization accomplishes this by guaranteeing the feasibility of all constraints over an uncertainty set which contains possible parameter realizations. The "worst-case" robust counterpart was developed by Soyster (1973), in which upper and lower bounds on each parameter are known and the uncertainty set for each constraint includes every possible parameter value. With bounded uncertainty, the probability that an optimal solution of the worst-case robust counterpart is rendered infeasible due to uncertain parameter realizations is zero.

The scope of robust optimization has greatly expanded over time. Uncertain least-squares optimization problems were addressed by El Ghaoui and Lebret (1997), and El Ghaoui et al. (1998) applied robust optimization to semidefinite optimization problems. As less conservative solutions are desired, uncertainty sets have been developed for models with bounded or unbounded uncertain parameters such that the probability of constraint violation is greater than zero. Ben-Tal and Nemirovski (2000) addressed bounded uncertainty in linear programs (LPs) using an interval + ellipsoidal uncertainty set, presenting an approach to provide an upper bound on the probability of constraint violation based on the size of the uncertainty set. Alternatively, an interval + polyhedral uncertainty set can be used that yields a linear robust counterpart, as developed by Bertsimas and Sim (2004). For uncertainty in mixed-integer linear programs (MILPs), Floudas and coworkers (Janak et al., 2007; Lin et al., 2004; Verderame and Floudas, 2009a,b) expanded the robust counterpart optimization framework. Other advances, highlighted elsewhere (Ben-Tal et al., 2009; Ben-Tal and Nemirovski, 2007; Bertsimas et al., 2011; Gabrel et al., 2014), have been achieved in adjustable robust optimization (Ben-Tal et al., 2003; Bertsimas and Goyal, 2012; Chen and Zhang, 2009) and theory regarding uncertainty sets and robust counterparts (Averbakh and Zhao, 2008; Ben-Tal et al., 2006; Bertsimas and Brown, 2009; Chen and Zhang, 2009).

The applications of robust optimization are broad in scope. They include areas such as design under uncertainty (Floudas et al., 2001), reactive scheduling (Janak et al., 2006), and scheduling of crude oil operations in a global optimization framework ( $\mathrm{Li}$ et al., 2012a); these applications include parameters subject to uncertainty with known or unknown probability distributions that could potentially be unbounded. Gounaris et al. (2013) proposed several robust optimization counterpart models for the capacitated vehicle routing problem under demand uncertainty. Lappas and Gounaris (2016) investigated process scheduling under uncertainty using a global event point-based MILP model, and proposed an approach based on adjustable multi-stage robust optimization that employs the heuristic of affine decision values. In a recent perspective article, Floudas et al. (2016) discussed the challenges and opportunities for addressing uncertainty via robust optimization methods in multi-scale systems engineering applications for energy and the environment.

It is important to note that the quality of robust solutions to uncertain LPs and MILPs relies heavily on the a priori or a posteriori probabilities of con- 
straint violation used to characterize them. In a priori methods, uncertainty sets are defined before a model is solved such that the uncertainty set satisfies an upper bound on the probability of constraint violation. For a given probability, a tighter a priori bound will yield a smaller uncertainty set, which includes a smaller range of uncertain parameter values to be considered. Thus, having tight a priori bounds will lead to less conservative and more appealing optimal solutions. A priori upper bounds on the probability of constraint violation based on the size of the uncertainty set have been developed by Ben-Tal and Nemirovski (2000) and Bertsimas and Sim (2004) for the interval + ellipsoidal and interval + polyhedral uncertainty sets, respectively. Kang et al. (2013) utilized the probability distribution of uncertain parameters to develop distributiondependent bounds for the interval + polyhedral set. Li et al. (2012b) extended these a priori bounds to other uncertainty sets, providing a variety of bounds applicable to the box, ellipsoidal, polyhedral, interval + ellipsoidal, and interval + polyhedral sets. Guzman et al. (2016a) proposed new a priori bounds for constraints with parameters subject to unknown probability distributions which are tighter than existing bounds (i.e., bounds proposed by Ben-Tal and Nemirovski, 2000; Bertsimas and Sim, 2004; Kang et al., 2013), and proposed the first bounds applicable to the case where distributions are unknown and their expected values are only known to exist within a range.

As a contrasting approach, a posteriori probabilistic guarantees were proposed that provide tighter bounds than their a priori counterparts but are calculated based on a particular solution (Guzman et al., 2016a; Kang et al., 2013; Li et al., 2012b; Li and Floudas, 2014; Paschalidis et al., 2008). These $a$ posteriori bounds can be incorporated directly into a robust counterpart; this only allows feasibility when the bound is met, but in general yields a nonconvex optimization problem ( $\mathrm{Li}$ and Floudas, 2014) which can be solved via stateof-the-art deterministic global optimization approaches such as GloMIQO or ANTIGONE (Gounaris et al., 2009; Misener and Floudas, 2010, 2012, 2013, 2014a,b; Misener et al., 2015, 2011). Alternatively, an iterative method was proposed to gain higher quality solutions by $\mathrm{Li}$ and Floudas (2014). Together, a priori and a posteriori bounds can drastically improve the solutions relative to the worst case or traditional one-pass approaches to robust optimization.

In Part I (Guzman et al., 2016a), we presented new a priori and a posteriori bounds on the probability of constraint violation for constraints with bounded, symmetric or asymmetric uncertain parameters with known or unknown expected values and unknown probability distributions. The work presented here builds upon the fundamental theoretical advancements of Part I and presents new a priori bounds for constraints with attributed probability distributions. Situations for which one or more of the new bounds are applicable include (i) bounded or unbounded, uncertain parameters with known symmetric probability distributions, and (ii) bounded or unbounded, uncertain parameters with known asymmetric probability distributions. Probability distributions can be rigorously modeled or conservatively attributed, and we discuss the significant improvements obtained when utilizing the uniform distribution to model unknown distributions. Computational examples demonstrate the reduced con- 
servatism of robust optimization when these new a priori bounds are utilized. The new a priori bounds address the prevailing conservatism issue in traditional one-pass robust optimization, leading to significantly better objective function values. The improvements become even more impactful when coupled with new a posteriori bounds to be presented in future work.

This article is structured as follows. Prerequisite theory on robust optimization is presented in Section 2. Section 3 contains derivations for the new $a$ priori bounds and provides illustrative examples on their usage. Implementation details for the new bounds are provided in Section 4. The new bounds are compared with existing methods over a number of computational case studies in Section 5. Concluding remarks are provided in Section 6, while additional reference material and auxiliary proofs are presented in the appendices.

\section{Background}

The contributions in this work are applicable to objective functions and constraints with uncertain parameters which participate linearly. Given continuous variables $x$ and integer variables $y$, consider inequality constraint $i$ which, possibly after reformulation, exhibits the form

$$
f_{i}(x, y)+\sum_{k} a_{i k} x_{k}+\sum_{\ell} b_{i \ell} y_{\ell}+\sum_{m} p_{i m} \leq 0
$$

where the exact value of some or all of the parameters $a_{i k}, b_{i \ell}$, and $p_{i m}$ are unknown, and $f_{i}(x, y)$ can be nonlinear. Without loss of generality, constraint (1) can be reformulated as:

$$
\begin{aligned}
f_{i}(x, y)+t_{i} & \leq 0 \\
-t_{i}+\sum_{k} a_{i k} x_{k}+\sum_{\ell} b_{i \ell} y_{\ell}+\sum_{m} p_{i m} & \leq 0 .
\end{aligned}
$$

Note that all uncertain parameters now participate in a linear inequality constraint. The same reformulation procedure can be applied to an objective function under uncertainty. Thus, we will assume that all constraints with uncertain parameters are linear with continuous or integer variables. The remaining material in this section is presented under the assumption that the entire model is an LP or MILP for simplicity.

\subsection{Modeling considerations}

The general form of an LP or MILP under uncertainty is as follows:

$$
\begin{array}{lll}
\max _{x, y} & \sum_{k} \tilde{c}_{k} x_{k}+\sum_{\ell} \tilde{d}_{\ell} y_{\ell} & \\
\text { s.t. } & \sum_{k} \tilde{a}_{i k} x_{k}+\sum_{\ell} \tilde{b}_{i \ell} y_{\ell} \leq \tilde{p}_{i} & \forall i \\
& y_{\ell} \in\{0,1\} & \forall \ell
\end{array}
$$


where any parameter denoted with a tilde, say $\tilde{p}$, denotes a parameter subject to uncertainty. Different realizations of the uncertain parameters can change the solution and objective function value of (3). Model (3) can be equivalently written (Li et al., 2012b) as:

$$
\begin{array}{lll}
\max _{x, y, z} & z & \\
\text { s.t. } & z-\sum_{k} \tilde{c}_{k} x_{k}-\sum_{\ell} \tilde{d}_{\ell} y_{\ell} \leq 0 & \\
& \tilde{p}_{i} x_{0}+\sum_{k} \tilde{a}_{i k} x_{k}+\sum_{\ell} \tilde{b}_{i \ell} y_{\ell} \leq 0 & \forall i \\
& x_{0}=-1 & \\
& y_{\ell} \in\{0,1\} & \forall \ell .
\end{array}
$$

An uncertain parameter $\tilde{a}$ can be represented by a constant nominal value $a$, which is generally set to $\mathbf{E}[\tilde{a}]$, and a random variable $\xi$ which subjects $a$ to perturbations weighted by a known positive constant $\hat{a}$ :

$$
\tilde{a}=a+\xi \hat{a} .
$$

Without loss of generality, models (3) and (4) can be represented as:

$$
\begin{array}{ll}
\max _{x} & \sum_{j} c_{j} x_{j} \\
\text { s.t. } & \sum_{j} a_{i j} x_{j}+\sum_{j \in J_{i}} \xi_{i j} \hat{a}_{i j} x_{j} \leq b_{i} \quad \forall i
\end{array}
$$

where $x_{j}$ is a continuous or integer variable, and $J_{i}:=\left\{j: \hat{a}_{i j}>0\right\}$ is the set of indices where the coefficients of $x$ in constraint $i$ are subject to uncertainty. The nominal value $a_{i j}$ of parameter $\tilde{a}_{i j}$ is typically chosen as $\mathbf{E}\left[\tilde{a}_{i j}\right]$. Given that $\tilde{a}_{i j}$ is subject to a bounded distribution, $\hat{a}_{i j}$ is typically chosen such that $\xi_{i j} \in[-1,1]$. If $\tilde{a}_{i j}$ is subject to an unbounded distribution, $\hat{a}_{i j}$ can be chosen to be the standard deviation of $\tilde{a}_{i j}$ so that $\xi_{i j}$ is standardized, that is, $\tilde{a}_{i j}=\mathbf{E}\left[\tilde{a}_{i j}\right]+\sigma_{i j} \xi_{i j}$, where $\sigma_{i j}$ is the standard deviation of $\tilde{a}_{i j}$, and the mean and variance of $\xi_{i j}$ is 0 and 1 , respectively.

\subsection{Uncertainty sets}

A nominal solution to (6) is one obtained when each uncertain parameter $\tilde{a}_{i j}$ is at its nominal value $a_{i j}$, that is, when $\xi_{i j}=0, \forall i, \forall j \in J_{i}$, while a robust solution will guarantee feasibility for more realizations of $\tilde{a}_{i j}$. The parameter space for which a robust solution is forced to guarantee feasibility is called an uncertainty set. We denote the uncertainty set of constraint $i$ as $U_{i}$, and define it in terms of random vector $\xi_{i}$ with elements $\xi_{i j}, j \in J_{i}$. For constraint $i, U_{i}$ is defined to contain some or all realizations of $\xi_{i}$, including the point $\xi_{i}=\mathbf{0}$ which corresponds to the nominal solution. $U_{i}$ is then imposed upon the feasible space 
of constraint $i$. The robust counterpart of model (6) can thus be written as

$$
\begin{array}{ll}
\max _{x} & \sum_{j} c_{j} x_{j} \\
\text { s.t. } & \sum_{j} a_{i j} x_{j}+\max _{\xi_{i} \in U_{i}}\left\{\sum_{j \in J_{i}} \xi_{i j} \hat{a}_{i j} x_{j}\right\} \leq b_{i} \quad \forall i .
\end{array}
$$

Generally, uncertainty sets are constructed geometrically by including parameter values within some norm distance of their nominal value. The simplest set, originally used by Soyster (1973) includes all possible values for bounded parameters in a box shape, forming the worst-case interval set. This can be generalized using the $\infty$-norm distance around the nominal value in order to change the size of the box, and is called the box uncertainty set (Li et al., 2011):

$$
U_{i}^{\infty}=\left\{\xi_{i}:\left\|\xi_{i}\right\|_{\infty}=\max _{j \in J_{i}}\left|\xi_{i j}\right| \leq \Psi_{i}\right\} .
$$

The worst-case set of Soyster (1973) corresponds to $\Psi_{i}=1$ and is known as the interval uncertainty set.

Similarly, the 1-norm or 2-norm can be used to define the uncertainty set, resulting in the polyhedral and ellipsoidal uncertainty sets, respectively (BenTal and Nemirovski, 1998, 1999, 2000; Bertsimas and Sim, 2004; El Ghaoui and Lebret, 1997; El Ghaoui et al., 1998; Li et al., 2011):

$$
\begin{aligned}
& U_{i}^{1}=\left\{\xi_{i}:\left\|\xi_{i}\right\|_{1}=\sum_{j \in J_{i}}\left|\xi_{i j}\right| \leq \Gamma_{i}\right\} \\
& U_{i}^{2}=\left\{\xi_{i}:\left\|\xi_{i}\right\|_{2}=\sqrt{\sum_{j \in J_{i}} \xi_{i j}^{2}} \leq \Omega_{i}\right\} .
\end{aligned}
$$

Note that when set parameters $\Psi_{i}, \Gamma_{i}$, and $\Omega_{i}$ equal zero, the corresponding uncertainty set reduces to the nominal parameter point; increasing the set parameters increases the size of $U_{i}$. Set parameters $\Psi_{i}, \Gamma_{i}$, and $\Omega_{i}$ are generically represented as $\Delta_{i}$ for an arbitrary uncertainty set $U_{i}$. If it is assumed that $\xi_{i j} \in[-1,1], \forall j \in J_{i}$, all possible parameter realizations are included in the box uncertainty set when $\Psi=1$ (i.e., the interval uncertainty set). Similarly, this occurs in polyhedral or ellipsoidal uncertainty sets when $\Gamma_{i}=\left|J_{i}\right|$ and $\Omega_{i}=\sqrt{\left|J_{i}\right|}$, respectively, where $\left|J_{i}\right|$ is the cardinality of set $J_{i}$ (i.e., $\left|J_{i}\right|$ is equal to the number of uncertain parameters in constraint $i$ ) (Li et al., 2011). However, due to the geometry of the uncertainty sets at these values of $\Gamma_{i}$ and $\Omega_{i}$, parameter realizations beyond those in the worst-case set are included. For bounded uncertainty, the interval + ellipsoidal (Ben-Tal and Nemirovski, 2000) and interval + polyhedral (Bertsimas and Sim, 2004) uncertainty sets address this through intersection of the interval and ellipsoidal sets and intersection of the interval and polyhedral sets, respectively:

$$
U_{i}^{2 \cap \infty}=\left\{\xi_{i}:\left\|\xi_{i}\right\|_{2} \leq \Omega_{i},\left\|\xi_{i}\right\|_{\infty} \leq 1\right\}
$$




$$
U_{i}^{1 \cap \infty}=\left\{\xi_{i}:\left\|\xi_{i}\right\|_{1} \leq \Gamma_{i},\left\|\xi_{i}\right\|_{\infty} \leq 1\right\} .
$$

Thus, the interval + ellipsoidal and interval + polyhedral sets are never larger than the worst-case set for bounded uncertainty. Table A.1 of Appendix A summarizes the five uncertainty sets considered in this work. The inclusion of uncertainty sets in robust counterpart formulations is summarized in Table A.2 of Appendix A and discussed in detail in (Li et al., 2011, 2012b; Li and Floudas, 2014).

\subsection{Probabilistic bounds}

If a model under uncertainty contains only bounded uncertain parameters, and if the model must remain feasible for any possible parameter realization, then the interval uncertainty set should be used for all uncertain constraints. However, it is often the case that a small, nonzero probability that constraint $i$ is infeasible can be permitted, or that some of the parameters are unbounded; the objective function value can then be significantly improved when compared to the worst-case solution. To do this, a probability of constraint violation, $\epsilon_{i}^{\text {prio }}$, is set before solving the model, representing an upper bound on the probability that constraint $i$ is violated in the optimal solution due to uncertain parameter realizations:

$$
\operatorname{Pr}\left\{\sum_{j} a_{i j} x_{j}+\sum_{j \in J_{i}} \xi_{i j} \hat{a}_{i j} x_{j}>b_{i}\right\} \leq \epsilon_{i}^{\text {prio }} .
$$

Knowing the desired $\epsilon_{i}^{\text {prio }}$, the size of set $U_{i}$ is chosen by selecting $\Delta_{i}$ values that are less than the worst-case value. Methods that relate $\epsilon_{i}^{\text {prio }}$ to $U_{i}$ are known as a priori probabilistic bounds. The value of $\Delta_{i}$ is chosen such that it is the smallest value, hence creating the smallest uncertainty set, that meets the desired bound on the probability of constraint violation. Formally, an optimal solution must be found to the following problem:

$$
\begin{array}{ll}
\min _{\Delta_{i}} & \Delta_{i} \\
\text { s.t. } & B\left(\Delta_{i}\right) \leq \epsilon_{i}^{\text {prio }} \\
& \Delta_{i} \geq 0
\end{array}
$$

where $B\left(\Delta_{i}\right)$ is an a priori bound expression. Solving (9) with a known, desired $\epsilon_{i}^{\text {prio }}$ will provide the necessary $\Delta_{i}$ for the robust counterpart formulation.

Once a robust counterpart is solved, the specific solution $x^{*}$ can be used to determine an upper bound $\epsilon_{i}^{\text {post }}$ on the probability that the solution will be infeasible in constraint $i$ with any parameter realization:

$$
\operatorname{Pr}\left\{\sum_{j} a_{i j} x_{j}^{*}+\sum_{j \in J_{i}} \xi_{i j} \hat{a}_{i j} x_{j}^{*}>b_{i}\right\} \leq \epsilon_{i}^{\text {post }} .
$$

Methods relating specific solutions of the robust counterpart to probabilities of constraint violation are known as a posteriori probabilistic bounds. 
The tightness of a priori or a posteriori bounds is of great relevance to optimal solutions; a tighter a priori bound for a specified $\epsilon_{i}^{\text {prio }}$ will provide a better objective function value, while a tighter a posteriori bound provides a lower value of $\epsilon_{i}^{\text {post }}$ for a known optimal solution than looser bounds. Generally, when more distributional information on the uncertain parameters is available, tighter bounds can be applied. The remainder of this section will discuss the recently proposed, best available bounds for bounded uncertain parameters whose distributions are completely unknown (see Guzman et al., 2016a) Uncertain parameters $\tilde{a}_{i j}$ are modeled as in (5) and with $\xi_{i j} \in[-1,1]$. The parameter $\delta_{i}^{(1)}$ depends on $U_{i}$ and is given in Table A.3 of Appendix A.

If there exist bounded uncertain parameters whose expected values are not known precisely, the only a priori bound available is (Guzman et al., 2016a):

$$
\begin{aligned}
& \operatorname{Pr}\left\{\sum_{j} a_{i j} x_{j}+\sum_{j \in J_{i}} \xi_{i j} \hat{a}_{i j} x_{j}>b_{i}\right\} \\
& \leq \exp \left(\min _{\theta>0}\left\{-\theta \Delta_{i}+\sum_{k \in K_{i}} \ln G_{i k}(\theta)\right\}\right)
\end{aligned}
$$

where $G_{i j}(\theta):=\mu_{i j} \sinh (\theta)+\cosh (\theta)$ and $K_{i}$ contains the indices of the $\left[\delta_{i}^{(1)}\right\rceil$ largest $\mu_{i j}$. Constants $\mu_{i j}$ and nominal values $a_{i j}$ are chosen such that $\left|\mathbf{E}\left[\xi_{i j}\right]\right| \leq$ $\mu_{i j}$. If the uncertainty set $U_{i}$ is an ellipsoidal or interval + ellipsoidal set, the following bound can improve the result of (GMF1) (Guzman et al., 2016a):

$$
\begin{aligned}
\operatorname{Pr}\left\{\sum_{j} a_{i j} x_{j}+\sum_{j \in J_{i}} \xi_{i j} \hat{a}_{i j} x_{j}>b_{i}\right\} \\
\leq \exp \left(\min _{\theta>0}\left\{-\theta \Delta_{i}+\left|J_{i}\right| \ln \bar{G}_{i}\left(\theta / \sqrt{\left|J_{i}\right|}\right)\right\}\right)
\end{aligned}
$$

where $\bar{\mu}_{i}=\max _{j \in J_{i}} \mu_{i j}$ and $\bar{G}_{i}(\theta):=\bar{\mu}_{i} \sinh (\theta)+\cosh (\theta)$.

When all the expected values of the uncertain parameters are known exactly, but the distributions remain completely unknown, the best available a priori bound for box, polyhedral, and interval + polyhedral sets is (Guzman et al., 2016a):

$$
\operatorname{Pr}\left\{\sum_{j} a_{i j} x_{j}+\sum_{j \in J_{i}} \xi_{i j} \hat{a}_{i j} x_{j}>b_{i}\right\} \leq\left(\alpha^{\alpha} \beta^{\beta}\right)^{-\delta_{i}^{(1)} / 2}
$$

where $\alpha:=1-\Delta_{i} / \delta_{i}^{(1)}$ and $\beta:=1+\Delta_{i} / \delta_{i}^{(1)}$. For ellipsoidal and interval + ellipsoidal sets, the best available bound is (Guzman et al., 2016a):

$$
\operatorname{Pr}\left\{\sum_{j} a_{i j} x_{j}+\sum_{j \in J_{i}} \xi_{i j} \hat{a}_{i j} x_{j}>b_{i}\right\} \leq\left(\alpha^{\alpha} \beta^{\beta}\right)^{-\left|J_{i}\right| / 2} .
$$


Finally, if the uncertain parameters belong to symmetric distributions, the best available bound for box, polyhedral, and interval + polyhedral sets is (Guzman et al., 2016a):

$$
\operatorname{Pr}\left\{\sum_{j} a_{i j} x_{j}+\sum_{j \in J_{i}} \xi_{i j} \hat{a}_{i j} x_{j}>b_{i}\right\} \leq \frac{1}{2^{\tilde{n}}} \sum_{k=\lceil\tilde{\nu}\rceil}^{\tilde{n}}\left(\begin{array}{l}
\tilde{n} \\
k
\end{array}\right)
$$

where $\tilde{\nu}:=\left(\Delta_{i}+\tilde{n}\right) / 2$ and

$$
\tilde{n}:= \begin{cases}\left|J_{i}\right|+1-\operatorname{sgn} \Delta_{i} & \text { if }\left\lceil\Delta_{i}\right\rceil+\left|J_{i}\right| \text { is even } \\ \left|J_{i}\right|-\operatorname{sgn} \Delta_{i} & \text { if }\left\lceil\Delta_{i}\right\rceil+\left|J_{i}\right| \text { is odd }\end{cases}
$$

where $\operatorname{sgn}(x)$ is the sign function which returns the sign of $x$. In certain situations, bound $\left(\mathrm{GMF}^{\prime}\right)$ might still outperform bound (GMF3) with ellipsoidal and interval + ellipsoidal sets.

In the determination of a priori probabilities of constraint violation, the following relations hold for the $i$ th constraint, any uncertainty set $U_{i}$, and any $\theta>0$, given that random variable $\xi_{i j}$ is independent for all $j \in J_{i}$ (Ben-Tal and Nemirovski, 2000; Bertsimas and Sim, 2004; Li et al., 2012b):

$$
\begin{aligned}
\operatorname{Pr}\left\{\sum_{j} a_{i j} x_{j}+\sum_{j \in J_{i}} \xi_{i j} \hat{a}_{i j} x_{j}>b_{i}\right\} & \leq \operatorname{Pr}\left\{\sum_{j \in J_{i}} \delta_{i j} \xi_{i j}>\Delta_{i}\right\} \\
& \leq e^{-\theta \Delta_{i}} \prod_{j \in J_{i}} \mathbf{E}\left[e^{\theta \delta_{i j} \xi_{i j}}\right] .
\end{aligned}
$$

The definitions of $\delta_{i j}$ are provided in Table A.3 of Appendix A and are dependent on the classification of uncertainty set $U_{i}$. As discussed in Li and Floudas (2014), each $\delta_{i j}$ is essentially $\hat{a}_{i j} x_{j}$ scaled by a factor dependent on $U_{i}$, and $\delta_{i j} \in[-1,1]$. All possible values of elements $\delta_{i j}, \forall j \in J_{i}$, of vector $\delta_{i}$ must be taken into account, and thus the value of $\delta_{i j}$ is not known a priori. The norm distances of $\delta_{i}$ are also constrained based on the type of uncertainty set $U_{i}$. All theorems in Section 3 use relations (11) and (12) as starting points. The theorems also make use of Lemmas 1 through 3, presented in Appendix B, which are mostly drawn from Part I (Guzman et al., 2016a).

\section{A Priori Bounds for Known Probability Distributions}

In contrast with Part I (Guzman et al., 2016a), in this paper it is assumed that the uncertain parameters are attributed to known probability distributions. This can be the result of rigorous modeling or the application of a distribution which, given an equivalent bounding method, would not yield better solutions than the true distribution. For example, a uniform probability distribution can often be attributed to bounded parameters with probability density con-

centrated about the mean and away from the tails; this is explored further in 
Section 5.1.1. Utilizing some typical distribution such as the uniform probability distribution still greatly decreases the conservatism when compared to utilizing the Rademacher distribution $\left(\operatorname{Pr}\left\{\xi^{(\mathrm{Ra})}=-1\right\}=0.5\right.$ and $\left.\operatorname{Pr}\left\{\xi^{(\mathrm{Ra})}=1\right\}=0.5\right)$ for a given random variable $\xi_{i j}$. Due to the results of Lemma 1, no conservatism is lost when attributing a Rademacher distribution to a (symmetric or asymmetric) bounded random variable $\xi_{i j}$, where $\xi_{i j} \in[-1,1]$ and $\mathbf{E}\left[\xi_{i j}\right]=0$, in the bounds that follow. Attributing Rademacher distributions to $\xi_{i j}, \forall j \in J_{i}$ will yield equivalent expressions to some of the bounds in Part I (Guzman et al., 2016a), where no distribution (nor symmetry) is assumed. If there is a case where the distributions of a subset of the uncertain parameters are bounded and have known means, but no other insights are possible, then attributing Rademacher distributions to those corresponding $\xi_{i j}$, attributing other distributions to the rest, and utilizing the bounds of this section will yield as good or better results than assuming all uncertain parameters are unknown as per Part I.

The bounds presented here require the existence of the moment generating function $M_{i j}(\theta)$ and the cumulant generating function $\Lambda_{i j}(\theta)$ of the attributed distribution of each random variable $\xi_{i j}$ :

$$
\begin{aligned}
M_{i j}(\theta) & :=\mathbf{E}\left[e^{\theta \xi_{i j}}\right] \\
\Lambda_{i j}(\theta) & :=\ln M_{i j}(\theta) .
\end{aligned}
$$

The moment generating function and derivatives of the cumulant generating function of common probability distributions are presented in Table A.4 of Appendix A. The bounds derived in this section are included in Tables 5 and 6; the first bounds assume symmetric probability distributions, and are followed by those which extend the methodology by accepting asymmetric probability distributions.

\subsection{Symmetric distributions}

Given cumulant generating functions $\Lambda_{i j}(\theta)$ for each symmetrically distributed random variable $\xi_{i j}$, Kang et al. (2013) derived the following bound for interval + polyhedral uncertainty sets:

$$
\begin{aligned}
\operatorname{Pr}\left\{\sum_{j} a_{i j} x_{j}+\sum_{j \in J_{i}} \xi_{i j} \hat{a}_{i j} x_{j}>b_{i}\right\} & \\
\leq & \exp \left(\min _{\theta>0}\left\{-\theta \Delta_{i}+\sum_{j \in J_{i}} \Lambda_{i j}(\theta)\right\} .\right.
\end{aligned}
$$

Bound (KBP1) is tight for polyhedral and interval + polyhedral uncertainty sets, but does not take into account $\delta_{i}^{(1)}$ or $\delta_{i}^{(2)}$, which can increase tightness for the other uncertainty sets. The new bounds presented in the sequel are similar in structure to (KBP1). For the purposes of the theorems in the sequel, the 
qualifying conditions of Lemmas 2 and 3 can be equivalently stated, respectively, in terms of $\Lambda_{i j}(\theta)$ as:

$$
\begin{array}{ll}
\frac{d \Lambda_{i j}(\theta)}{d \theta} \geq \frac{d \Lambda_{i k}(\theta)}{d \theta} & \forall j, k \in J_{i}, j<k, \forall \theta \geq 0 \\
\Lambda_{i j}(\sqrt{\theta}) \text { concave in } \theta & \forall \theta \geq 0 .
\end{array}
$$

Condition $\left(\mathrm{C}_{1}\right)$ enforces an ordering of the indices in $J_{i}$ where the relationships in $\left(\mathrm{C}_{1}\right)$ hold, while condition $\left(\mathrm{C}_{2}\right)$ qualifies the behavior of each cumulant generating function. Common distributions ordered by $\left(\mathrm{C}_{1}\right)$ and qualified by $\left(\mathrm{C}_{2}\right)$ are given in Table A.4 of Appendix A. Note that the cumulant generating function of a standard normal distribution meets condition $\left(\mathrm{C}_{2}\right)$ affinely, and that $\left(\mathrm{C}_{2}\right)$ is also met by the Rademacher distribution. As a consequence of Lemma 1, the moment generating function of the Rademacher distribution and its $n$th derivatives provide an upper bound on the moment generating functions and their $n$th derivatives of all symmetric distributions supported on $[-1,1]$ over $\theta \geq 0$. This fact coupled with our experience supports the conjecture that all bounded symmetric distributions supported on $[-1,1]$ meet condition $\left(\mathrm{C}_{2}\right)$; this is summarized below.

Conjecture 1. Given the random variable $\xi$ is subject to a symmetric probability distribution that is supported on the bounded interval $[-1,1]$, and given that its cumulant generating function $\Lambda_{\xi}(\theta)=\ln \mathbf{E}\left[e^{\theta \xi}\right]$ exists, then $\Lambda_{\xi}(\sqrt{\theta})$ is concave in $\theta$ over $\theta \in[0, \infty)$, that is, it meets condition $\left(\mathrm{C}_{2}\right)$.

Conjecture 1 would guarantee applicability of Corollaries 2.1 and 5.1, which are presented in the sequel and rely on condition $\left(\mathrm{C}_{2}\right)$, to all bounded and symmetric uncertain parameters. Note that all of the probability distributions in Table A.4, including those that are asymmetric, meet condition $\left(\mathrm{C}_{2}\right)$. These proofs are provided in Appendix C.

Theorem 1. Given that, for all $j \in J_{i}$, random variable $\xi_{i j}$ is independent and subject to a probability distribution that is symmetric about 0 , and given that it is possible to order the cumulant generating functions $\Lambda_{i j}(\theta)$ according to conditions $\left(\mathrm{C}_{1}\right)$, the following probability bound of constraint violation holds for the ith constraint and any $\theta>0$ :

$$
\begin{aligned}
\operatorname{Pr}\left\{\sum_{j} a_{i j} x_{j}+\sum_{j \in J_{i}} \xi_{i j} \hat{a}_{i j} x_{j}>b_{i}\right\} \\
\leq \exp \left(\min _{\theta>0}\left\{-\theta \Delta_{i}+\sum_{k \in K_{i}} \Lambda_{i k}(\theta)\right\}\right)
\end{aligned}
$$

where $K_{i}$ contains the first $\left\lceil\delta_{i}^{(1)}\right\rceil$ indices from the ordering in conditions $\left(\mathrm{C}_{1}\right)$. The parameter $\delta_{i}^{(1)}$ depends on $U_{i}$ and is given in Table A.3 of Appendix A. 
Proof.

$$
\begin{aligned}
\operatorname{Pr}\left\{\sum_{j} a_{i j} x_{j}+\sum_{j \in J_{i}} \xi_{i j} \hat{a}_{i j} x_{j}>b_{i}\right\} & \leq e^{-\theta \Delta_{i}} \prod_{j \in J_{i}} \mathbf{E}\left[e^{\theta \delta_{i j} \xi_{i j}}\right] \\
& \stackrel{1}{=} e^{-\theta \Delta_{i}} \prod_{j \in J_{i}} \mathbf{E}\left[e^{\theta\left|\delta_{i j}\right| \xi_{i j}}\right] \\
& =e^{-\theta \Delta_{i}} \prod_{j \in J_{i}} M_{i j}\left(\theta\left|\delta_{i j}\right|\right)
\end{aligned}
$$

where relation 1 is due to symmetry of the probability distribution of $\xi_{i j}$ about 0 . The optimization problem

$$
\max _{\left|\delta_{i}\right|}\left\{\prod_{j \in J_{i}} M_{i j}\left(\theta\left|\delta_{i j}\right|\right):\left\|\delta_{i}\right\|_{1} \leq \delta_{i}^{(1)} ;\left|\delta_{i j}\right| \in[0,1], \forall j \in J_{i}\right\}
$$

matches (P1) of Lemma 2; conditions (B.2) are satisfied due to given conditions $\left(\mathrm{C}_{1}\right)$. Then,

$$
\begin{aligned}
e^{-\theta \Delta_{i}} \prod_{j \in J_{i}} M_{i j}\left(\theta\left|\delta_{i j}\right|\right) & \stackrel{2}{\leq} e^{-\theta \Delta_{i}}\left(\prod_{k \in K_{i} \backslash\{\bar{j}\}} M_{i j}(\theta)\right) M_{i \bar{j}}\left(\theta\left[\delta_{i}^{(1)}-\left\lfloor\delta_{i}^{(1)}\right\rfloor\right]\right) \\
& \stackrel{3}{\leq} e^{-\theta \Delta_{i}}\left(\prod_{k \in K_{i} \backslash\{\bar{j}\}} M_{i j}(\theta)\right)\left(M_{i \bar{j}}(\theta)\right)^{\left(\delta_{i}^{(1)}-\left\lfloor\delta_{i}^{(1)}\right\rfloor\right)} \\
& =\exp \left(-\theta \Delta_{i}+\sum_{k \in K_{i} \backslash\{\bar{j}\}} \Lambda_{i j}(\theta)+\left(\delta_{i}^{(1)}-\left\lfloor\delta_{i}^{(1)}\right\rfloor\right) \ln \Lambda_{i \bar{j}}(\theta)\right) \\
& \stackrel{4}{\leq} \exp \left(-\theta \Delta_{i}+\sum_{k \in K_{i}} \Lambda_{i j}(\theta)\right)
\end{aligned}
$$

where relation 2 is due to Lemma 2, relation 3 is due to Jensen's inequality, and relation 4 is due to the inclusion of $\ln \Lambda_{i \bar{j}}(\theta)$ into the summation with a coefficient of 1 . The tightest bound is obtained by minimizing the expression over $\theta>0$.

Remark 1. Bound (GMF6) will always be as tight as or tighter than bound (KBP1) because it includes only a subset of the distributions in the calculation. For polyhedral and interval + polyhedral sets, the two bounds are equivalent.

Example 1. Consider the constraint

$$
\widetilde{1} x_{1}+\widetilde{1} x_{2} \leq \widetilde{20}
$$


where parameters $\widetilde{1}, \widetilde{1}$, and $\widetilde{20}$ are subject to bounded uncertainty. Each parameter can realize values within $10 \%$ of their expected values 1,1 , and 20 , respectively, and can be written as:

$$
\begin{aligned}
\widetilde{20} & =20+2 \xi_{0} \\
\widetilde{1} & =1+0.1 \xi_{1} \\
\widetilde{1} & =1+0.1 \xi_{2},
\end{aligned}
$$

where random variable $\xi_{j} \in[-1,1], j=0,1,2$. Constraint (13) can be reformulated as:

$$
\begin{aligned}
x_{1}+x_{2}+2 \xi_{0} x_{0}+0.1 \xi_{1} x_{1}+0.1 \xi_{2} x_{2} & \leq 20 \\
x_{0} & =-1 .
\end{aligned}
$$

The three uncertain parameters are modeled with uniform, triangular, and raised cosine distributions, respectively. Let us consider a probability of constraint violation of up to 0.1 using a box uncertainty set. The lowest value of $\Delta_{i}$ (that is, $\Psi$ ) will be found that guarantees this probability by utilizing bound (GMF6). Bound (GMF6) may exclude certain distributions after ordering them according to condition $\left(\mathrm{C}_{1}\right)$. The first index in the ordering will correspond to the distribution whose cumulant generating function's first derivatives are always greater than or equal to those of the rest over $\theta \geq 0$. The first derivatives of the cumulant generating function of the second index's distribution will be greater than or equal to those of subsequent indices over $\theta \geq 0$, and so on. In this case, all distributions are represented in Table A.4 of Appendix A, which provides the following ordering according to $\left(\mathrm{C}_{1}\right)$ : (1) uniform, (2) triangular, and (3) raised cosine. To use bound (GMF6) with a box uncertainty set, we set $\left|K_{i}\right|=\left\lceil\delta_{i}^{(1)}\right\rceil=1$, where $K_{i}$ only contains the index of the uniformly distributed random variable as per the ordering from condition $\left(\mathrm{C}_{1}\right)$. Based on (GMF6) and as per the form in (9), we must find an optimal solution to

$$
\begin{array}{ll}
\min _{\Psi} & \Psi \\
\text { s.t. } & \min _{\theta>0}\left\{-\theta \Psi+\ln \left(\theta^{-1} \sinh (\theta)\right)\right\} \leq \ln (0.1) \\
& \Psi \geq 0
\end{array}
$$

where $\ln \left(\theta^{-1} \sinh (\theta)\right)$ is the cumulant generating function of a uniform distribution supported on $[-1,1]$ (also provided in Table A.4 of Appendix A). We can efficiently determine the optimal value of $\Psi$ by using the bisection method (see Section 4 for details), which returns $\Psi=0.926$ and yields a probability of constraint violation of 0.1 :

$$
\exp \left(\min _{\theta>0}\left\{-0.926 \theta+\ln \left(\theta^{-1} \sinh (\theta)\right)\right\}\right)=\exp (-2.30)=0.10,
$$

where $\theta^{*}=13.514$. As per Table A.2 of Appendix A, the robust counterpart of the constraint with a box uncertainty set is:

$$
x_{1}+x_{2}+0.926\left(2+0.1\left|x_{1}\right|+0.1\left|x_{2}\right|\right) \leq 20 .
$$


Bound (GMF6) essentially eliminates the effect of certain distributions according to the ordering from condition $\left(\mathrm{C}_{1}\right)$. If condition $\left(\mathrm{C}_{1}\right)$ cannot be applied or its ordering is not easily determined, the maximization operator can be utilized as an alternative, as seen in Theorem 2 .

Theorem 2. Given that, for all $j \in J_{i}$, random variable $\xi_{i j}$ is independent and subject to a probability distribution that is symmetric about 0 , the following probability bound of constraint violation holds for the ith constraint and any $\theta>0$ :

$$
\begin{aligned}
& \operatorname{Pr}\left\{\sum_{j} a_{i j} x_{j}+\sum_{j \in J_{i}} \xi_{i j} \hat{a}_{i j} x_{j}>b_{i}\right\} \\
& \leq \exp \left(\min _{\theta>0}\left\{-\theta \Delta_{i}+\delta_{i}^{(1)} \max _{j \in J_{i}} \Lambda_{i j}(\theta)\right\}\right) .
\end{aligned}
$$

The parameter $\delta_{i}^{(1)}$ depends on $U_{i}$ and is given in Table A.3 of Appendix A.

Proof.

$$
\begin{aligned}
\operatorname{Pr}\left\{\sum_{j} a_{i j} x_{j}+\sum_{j \in J_{i}} \xi_{i j} \hat{a}_{i j} x_{j}>b_{i}\right\} & \leq e^{-\theta \Delta_{i}} \prod_{j \in J_{i}} \mathbf{E}\left[e^{\theta \delta_{i j} \xi_{i j}}\right] \\
& \stackrel{1}{=} e^{-\theta \Delta_{i}} \prod_{j \in J_{i}} \mathbf{E}\left[e^{\theta\left|\delta_{i j}\right| \xi_{i j}}\right] \\
& \leq e^{-\theta \Delta_{i}} \prod_{j^{\prime} \in J_{i}} \max _{j \in J_{i}} \mathbf{E}\left[e^{\theta\left|\delta_{i j^{\prime}}\right| \xi_{i j}}\right] \\
& =e^{-\theta \Delta_{i}} \prod_{j^{\prime} \in J_{i}} \max _{j \in J_{i}} M_{i j}\left(\theta\left|\delta_{i j^{\prime}}\right|\right)
\end{aligned}
$$

where relation 1 is due to symmetry of the probability distribution of $\xi_{i j}$ about 0 . The optimization problem

$$
\max _{\left|\delta_{i}\right|}\left\{\prod_{j^{\prime} \in J_{i}} \max _{j \in J_{i}} M_{i j}\left(\theta\left|\delta_{i j^{\prime}}\right|\right):\left\|\delta_{i}\right\|_{1} \leq \delta_{i}^{(1)} ;\left|\delta_{i j}\right| \in[0,1], \forall j \in J_{i}\right\}
$$

matches (P1) of Lemma 2; condition $\left(\mathrm{C}_{1}\right)$ and thus (B.2) are satisfied because the maximization operator yields equivalent functions within the product oper- 
ator. Then,

$$
\begin{aligned}
& e^{-\theta \Delta_{i}} \prod_{j^{\prime} \in J_{i}} \max _{j \in J_{i}} M_{i j}\left(\theta\left|\delta_{i j^{\prime}}\right|\right) \\
& \quad \stackrel{2}{\leq} e^{-\theta \Delta_{i}}\left(\max _{j \in J_{i}} M_{i j}(\theta)\right)^{\left\lfloor\delta_{i}^{(1)}\right\rfloor}\left(\max _{j \in J_{i}} M_{i j}\left(\theta\left[\delta_{i}^{(1)}-\left\lfloor\delta_{i}^{(1)}\right]\right]\right)\right) \\
& \stackrel{3}{\leq} e^{-\theta \Delta_{i}}\left(\max _{j \in J_{i}} M_{i j}(\theta)\right)^{\left\lfloor\delta_{i}^{(1)}\right\rfloor}\left(\max _{j \in J_{i}} M_{i j}(\theta)\right)^{\left(\delta_{i}^{(1)}-\left\lfloor\delta_{i}^{(1)}\right\rfloor\right)} \\
&=e^{-\theta \Delta_{i}}\left(\max _{j \in J_{i}} M_{i j}(\theta)\right)^{\delta_{i}^{(1)}} \\
&=\exp \left(-\theta \Delta_{i}+\delta_{i}^{(1)} \ln \max _{j \in J_{i}} M_{i j}(\theta)\right) \\
&=\exp \left(-\theta \Delta_{i}+\delta_{i}^{(1)} \max _{j \in J_{i}} \ln M_{i j}(\theta)\right)
\end{aligned}
$$

relation 2 is due to Lemma 2, and relation 3 is due to Jensen's inequality. The tightest bound is obtained by minimizing the expression over $\theta>0$.

Example 2. Consider the constraint

$$
\widetilde{1} x_{1}+\widetilde{2} x_{2} \leq \widetilde{12}
$$

where parameters $\widetilde{1}, \widetilde{2}$, and $\widetilde{12}$ are modeled by normal, Laplace, and normal distributions, respectively. Each parameter's nominal value represents its distribution's expected value, and the standard deviations of the distribution are $10 \%$ of their expected values. The parameters can be written as:

$$
\begin{aligned}
\widetilde{12} & =12+1.2 \xi_{0} \\
\widetilde{1} & =1+0.1 \xi_{1} \\
\widetilde{2} & =2+0.2 \xi_{2},
\end{aligned}
$$

where $\hat{a}_{j}$ for the unbounded parameters was chosen such that $\xi_{j}$ has unit variance. The set of indices $j$ associated with uncertain parameters in this constraint is $J=\{0,1,2\}$. Constraint (16) can be reformulated as:

$$
\begin{aligned}
x_{1}+2 x_{2}+1.2 \xi_{0} x_{0}+0.1 \xi_{1} x_{1}+0.2 \xi_{2} x_{2} & \leq 12 \\
x_{0} & =-1 .
\end{aligned}
$$

Let us consider a probability of constraint violation of up to 0.1 using a box uncertainty set. The lowest value of $\Delta_{i}$ (that is, $\Psi$ ) will be found that guarantees this probability by utilizing bound (GMF7). Only the largest cumulant generating function at a particular $\theta$ participates in bound (GMF7). Based on 
(GMF7) and as per the form in (9), we must find an optimal solution to

$$
\begin{array}{ll}
\min _{\Psi} & \Psi \\
\text { s.t. } & \min _{\theta>0}\left\{-\theta \Psi+\max \left\{\frac{\theta^{2}}{2},-\ln \left(1-\frac{\theta^{2}}{2}\right)\right\}\right\} \leq \ln (0.1) \\
& \Psi \geq 0
\end{array}
$$

where $\theta^{2} / 2$ is the cumulant generating function of a standard normal distribution, and $-\ln \left(1-\theta^{2} / 2\right)$ is the cumulant generating function of a standardized Laplace distribution (not to be confused with other variants, see (Kotz et al., 2012)). We can efficiently determine the optimal value of $\Psi$ by using the bisection method (see Section 4 for details), which returns $\Psi=2.937$ and yields a probability of constraint violation of 0.1 :

$$
\exp \left(\min _{\theta>0}\left\{-2.937 \theta+\max \left\{\frac{\theta^{2}}{2},-\ln \left(1-\frac{\theta^{2}}{2}\right)\right\}\right\}\right)=\exp (-2.30)=0.10
$$

where $\theta^{*}=1.114$. Note that it is acceptable for $\Psi$ to be greater than 1 when parameters are unbounded; when $\xi_{i j} \in[-1,1], \forall j \in J_{i}$, the interval (worstcase) uncertainty set uses $\Psi=1$. As per Table A.2 of Appendix A, the robust counterpart of the constraint with a box uncertainty set is:

$$
x_{1}+2 x_{2}+2.937\left(1.2+0.1\left|x_{1}\right|+0.2\left|x_{2}\right|\right) \leq 12 .
$$

Corollary 2.1. For ellipsoidal and interval + ellipsoidal uncertainty sets, given that $\Lambda_{i j}(\theta)$ satisfies condition $\left(\mathrm{C}_{2}\right)$ for all $\theta>0$, the following probability bound of constraint violation holds for the ith constraint and any $\theta>0$ :

$$
\begin{aligned}
\operatorname{Pr}\left\{\sum_{j} a_{i j} x_{j}\right. & \left.+\sum_{j \in J_{i}} \xi_{i j} \hat{a}_{i j} x_{j}>b_{i}\right\} \\
& \leq \exp \left(\min _{\theta>0}\left\{-\theta \Delta_{i}+\left|J_{i}\right| \max _{j \in J_{i}} \Lambda_{i j}\left(\theta / \sqrt{\left|J_{i}\right|}\right)\right\}\right) .
\end{aligned}
$$

Proof.

$$
\begin{aligned}
\operatorname{Pr}\left\{\sum_{j} a_{i j} x_{j}+\sum_{j \in J_{i}} \xi_{i j} \hat{a}_{i j} x_{j}>b_{i}\right\} & \leq e^{-\theta \Delta_{i}} \prod_{j \in J_{i}} \mathbf{E}\left[e^{\theta \delta_{i j} \xi_{i j}}\right] \\
& \underline{1} e^{-\theta \Delta_{i}} \prod_{j \in J_{i}} \mathbf{E}\left[e^{\theta\left|\delta_{i j}\right| \xi_{i j}}\right] \\
& \leq e^{-\theta \Delta_{i}} \prod_{j^{\prime} \in J_{i}} \operatorname{maxaJ}_{j \in J_{i}} \mathbf{E}\left[e^{\theta\left|\delta_{i j^{\prime}}\right| \xi_{i j}}\right] \\
& =\exp \left(-\theta \Delta_{i}+\sum_{j^{\prime} \in J_{i}} \max _{j \in J_{i}} \Lambda_{i j}\left(\theta\left|\delta_{i j^{\prime}}\right|\right)\right.
\end{aligned}
$$


where relation 1 is due to symmetry of the probability distribution of $\xi_{i j}$ about 0 . The optimization problem

$$
\max _{\left|\delta_{i}\right|}\left\{\sum_{j^{\prime} \in J_{i}} \max _{j \in J_{i}} \Lambda_{i j}\left(\theta\left|\delta_{i j^{\prime}}\right|\right):\left\|\delta_{i}\right\|_{2} \leq \delta_{i}^{(2)} ;\left|\delta_{i j}\right| \in[0,1], \forall j \in J_{i}\right\}
$$

matches (P2) of Lemma 3 due to the fact that $\delta_{i}^{(2)}=1$ for ellipsoidal and interval + ellipsoidal sets; the conditions of Lemma 3 are satisfied due to given conditions $\left(\mathrm{C}_{2}\right)$.

Then,

$$
\exp \left(-\theta \Delta_{i}+\sum_{j^{\prime} \in J_{i}} \max _{j \in J_{i}} \Lambda_{i j}\left(\theta\left|\delta_{i j^{\prime}}\right|\right)\right) \stackrel{2}{\leq} \exp \left(-\theta \Delta_{i}+\left|J_{i}\right| \max _{j \in J_{i}} \Lambda_{i j}\left(\theta / \sqrt{\left|J_{i}\right|}\right)\right)
$$

where relation 2 is due to Lemma 3 . The tightest bound is obtained by minimizing the expression over $\theta>0$.

Example 3. Consider the constraint

$$
\widetilde{1} x_{1}+\widetilde{2} x_{2} \leq \widetilde{12}
$$

where parameters $\widetilde{1}, \widetilde{2}$, and $\widetilde{12}$ are subject to bounded uncertainty. Each parameter can realize values within $10 \%$ of their expected values 1,2 , and 12 , respectively, and can be written as:

$$
\begin{aligned}
\widetilde{12} & =12+1.2 \xi_{0} \\
\widetilde{1} & =1+0.1 \xi_{1} \\
\widetilde{2} & =2+0.2 \xi_{2},
\end{aligned}
$$

where random variable $\xi_{j} \in[-1,1], j=0,1,2$. Constraint (19) can be reformulated as:

$$
\begin{aligned}
x_{1}+2 x_{2}+1.2 \xi_{0} x_{0}+0.1 \xi_{1} x_{1}+0.2 \xi_{2} x_{2} & \leq 12 \\
x_{0} & =-1 .
\end{aligned}
$$

The three uncertain parameters are modeled with uniform, triangular, and raised cosine distributions, respectively. Let us consider a probability of constraint violation of up to 0.1 using an interval + ellipsoidal uncertainty set. The lowest value of $\Delta_{i}$ (that is, $\Omega$ ) will be found that guarantees this probability by utilizing bound $\left(\mathrm{GMF}^{\prime}\right)$. Note that, as per Table A.4 of Appendix A, the first derivatives of the cumulant generating function of the uniform distribution are always greater than that of a symmetric triangular or raised cosine distribution. Thus, since all cumulant generating functions are 0 at $\theta=0$, the cumulant generating function of the uniform distribution is always greater than or equal 
to those of the other distributions, and thus replaces the $\max \{\}$ term. Based on $\left(\mathrm{GMF}^{\prime}\right)$ and as per the form in (9), we must find an optimal solution to

$$
\begin{array}{ll}
\min _{\Omega} & \Omega \\
\text { s.t. } & \min _{\theta>0}\left\{-\theta \Omega+3 \ln \left(\sqrt{3} \theta^{-1} \sinh (\theta / \sqrt{3})\right)\right\} \leq \ln (0.1) \\
& \Omega \geq 0
\end{array}
$$

where $\ln \left(\sqrt{3} \theta^{-1} \sinh (\theta / \sqrt{3})\right)$ is the cumulant generating function of a uniform distribution supported on $[-1,1]$ (also provided in Table A.4 of Appendix A) with the argument $\theta / \sqrt{3}$ as per $\left(\mathrm{GMF}^{\prime}\right)$. We can efficiently determine the optimal value of $\Omega$ by using the bisection method (see Section 4 for details), which returns $\Omega=1.139$ and yields a probability of constraint violation of 0.1 :

$$
\exp \left(\min _{\theta>0}\left\{-1.139 \theta+3 \ln \left(\sqrt{3} \theta^{-1} \sinh (\theta / \sqrt{3})\right)\right\}\right)=\exp (-2.30)=0.10
$$

where $\theta^{*}=4.964$. As per Table A.2 of Appendix A, the robust counterpart of the constraint with an interval + ellipsoidal set is:

$$
\begin{aligned}
x_{1}+2 x_{2}+1.2 \mid & -1-z_{0}|+0.1| x_{1}-z_{1} \mid \\
& +0.2\left|x_{2}-z_{2}\right|+1.139 \sqrt{1.44 z_{0}^{2}+0.01 z_{1}^{2}+0.04 z_{2}^{2}} \leq 12 .
\end{aligned}
$$

\subsection{Asymmetric distributions}

The bounds presented in Section 3.1 can be generalized for asymmetric distributions given the existence of the following parameter, $g_{i j}$, for each asymmetric probability distribution $\xi_{i j}$ :

$$
g_{i j}:=\left\{\begin{array}{lll}
+1 & \text { if } M_{i j}(\theta) \geq M_{i j}(-\theta) & \forall \theta \geq 0 \\
-1 & \text { if } M_{i j}(\theta) \leq M_{i j}(-\theta) & \forall \theta \geq 0
\end{array}\right.
$$

which permits the following inequality:

$$
\mathbf{E}\left[e^{\theta \delta_{i j} \xi_{i j}}\right] \leq \mathbf{E}\left[e^{g_{i j} \theta\left|\delta_{i j}\right| \xi_{i j}}\right] .
$$

The term $\mathbf{E}\left[\exp \left(g_{i j} \theta\left|\delta_{i j}\right| \xi_{i j}\right)\right]$ is guaranteed to be nonnegative and nondecreasing over $\theta \geq 0$. The following theorems are presented without proofs, as they follow exactly as their symmetric counterparts but utilize (22) and (23) as an alternative to symmetry. The requirement that $\mathbf{E}\left[\xi_{i j}\right]=0$ is still assumed to hold; this is trivially met for any known distribution by shifting $\xi_{i j}$ and transforming $M_{i j}(\theta)$ accordingly (recall $\left.M_{a \xi+b}(\theta)=\exp (b \theta) M_{\xi}(a \theta)\right)$.

Theorem 3. Given that, for all $j \in J_{i}$, random variable $\xi_{i j}$ is independent, $\mathbf{E}\left[\xi_{i j}\right]=0$, and $g_{i j}$ exists, the following probability bound of constraint violation 
holds for the ith constraint and any $\theta>0$ :

$$
\begin{aligned}
\operatorname{Pr}\left\{\sum_{j} a_{i j} x_{j}+\right. & \left.\sum_{j \in J_{i}} \xi_{i j} \hat{a}_{i j} x_{j}>b_{i}\right\} \\
& \leq \exp \left(\min _{\theta>0}\left\{-\theta \Delta_{i}+\sum_{j \in J_{i}} \Lambda_{i j}\left(g_{i j} \theta\right)\right\}\right) .
\end{aligned}
$$

(GMF8)

Theorem 4. Given that, for all $j \in J_{i}$, random variable $\xi_{i j}$ is independent, $\mathbf{E}\left[\xi_{i j}\right]=0$, and $g_{i j}$ exists, and given that it is possible to order the cumulant generating functions $\Lambda_{i j}\left(g_{i j} \theta\right)$ according to conditions $\left(\mathrm{C}_{1}\right)$, the following probability bound of constraint violation holds for the ith constraint and any $\theta>0$ :

$$
\begin{aligned}
\operatorname{Pr}\left\{\sum_{j} a_{i j} x_{j}+\right. & \left.\sum_{j \in J_{i}} \xi_{i j} \hat{a}_{i j} x_{j}>b_{i}\right\} \\
& \leq \exp \left(\min _{\theta>0}\left\{-\theta \Delta_{i}+\sum_{k \in K_{i}} \Lambda_{i k}\left(g_{i k} \theta\right)\right\}\right)
\end{aligned}
$$

where $K_{i}$ contains the first $\left[\delta_{i}^{(1)}\right]$ indices from the ordering in conditions $\left(\mathrm{C}_{1}\right)$ and $g_{i j}$ is given by equation (23). The parameter $\delta_{i}^{(1)}$ depends on $U_{i}$ and is given in Table A.3.

Theorem 5. Given that, for all $j \in J_{i}$, random variable $\xi_{i j}$ is independent, $\mathbf{E}\left[\xi_{i j}\right]=0$, and $g_{i j}$ exists, the following probability bound of constraint violation holds for the ith constraint and any $\theta>0$ :

$$
\begin{aligned}
\operatorname{Pr}\left\{\sum_{j} a_{i j} x_{j}+\sum_{j \in J_{i}} \xi_{i j} \hat{a}_{i j} x_{j}>b_{i}\right\} & \\
\leq & \exp \left(\min _{\theta>0}\left\{-\theta \Delta_{i}+\delta_{i}^{(1)} \max _{j \in J_{i}} \Lambda_{i j}\left(g_{i j} \theta\right)\right\}\right)
\end{aligned}
$$

where $g_{i j}$ is given by equation (23). The parameter $\delta_{i}^{(1)}$ depends on $U_{i}$ and is given in Table A.3.

Corollary 5.1. For ellipsoidal and interval + ellipsoidal uncertainty sets, given that $\Lambda_{i j}^{*}(\theta)=\Lambda_{i j}\left(g_{i j} \theta\right)$ satisfies condition $\left(\mathrm{C}_{2}\right)$ for all $\theta>0$ (that is, $\Lambda_{i j}(g \sqrt{\theta})$ is concave over $\theta>0$ ), the following probability bound of constraint violation 
holds for the ith constraint and any $\theta>0$ :

$$
\begin{aligned}
& \operatorname{Pr}\left\{\sum_{j} a_{i j} x_{j}+\sum_{j \in J_{i}} \xi_{i j} \hat{a}_{i j} x_{j}>b_{i}\right\} \\
& \leq \exp \left(\min _{\theta>0}\left\{-\theta \Delta_{i}+\left|J_{i}\right| \max _{j \in J_{i}} \Lambda_{i j}\left(g_{i j} \theta / \sqrt{\left|J_{i}\right|}\right)\right\}\right) .
\end{aligned}
$$

Example 4. Consider the constraint

$$
\widetilde{1} x_{1}-\widetilde{1} x_{2} \leq-\widetilde{4}
$$

where parameters $\widetilde{1},-\widetilde{1}$, and $-\widetilde{4}$ are subject to bounded uncertainty. Each parameter follows an asymmetric triangular distribution, can realize values at most $10 \%$ below or $8.75 \%$ above their expected values $1,-1$, and -4 , respectively, and can be written as:

$$
\begin{aligned}
-\widetilde{4} & =-4+0.4 \xi_{0} \\
\widetilde{1} & =1+0.1 \xi_{1} \\
-\widetilde{1} & =-1+0.1 \xi_{2},
\end{aligned}
$$

where random variable $\xi_{j} \in[-1,7 / 8], j=0,1,2$. Note that different choices for $\hat{a}_{j}$ can be made, but scaling $\xi_{j} \in[a, b] \subseteq[-1,1]$ allows full utilization of the information in Table A.4 of Appendix A. Constraint (24) can be reformulated as:

$$
\begin{aligned}
x_{1}-x_{2}+0.4 \xi_{0} x_{0}+0.1 \xi_{1} x_{1}+0.1 \xi_{2} x_{2} & \leq-4 \\
x_{0} & =-1 .
\end{aligned}
$$

The three random variables $\xi_{j}$ are modeled with an asymmetric triangular distribution supported on $[a, b]=[-1,7 / 8]$ with a mode $c=1 / 8$ (note that $\left.\mathbf{E}\left[\xi_{j}\right]=0\right)$. The moment generating function is given in Table A.4 of Appendix A; as per the table, parameter $g=-\operatorname{sgn} 1 / 8=-1$. Let us consider a probability of constraint violation of up to 0.1 ; for illustrative purposes, we will first utilize an interval + polyhedral uncertainty set, and then an interval + ellipsoidal uncertainty set. For the interval + polyhedral set, the lowest value of $\Delta_{i}$ (that is, $\Gamma$ ) will be found that guarantees a probability of 0.1 by utilizing bound (GMF8). Based on (GMF8) and as per the form in (9), we must find an optimal solution to

$$
\begin{array}{ll}
\min _{\Gamma} & \Gamma \\
\text { s.t. } & \min _{\theta>0}\{-\theta \Gamma \\
& \left.+3 \ln \left(2 \frac{\left(\frac{1}{8}+1\right) \exp \left(\frac{7}{8} g \theta\right)-\left(\frac{7}{8}+1\right) \exp \left(\frac{1}{8} g \theta\right)+\left(\frac{7}{8}-\frac{1}{8}\right) \exp (-g \theta)}{\left(\frac{7}{8}+1\right)\left(\frac{7}{8}-\frac{1}{8}\right)\left(\frac{1}{8}+1\right) \theta^{2}}\right)\right\} \\
& \Gamma \geq 0 \quad \leq \ln (0.1)
\end{array}
$$


where the logarithmic expression is the cumulant generating function of the asymmetric triangular distribution, provided in Table A.4 of Appendix A. The coefficient 3 appears because $\left|J_{i}\right|=3$, and the three cumulant generating functions in the summation of bound (GMF8) are equivalent. The argument $g \theta$ is used as per (GMF8). With $g=-1$, this simplifies to

$$
\begin{array}{ll}
\min _{\Gamma} & \Gamma \\
\text { s.t. } & \min _{\theta>0}\left\{-\theta \Gamma+3 \ln \left(\frac{64}{\theta^{2}}\left[\frac{\exp \left(-\frac{7}{8} \theta\right)}{45}-\frac{\exp \left(-\frac{1}{8} \theta\right)}{27}+\frac{2 \exp (\theta)}{135}\right]\right)\right\} \leq \ln (0.1) \\
& \Gamma \geq 0 .
\end{array}
$$

We can efficiently determine the optimal value of $\Gamma$ by using the bisection method (see Section 4 for details), which returns $\Gamma=1.423$ and yields a probability of constraint violation of 0.1 :

$$
\begin{array}{r}
\exp \left(\min _{\theta>0}\left\{-1.423 \theta+3 \ln \left(\frac{64}{\theta^{2}}\left[\frac{\exp \left(-\frac{7}{8} \theta\right)}{45}-\frac{\exp \left(-\frac{1}{8} \theta\right)}{27}+\frac{2 \exp (\theta)}{135}\right]\right)\right\}\right) \\
=\exp (-2.30)=0.10
\end{array}
$$

where $\theta^{*}=3.427$. As per Table A.2 of Appendix A, the robust counterpart of the constraint with an interval + polyhedral set is:

$$
\begin{aligned}
x_{1}-x_{2}+p_{0}+p_{1}+p_{2}+1.423 z & \leq-4 \\
z+p_{0} & \geq 0.4 \\
z+p_{1} & \geq 0.1\left|x_{1}\right| \\
z+p_{2} & \geq 0.1\left|x_{2}\right| \\
z, p_{0}, p_{1}, p_{2} & \geq 0 .
\end{aligned}
$$

For the interval + ellipsoidal set, the lowest value of $\Delta_{i}$ (that is, $\Omega$ ) will be found that guarantees a probability of 0.1 by utilizing bound (GMF10'). The maximization operator is dropped because the cumulant generation functions of $\xi_{j}$ are equivalent. Based on $\left(\mathrm{GMF}^{\prime} 0^{\prime}\right)$ and as per the form in (9), we must find an optimal solution to

$$
\begin{array}{ll}
\min _{\Omega} & \Omega \\
\text { s.t. } & \min _{\theta>0}\{-\theta \Omega \\
& \left.+3 \ln \left(2 \frac{\left(\frac{1}{8}+1\right) \exp \left(\frac{7}{8} \frac{g \theta}{\sqrt{3}}\right)-\left(\frac{7}{8}+1\right) \exp \left(\frac{1}{8} \frac{g \theta}{\sqrt{3}}\right)+\left(\frac{7}{8}-\frac{1}{8}\right) \exp \left(-\frac{g \theta}{\sqrt{3}}\right)}{\left(\frac{7}{8}+1\right)\left(\frac{7}{8}-\frac{1}{8}\right)\left(\frac{1}{8}+1\right) \theta^{2} / 3}\right)\right\} \\
& \Omega \geq 0 \quad \leq \ln (0.1) \quad
\end{array}
$$

where the logarithmic expression is the cumulant generating function of the asymmetric triangular distribution, provided in Table A.4 of Appendix A. The 
coefficient 3 appears because $\left|J_{i}\right|=3$, and maximization operator is dropped because the three cumulant generating functions are equivalent. The argument $g \theta / \sqrt{3}$ is used as per $\left(\mathrm{GMF}^{\prime} 0^{\prime}\right)$. With $g=-1$, this simplifies to

$$
\begin{array}{ll}
\min _{\Omega} & \Omega \\
\text { s.t. } & \min _{\theta>0}\{-\theta \Omega \\
& \left.+3 \ln \left(\frac{64}{\theta^{2}}\left[\frac{\exp \left(-\frac{7}{8 \sqrt{3}} \theta\right)}{15}-\frac{\exp \left(-\frac{1}{8 \sqrt{3}} \theta\right)}{9}+\frac{2 \exp \left(\frac{1}{\sqrt{3}} \theta\right)}{45}\right]\right)\right\} \leq \ln (0.1) \\
& \Omega \geq 0 .
\end{array}
$$

We can efficiently determine the optimal value of $\Omega$ by using the bisection method (see Section 4 for details), which returns $\Omega=0.822$ and yields a probability of constraint violation of 0.1 :

$$
\begin{gathered}
\exp \left(\min _{\theta>0}\left\{-0.822 \theta+3 \ln \left(\frac{64}{\theta^{2}}\left[\frac{\exp \left(-\frac{7}{8 \sqrt{3}} \theta\right)}{15}-\frac{\exp \left(-\frac{1}{8 \sqrt{3}} \theta\right)}{9}+\frac{2 \exp \left(\frac{1}{\sqrt{3}} \theta\right)}{45}\right]\right)\right\}\right) \\
=\exp (-2.30)=0.10,
\end{gathered}
$$

where $\theta^{*}=5.940$. As per Table A.2 of Appendix A, the robust counterpart of the constraint with an interval + ellipsoidal set is:

$$
\begin{aligned}
x_{1}-x_{2}+0.4 \mid-1 & -z_{0}|+0.1| x_{1}-z_{1} \mid \\
& +0.1\left|x_{2}-z_{2}\right|+0.822 \sqrt{0.16 z_{0}^{2}+0.01 z_{1}^{2}+0.01 z_{2}^{2}} \leq-4 .
\end{aligned}
$$

Note that (GMF8) extends (KBP1); (GMF9) extends (GMF6); (GMF10) extends (GMF7); and (GMF10') extends (GMF7').

The discussion in Section 3 included situations where, based on the mathematical structure of the bounds, it can be seen that certain bounds will always be better than other bounds. A more comprehensive overview is given in Table 7 , where it is apparent that the new proposed a priori bounds outperform almost all existing ones.

\section{Implementation}

As discussed in Part I (Guzman et al., 2016a), while the a priori bounds provide an upper bound on the probability of constraint violation for a specified $\Delta_{i}$, the robust counterpart formulation requires a value of $\Delta_{i}$ (ideally, the lowest possible value) which will guarantee a specified $\epsilon_{i}^{\text {prio }}$. As seen in Section 2, we must find an optimal solution to

$$
\begin{array}{ll}
\min _{\Delta_{i}} & \Delta_{i} \\
\text { s.t. } & B\left(\Delta_{i}\right) \leq \epsilon_{i}^{\text {prio }} \\
& \Delta_{i} \geq 0
\end{array}
$$


where $B\left(\Delta_{i}\right)$ is an a priori bound expression. The new bounds presented in this paper (as well as some existing bounds) follow a particular form and include auxiliary variable $\theta$ :

$$
\begin{array}{ll}
\min _{\Delta_{i}} & \Delta_{i} \\
\text { s.t. } & \min _{\theta>0}\left\{-\theta \Delta_{i}+b(\theta)\right\} \leq \ln \epsilon_{i}^{\text {prio }} \\
& \Delta_{i} \geq 0
\end{array}
$$

where $b(\theta)$ is derived from the cumulant generating functions of the distributions of random variables $\xi_{i j}$. Note that $b(\theta)$ is a convex function because convexity is a property of all cumulant generating functions (e.g., see Boyd and Vandenberghe, 2004). In the context of problem (28), the inner minimization operator can be removed due to its location in the inequality:

$$
\begin{array}{cl}
\min _{\Delta_{i}, \theta} & \Delta_{i} \\
\text { s.t. } & -\theta \Delta_{i}+b(\theta) \leq \ln \epsilon_{i}^{\text {prio }} \\
& \Delta_{i} \geq 0 \\
& \theta>0 .
\end{array}
$$

Problem (29) is nonconvex due to the bilinear term $\theta \Delta_{i}$. If the nonconvex constraint is active in an optimal solution (as is always the case with the new bounds of this work, but not for, say, bound (GMF3)), the constraint can be eliminated by rearranging it to $\Delta_{i} \geq \theta^{-1}\left(b(\theta)-\ln \epsilon_{i}^{\text {prio }}\right)$ and substituting it into the objective function:

$$
\Delta_{i}=\min _{\theta>0}\left\{\frac{b(\theta)-\ln \epsilon_{i}^{\text {prio }}}{\theta}\right\} .
$$

It may be difficult to computationally determine the extrema of the function in the minimization, as $\theta^{*}$ may lie in a nearly flat basin given bounded probability distributions due to the fact that their cumulant generating functions (contained in $b(\theta)$ ) are near-linear when away from $\theta=0$. For example, if $b(\theta)=\ln (\sinh (\theta) / \theta)$ (that is, composed from the cumulant generating function of a single uniform distribution), and $\epsilon_{i}^{\text {prio }}=0.01$, the objective function of (30) is equal to 1 at $\theta=50$ and 0.9954 at $\theta=500$.

An alternative to utilizing one of (9), (28)-(30), is to observe that since $\epsilon_{i}^{\text {prio }}$ changes monotonically with $\Delta_{i}$ in all of the bounds, an appropriate $\Delta_{i}$ can be found via the bisection method over $\Delta_{i}$, that is, by selecting $\Delta_{i}$, checking the value returned by a bound expression, and adjusting the value of $\Delta_{i}$ if necessary. Note that this may be computationally intensive for bounds presented in this work, as they contain an inner minimization (albeit a convex one) over $\theta>0$. However, the following proposition allows the inner minimization to be efficiently handled. 
Proposition 1. Given the nonlinear optimization problem (NLP)

$$
\min _{x}\left\{-c x+f(x): x \geq x^{L}\right\}
$$

where $c>0, f(x)$ is a $\mathcal{C}^{1}$-continuous convex function over $x \geq x^{L}$ with $f\left(x^{L}\right)=c x^{L}$ and $0 \leq d f(x) /\left.d x\right|_{x^{L}}<c$, and $x^{*}$ is an optimal solution, then

(i) $x^{*}>x^{L}$,

(ii) $x^{*}$ is nondecreasing as c increases.

Proof. See (Guzman et al., 2016a).

If Proposition 1 applies to the inner minimization, then the constraint $\theta>0$ is redundant and the inner minimization can be solved via simple algorithms such as a golden section search. Note that the condition $0 \leq d f(x) /\left.d x\right|_{x^{L}}<c$ will always hold for $f(x)=b(\theta)$ and $c=\Delta_{i}>0 ; d b(\theta) /\left.d \theta\right|_{0}=0$ because $b(\theta)$ is composed of cumulant generating functions of distributions with a mean of zero, and the first derivative of a cumulant generating function at $\theta=0$ is equal to the mean of the distribution (e.g., see Pistone and Wynn, 1999). Computational cost can be reduced further by considering that first-order optimality conditions can bypass the minimization completely by providing $\Delta_{i}$ as a function of $\theta^{*}$. Thus, to seek $\epsilon_{i}^{\text {prio }}$, a bisection algorithm would select a value of $\theta^{*}$, calculate a corresponding value of $\Delta_{i}$ from first-order optimality conditions, and then calculate the probability of constraint violation using the bound and compare it with $\epsilon_{i}^{\text {prio }}$. This approach is summarized in Algorithm 1. Setting the first derivative of bound (GMF6) to zero and solving for $\Delta_{i}$ :

$$
\Delta_{i}\left(\theta^{*}\right)=\left.\sum_{k \in K_{i}} \frac{d \Lambda_{i k}(\theta)}{d \theta}\right|_{\theta^{*}} .
$$

Function $\Delta_{i}\left(\theta^{*}\right)$ for bound (KBP1) is similar to (31), with index $j$ and set $J_{i}$ replacing $k$ and $K_{i}$, respectively. For bound (GMF9),

$$
\Delta_{i}\left(\theta^{*}\right)=\left.\sum_{k \in K_{i}} g_{i k} \frac{d \Lambda_{i k}(\theta)}{d \theta}\right|_{g_{i k} \theta^{*}} .
$$

Function $\Delta_{i}\left(\theta^{*}\right)$ for bound (GMF8) is similar to (32), with index $j$ and set $J_{i}$ replacing $k$ and $K_{i}$, respectively. 


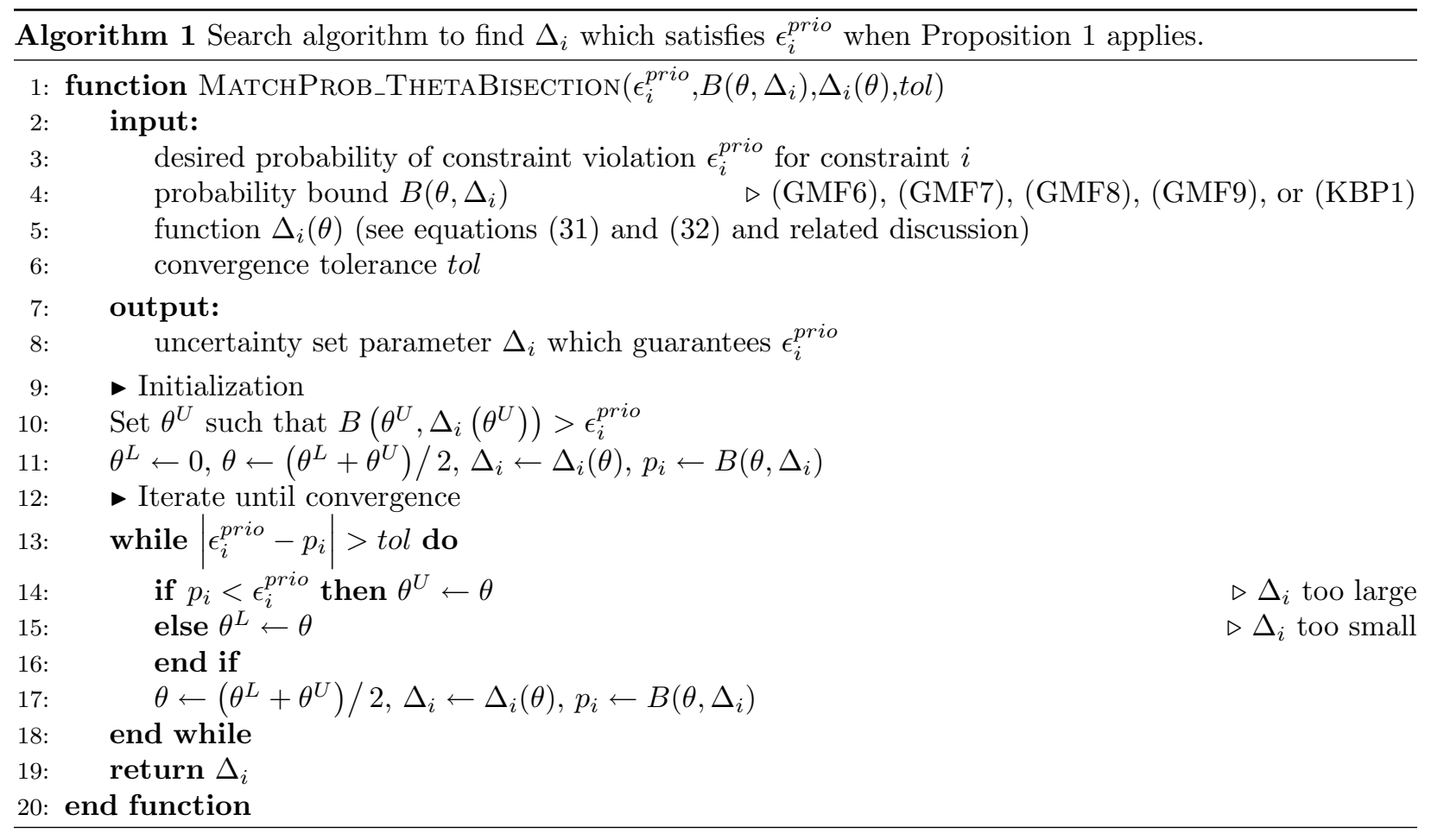


Proposition 1 does not always fully characterize bounds which utilize the maximization operator, such as (GMF7), as they are not necessarily $\mathcal{C}^{1}$-continuous. If one of the cumulant generating functions can replace the maximization operator because it is always greater than or equal to the rest, then Proposition 1 still applies and first-order optimality conditions can be used as in the preceding section. Otherwise, the bisection method can still be applied, but in a manner different from the preceding section. Note that since the expected values of the random variables are assumed to be zero, the first derivative of all participating moment generating functions (and their $g_{i j}$-parameterized variants in the asymmetric case) at $\theta=0$ is 0 ; thus, it still holds that $\theta^{*}>0$ for $\Delta_{i}>0$ and an algorithm such as a golden section search can still be applied.

To obtain an algorithm solely reliant on the bisection method, consider the following reformulation on the inner minimization of bound (GMF7):

$$
\begin{array}{ll}
\min _{\theta, w} & -\theta \Delta_{i}+\delta_{i}^{(1)} w \\
\text { s.t. } & w \geq \Lambda_{i j}(\theta) \quad \forall j \in J_{i} .
\end{array}
$$

The unconstrained minimization is reformulated into a constrained NLP. At an optimal solution $\left(\theta^{*}, w^{*}\right)$,

$$
w^{*}=\max _{j \in J_{i}} \Lambda_{i j}\left(\theta^{*}\right) .
$$

Slater's condition qualifies the use of KKT conditions and implies that strong duality holds (Floudas, 1995; Slater, 1950). At an optimal solution $\left(\theta^{*}, w^{*}, \alpha^{*}\right)$,

$$
\begin{array}{ll}
\Delta_{i}=\left.\sum_{j \in J_{i}} \alpha_{j}^{*} \frac{d \Lambda_{i j}(\theta)}{d \theta}\right|_{\theta=\theta^{*}} & \\
\delta_{i}^{(1)}=\sum_{j \in J_{i}} \alpha_{j}^{*} & \forall j \in J_{i} \\
\alpha_{j}^{*}\left(\Lambda_{i j}\left(\theta^{*}\right)-w^{*}\right)=0 & \forall j \in J_{i} \\
\alpha_{j}^{*} \geq 0 &
\end{array}
$$

where variables $\alpha_{j}$ are Lagrange multipliers. We define the following set:

$$
J_{i}^{\alpha}(\theta):=\left\{j: j \in J_{i}, \Lambda_{i j}(\theta)=\max _{k \in J_{i}} \Lambda_{i k}(\theta)\right\}
$$

which, due to (34) and (37), defines indices $j$ for which $\alpha_{j}^{*}$ may be nonzero. In other words, the only $j$ for which $\alpha_{j}$ can be nonzero are those which correspond to $\Lambda_{i j}\left(\theta^{*}\right)=\max _{k \in J_{i}} \Lambda_{i k}\left(\theta^{*}\right)$. Equation (35) can be rewritten as

$$
\Delta_{i}=\sum_{j \in J_{i}^{\alpha}} \alpha_{j} \frac{d \Lambda_{i j}(\theta)}{d \theta}
$$

Say a value of $\theta^{*}$ is selected. In the preceding section, there existed a corresponding value of $\Delta_{i}$ such that $\theta^{*}$ was an optimal solution. If $\left|J_{i}^{\alpha}\left(\theta^{*}\right)\right|=1$ 
(that is, one cumulant generating function is greater than the rest at $\theta^{*}$ ), then (34) and the KKT conditions (35)-(38) fully specify $\alpha^{*}$ and $\Delta_{i}$. However, if $\left|J_{i}^{\alpha}\left(\theta^{*}\right)\right|>1$ (that is, multiple cumulant generating functions intersecting at $\left.\theta^{*}\right)$, there exist multiple combinations of $\alpha^{*}$ and $\Delta_{i}$ which can satisfy the KKT conditions for the selected $\theta^{*}$. One can examine equations (36), (38), and (40) and obtain a range of values that $\Delta_{i}$ can take while still permitting $\theta^{*}$ as an optimal solution:

$$
\begin{aligned}
& \Delta_{i}^{L}\left(\theta^{*}\right)=\delta_{i}^{(1)} \min _{j \in J_{i}^{\alpha}\left(\theta^{*}\right)}\left\{\left.\frac{d \Lambda_{i j}(\theta)}{d \theta}\right|_{\theta=\theta^{*}}\right\} \\
& \Delta_{i}^{U}\left(\theta^{*}\right)=\delta_{i}^{(1)} \max _{j \in J_{i}^{\alpha}\left(\theta^{*}\right)}\left\{\left.\frac{d \Lambda_{i j}(\theta)}{d \theta}\right|_{\theta=\theta^{*}}\right\} .
\end{aligned}
$$

Thus, implementation of a bisection method would differ from the preceding section by calculating $\Delta_{i}^{L}\left(\theta^{*}\right)$ and $\Delta_{i}^{U}\left(\theta^{*}\right)$ instead of a single $\Delta_{i}\left(\theta^{*}\right)$. If $\Delta_{i}^{L}\left(\theta^{*}\right)=\Delta_{i}^{U}\left(\theta^{*}\right)$, the algorithm would proceed as before. If $\Delta_{i}^{L}\left(\theta^{*}\right) \neq \Delta_{i}^{U}\left(\theta^{*}\right)$, then a secondary check must be made. If $\epsilon_{i}^{\text {prio }} \in\left[\epsilon_{i}\left(\theta^{*}, \Delta_{i}^{U}\right), \epsilon_{i}\left(\theta^{*}, \Delta_{i}^{L}\right)\right]$, a $\Delta_{i}$ corresponding to $\epsilon_{i}^{\text {prio }}$ lies in $\left[\Delta_{i}^{L}\left(\theta^{*}\right), \Delta_{i}^{U}\left(\theta^{*}\right)\right]$ and the bisection algorithm should thereafter seek $\epsilon_{i}^{\text {prio }}$ by searching $\Delta_{i}$ at fixed $\theta^{*}$. Otherwise, the algorithm would select $\epsilon_{i}\left(\theta^{*}, \Delta_{i}^{L}\right)$ or $\epsilon_{i}\left(\theta^{*}, \Delta_{i}^{U}\right)$, whichever is closer to $\epsilon_{i}^{\text {prio }}$, at that iteration and proceed as before. This approach is summarized in Algorithm 2.

Equations (41) and (42) apply to bound (GMF7). A similar analysis can be made for the other bounds with the maximization operator. For bound (GMF $\left.7^{\prime}\right)$,

$$
\begin{aligned}
& \Delta_{i}^{L}\left(\theta^{*}\right)=\sqrt{\left|J_{i}\right|} \min _{j \in J_{i}^{\alpha}\left(\theta^{*}\right)}\left\{\left.\frac{d \Lambda_{i j}(\theta)}{d \theta}\right|_{\theta=\theta^{*} / \sqrt{\left|J_{i}\right|}}\right\} \\
& \Delta_{i}^{U}\left(\theta^{*}\right)=\sqrt{\left|J_{i}\right|} \max _{j \in J_{i}^{\alpha}\left(\theta^{*}\right)}\left\{\left.\frac{d \Lambda_{i j}(\theta)}{d \theta}\right|_{\theta=\theta^{*} / \sqrt{\left|J_{i}\right|}}\right\} .
\end{aligned}
$$

For bound (GMF10),

$$
\begin{aligned}
& \Delta_{i}^{L}\left(\theta^{*}\right)=\delta_{i}^{(1)} \min _{j \in J_{i}^{\alpha}\left(\theta^{*}\right)}\left\{\left.g_{i j} \frac{d \Lambda_{i j}(\theta)}{d \theta}\right|_{\theta=g_{i j} \theta^{*}}\right\} \\
& \Delta_{i}^{U}\left(\theta^{*}\right)=\delta_{i}^{(1)} \max _{j \in J_{i}^{\alpha}\left(\theta^{*}\right)}\left\{\left.g_{i j} \frac{d \Lambda_{i j}(\theta)}{d \theta}\right|_{\theta=g_{i j} \theta^{*}}\right\} .
\end{aligned}
$$

For bound $\left(\mathrm{GMF} 10^{\prime}\right)$,

$$
\begin{aligned}
& \Delta_{i}^{L}\left(\theta^{*}\right)=\sqrt{\left|J_{i}\right|} \min _{j \in J_{i}^{\alpha}\left(\theta^{*}\right)}\left\{\left.g_{i j} \frac{d \Lambda_{i j}(\theta)}{d \theta}\right|_{\theta=g_{i j} \theta^{*} / \sqrt{\left|J_{i}\right|}}\right\} \\
& \Delta_{i}^{U}\left(\theta^{*}\right)=\sqrt{\left|J_{i}\right|} \max _{j \in J_{i}^{\alpha}\left(\theta^{*}\right)}\left\{\left.g_{i j} \frac{d \Lambda_{i j}(\theta)}{d \theta}\right|_{\theta=g_{i j} \theta^{*} / \sqrt{\left|J_{i}\right|}}\right\} .
\end{aligned}
$$




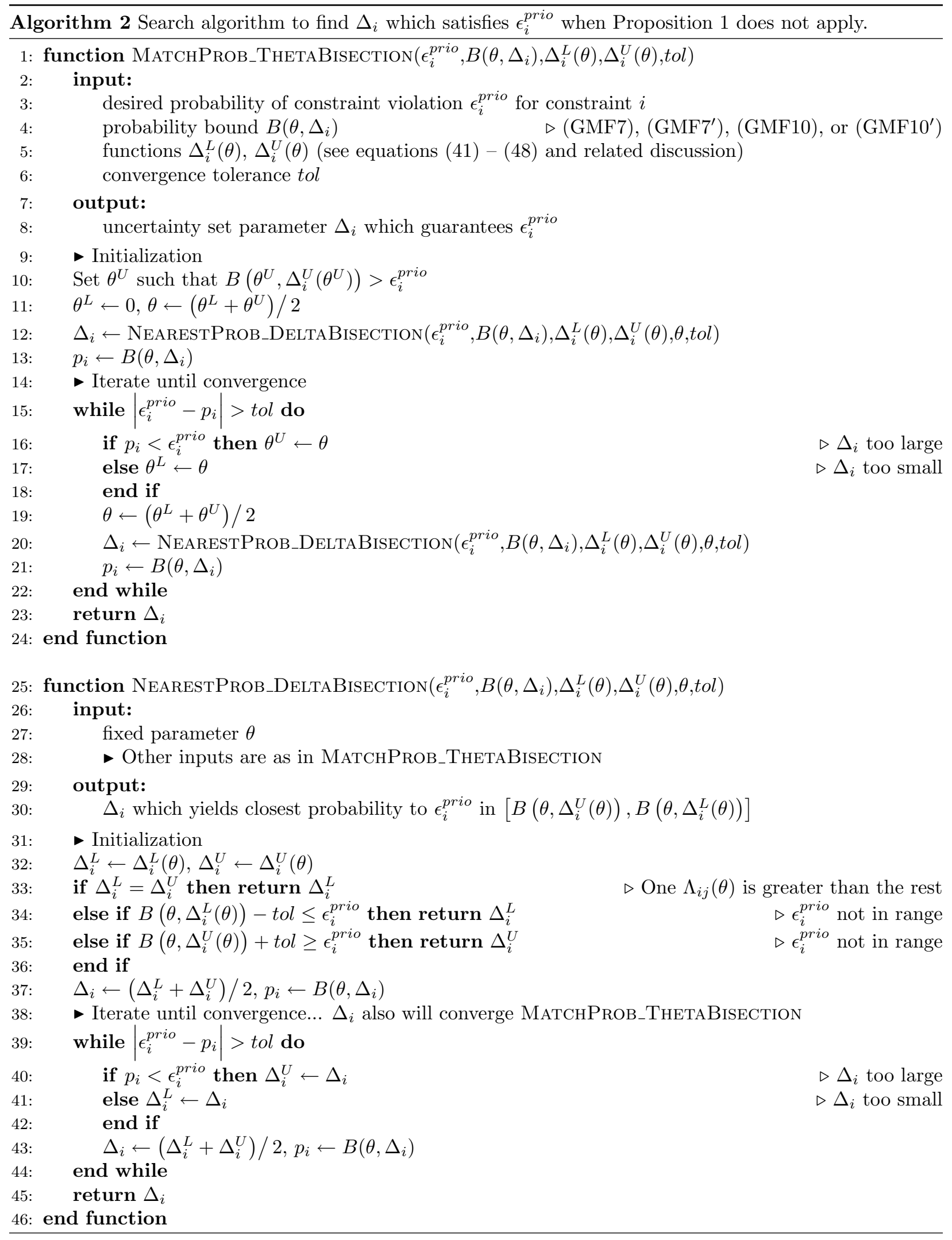




\section{Comparisons of a priori bounds}

This section compares the existing and new a priori bounds computationally with two example problems, a linear optimization problem (LP) and a mixedinteger linear optimization problem (MILP), to demonstrate the improvements made in this work. A concise summary of the best bounds for each uncertainty type based on each bound's mathematical structure is found in Table 7. A summary of the best performing bounds in the following case studies is shown in Table 3 .

\subsection{Example MILP}

As in Part I (Guzman et al., 2016a), a modified version of Example 7.1 in Li et al. (2011) is used as an example MILP for the testing of the proposed bounds. The nominal MILP is

$$
\begin{array}{cl}
\max _{x, y} & \widetilde{3} x_{1}+\widetilde{2} x_{2}-\widetilde{10} y_{1}-5 y_{2} \\
\text { s.t. } & \widetilde{1} x_{1}+\widetilde{1} x_{2} \leq \widetilde{20} \\
& \widetilde{1} x_{1}+\widetilde{2} x_{2} \leq \widetilde{12} \\
& x_{1}-20 y_{1} \leq 0 \\
& x_{2}-20 y_{2} \leq 0 \\
& \widetilde{1} x_{1}-\widetilde{1} x_{2} \leq-\widetilde{4} \\
& 0 \leq x_{1} \leq 10 \\
& 0 \leq x_{2} \leq 10 \\
& y_{1}, y_{2} \in\{0,1\} .
\end{array}
$$

Evaluating the robust counterpart of this MILP using $\Delta_{i}$ values calculated from the a priori bounds will demonstrate the tightness of the bounds. In this sense, tightness refers to the ability to have a smaller $\Delta_{i}$ value, and thus uncertainty set, for the same probability of constraint violation.

Consistent with Part I, bounded uncertainty exists in right-hand side, lefthand side, and objective function parameters. Here, all parameters denoted with a tilde (12 in total) with be attributed a full probability distribution. Each uncertain constraint $i$ has three uncertain parameters $\left(\left|J_{i}\right|=3\right)$. In each case study, the same three probability distributions will be applied to $\xi_{i j}, j \in J_{i}$, in each uncertain constraint $i$, yielding the same values of $\Delta_{i}$ with a given bound and uncertainty set type. Each uncertain parameter $\tilde{a}_{i j}$ can realize values that are, at most, $10 \%$ away from the nominal parameter values; thus, $\hat{a}_{i j}=0.1 a_{i j}$.

\subsubsection{Case Study 1: Applications of the Raised Cosine Distribution to Param- eters with Unknown Distributions}

It is often the case that the probability distribution of uncertainty parameters is unknown, and the use of the a priori bounds derived in Part I can be convenient and effective (Guzman et al., 2016a). Yet, it is often a very reasonable, conservative approximation to use the uniform distribution function as 
a distribution for the uncertain data. In the context of the bounds presented in this work, utilizing an overestimator to a distribution's cumulant generating function will yield a more conservative solution than utilizing the distribution's precise cumulant generating function. The cumulant generating function of a uniform distribution supported on $[-1,1]$ has been previously shown to overestimate the cumulant generating function of any symmetric and unimodal (with respect to 0) distribution supported on $[-1,1]$ (Nemirovski and Shapiro, 2007). It can also overestimate certain asymmetric distributions with probability density concentrated about the mean, such as a triangular distribution (see Table A.4 of Appendix A). We note that the use of the conservative uniform assumption greatly benefits the overall reduction of conservatism in robust optimization when using probabilistic bounds. A uniform distribution would not conservatively approximate a distribution which yields higher probabilities near the bounds of the support rather than near the mean (say, exhibiting an inverted bell-shaped distribution). However, observed values of an uncertain parameter are often concentrated around the mean and exhibit a bell-shaped distribution. For example, if a parameter represents a price, it may be reasonable to assume, perhaps based on historical data, that the price hovers near the expected value with diminishing probability that the price will be perturbed farther from the expected value. A uniform distribution would then conservatively approximate this situation for robust optimization by decreasing probability density around the expected value and increasing the probability of (undesired) realizations at extreme values.

To study this interplay between a uniform distribution and bell-shaped distributions, one can parameterize the raised cosine distribution, which is bounded and bell-shaped. The probability density function of the parameterized raised cosine distribution with $\mathbf{E}\left[\xi_{i j}\right]=0$ and $\xi_{i j} \in[-1,1]$ is

$$
p d f(x)=\frac{1}{2}(1+d \cos (\pi x)), \quad x \in[-1,1],
$$

where the proposed parameter $d$ is bounded in the interval $[0,1]$. When the $d=1$, the distribution is equivalent to the traditional raised cosine distribution. As $d$ decreases, the distribution loses its bell shape and probability density concentrated at the bounds of the support increases, until the distribution is equivalent to a uniform distribution with $d=0$. This behavior is graphically shown in Figure 1.

Thus, if a distribution is expected to be bell-shaped, an approximation for an unknown distribution can be made heuristically at varying degrees by simply decreasing the value of $d$. The most conservative approximation of this shape would be the uniform distribution; as Figures 2 and 3 show, even the uniform approximation with bound (GMF6) will often produce large improvements on values of $\Delta_{i}$ and the resulting objective function values of the robust counterpart when compared to bound (GMF2). For example, at a $50 \%$ probability of constraint violation in Figure 3, bound (GMF2) essentially gives the worst case value of the objective function, 3.84. Bound (GMF6) with the uniform distribution, on the other hand, gives an objective function value of 4.91. This is an 


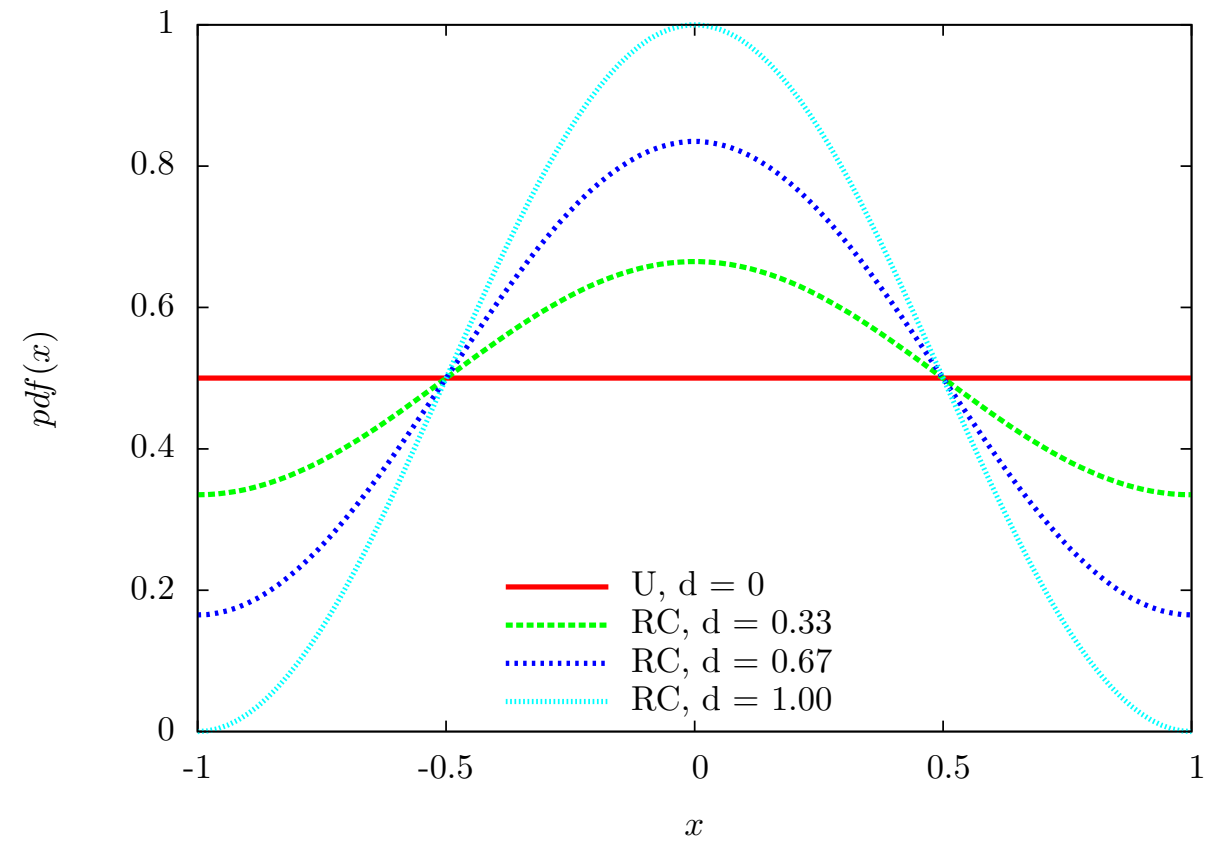

Figure 1: Probability density function for the raised cosine (RC) distribution with the bell shape gradually decreased to the uniform (U) distribution by varying the newly proposed parameter $d$ from 1 to 0 .

increase of nearly $28 \%$ simply through a conservative estimation of a uniform distribution. As the estimation become less conservative, as the various raised cosine distributions demonstrate, the delta values decrease and the objective function values increase.

Because the new a priori bounds utilize cumulant generating functions, the attribution of a uniform distribution can also provide more conservative results than some asymmetric distributions. For example, one can observe that the cumulant generating function of asymmetric triangular distributions of the form given in Table A.4 (and their $g$-parameterized variants) will always be less than or equal to that of the uniform distribution, and thus will always provide equivalent or less conservative results. This is in spite of the fact that much of the probability density is concentrated on one side and away from the expected value.

\subsubsection{Case Study 2: Multiple Parameters with Uniform Probability Distribu- tions}

Case Study 2 exemplifies the situation in which all uncertain parameters are either known to have uniform distributions, or have been approximated by this distribution as suggested in Section 5.1.1. Figures 4 through 9 demonstrate 


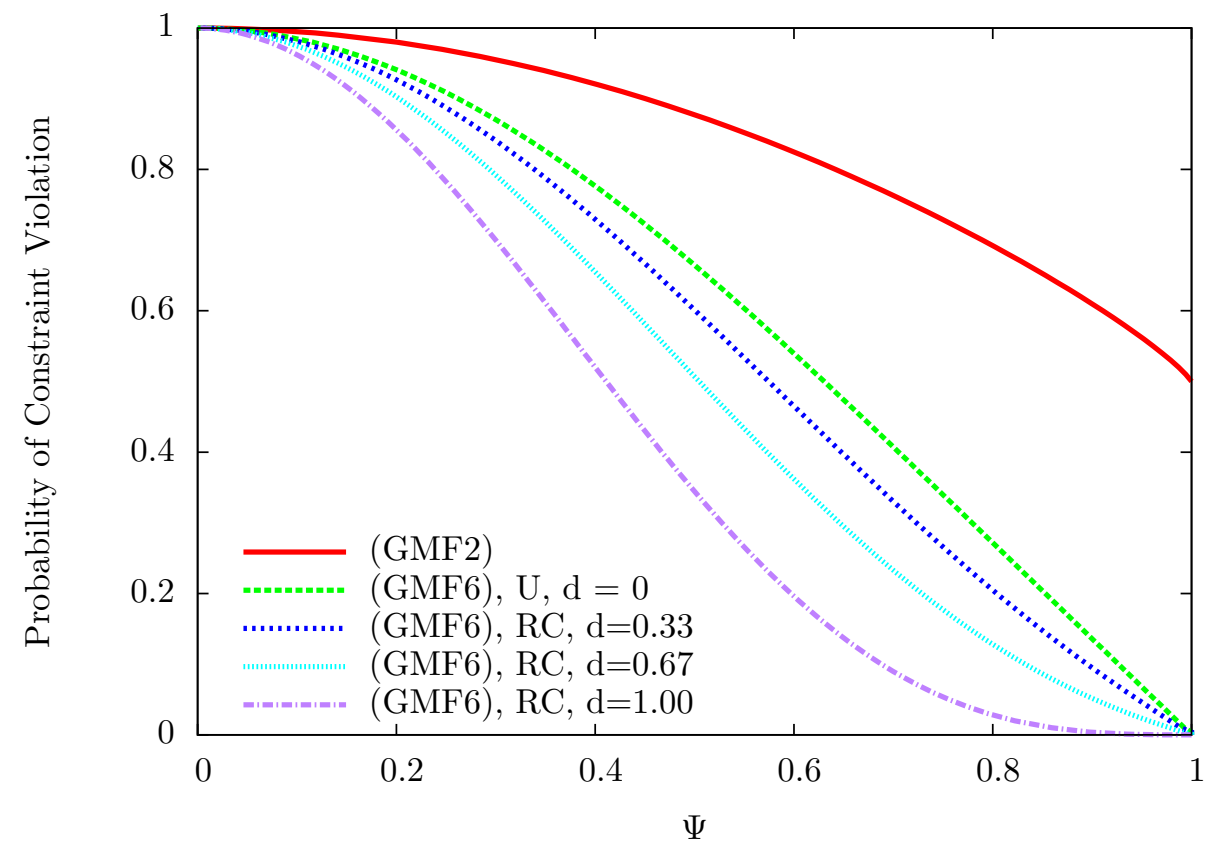

Figure 2: Probability of constraint violation versus $\Psi$ for the box uncertainty set, $\left|J_{i}\right|$, using the raised cosine (RC) distribution with parameter $d$ varied between 1 and 0 , at which point it is the uniform (U) distribution. Marked improvement in $\Psi$ is seen by approximating the unknown distribution with a uniform distribution. 


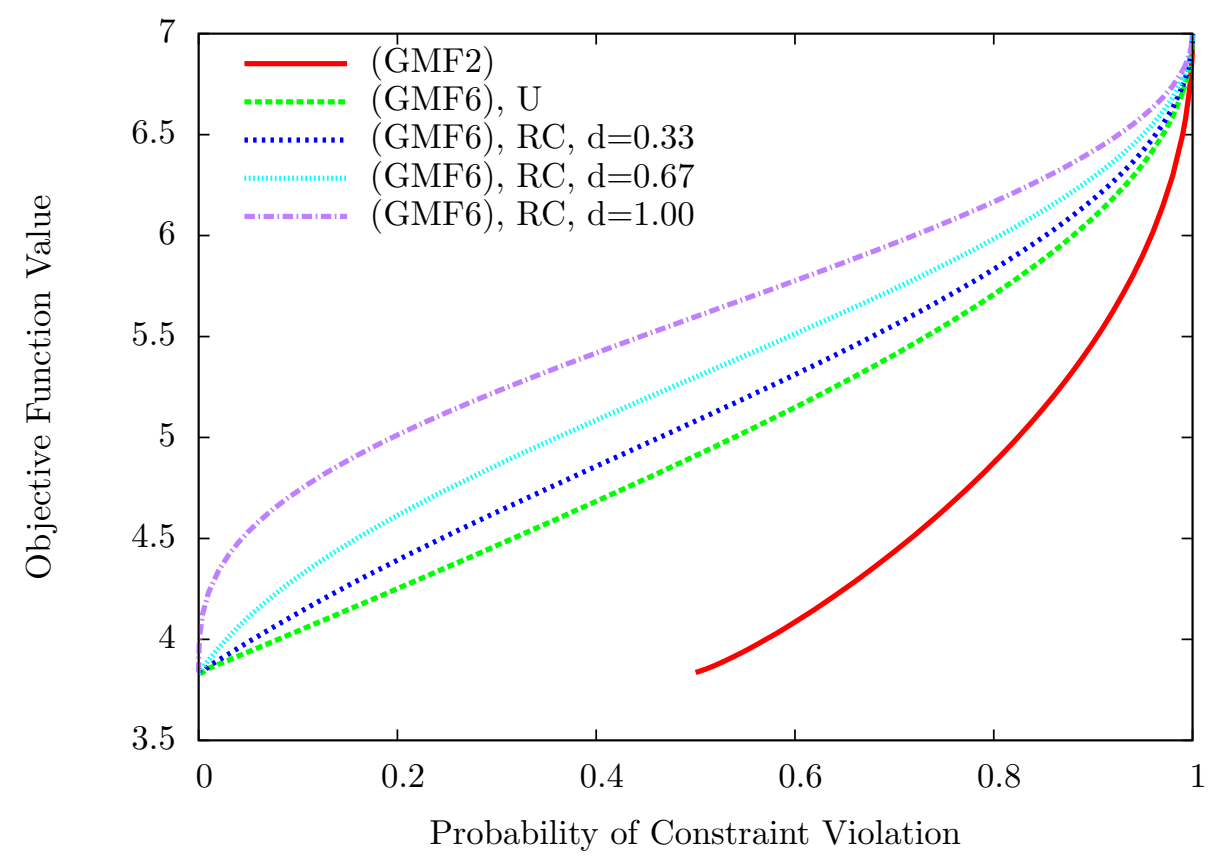

Figure 3: Objective function values from the example MILP using distribution-independent bound (GMF2) and distribution-dependent bound (GMF6). Parameters were attributed uniform $(\mathrm{U})$ or parameterized raised cosine $(\mathrm{RC})$ distributions, and a box uncertainty set was used for all three uncertain constraints and the uncertain objective function, each with $\left|J_{i}\right|=3$. 


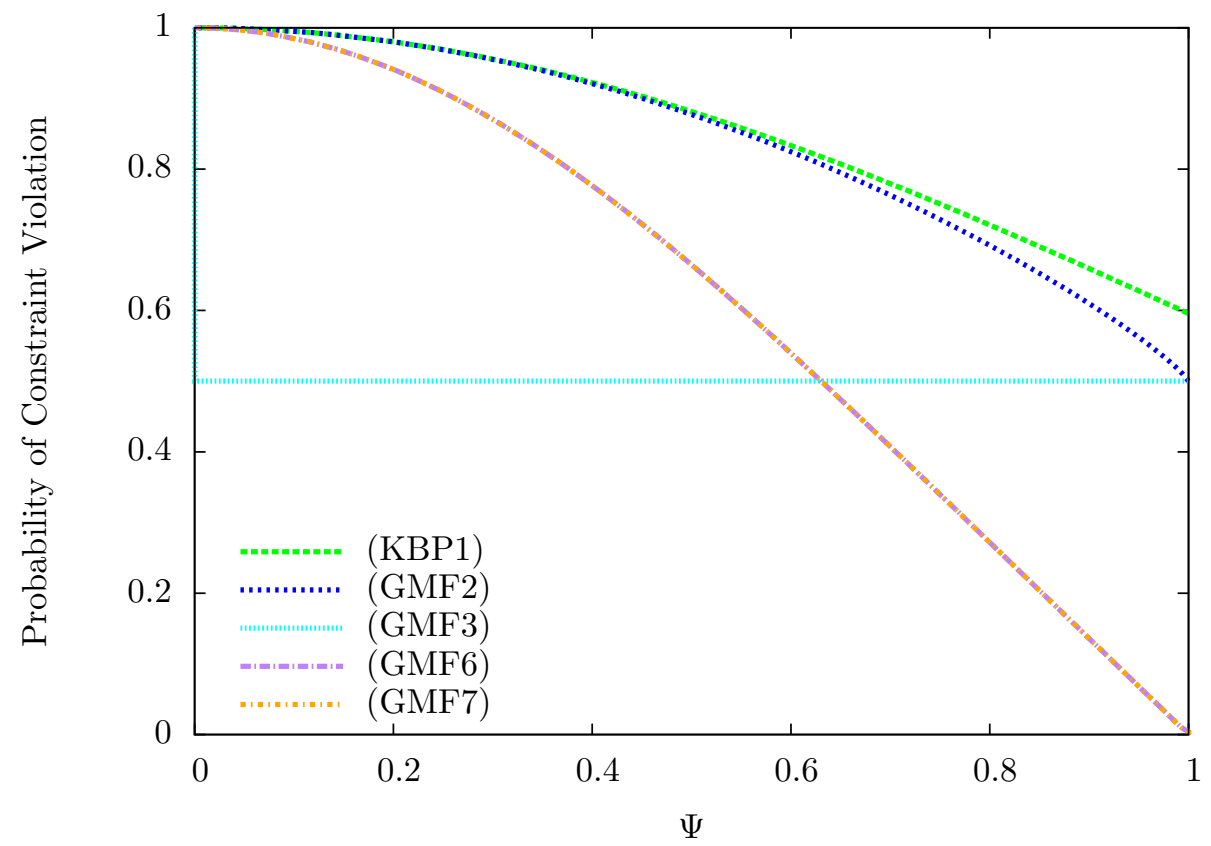

Figure 4: Probability of constraint violation versus $\Psi$ for the box uncertainty set using $a$ priori probabilities of constraint violation for a case with uniform probability distributions, $\left|J_{i}\right|=3$.

the performance of bounds in this case for the example MILP with $\left|J_{i}\right|=3$. Bounds (GMF2), (GMF2'), and (GMF3) are included due to their position as the strongest performing distribution-independent bounds. As Table 7 and Figures 4 through 9 show, there is no single bound which will be tightest at every probability of constraint violation. However, general trends certainly hold.

In all uncertainty sets, the bound (GMF3) remains dominant at higher probabilities of constraint violation. This dominance loosens at lower probabilities, and the bounds that outperform (GMF3) differ by each uncertainty set. For the box uncertainty set, it is clearly seen from Figures 4 and 5 that (GMF6) and (GMF7) behave identically, and outperform (GMF3) at probabilities of constraint violation less than $50 \%$ for $\left|J_{i}\right|=3$. Bounds (KBP1) and (GMF2) are always more conservative in this case. The tight performance of bound (GMF7'), which features ellipsoidal-specific improvements to bound (GMF7), is evident in Figures 6 and 7 at probabilities of constraint violation below $50 \%$, exhibiting great reductions in $\Omega$ at very low $\epsilon_{i}^{\text {prio }}$. Surprisingly, as Figures 4 through 7 show, distribution-independent bounds (GMF2) and (GMF2') are able to out-perform the previously-existing distribution-dependent bound (KBP1) in box and interval + ellipsoidal sets, respectively, though they are looser than 


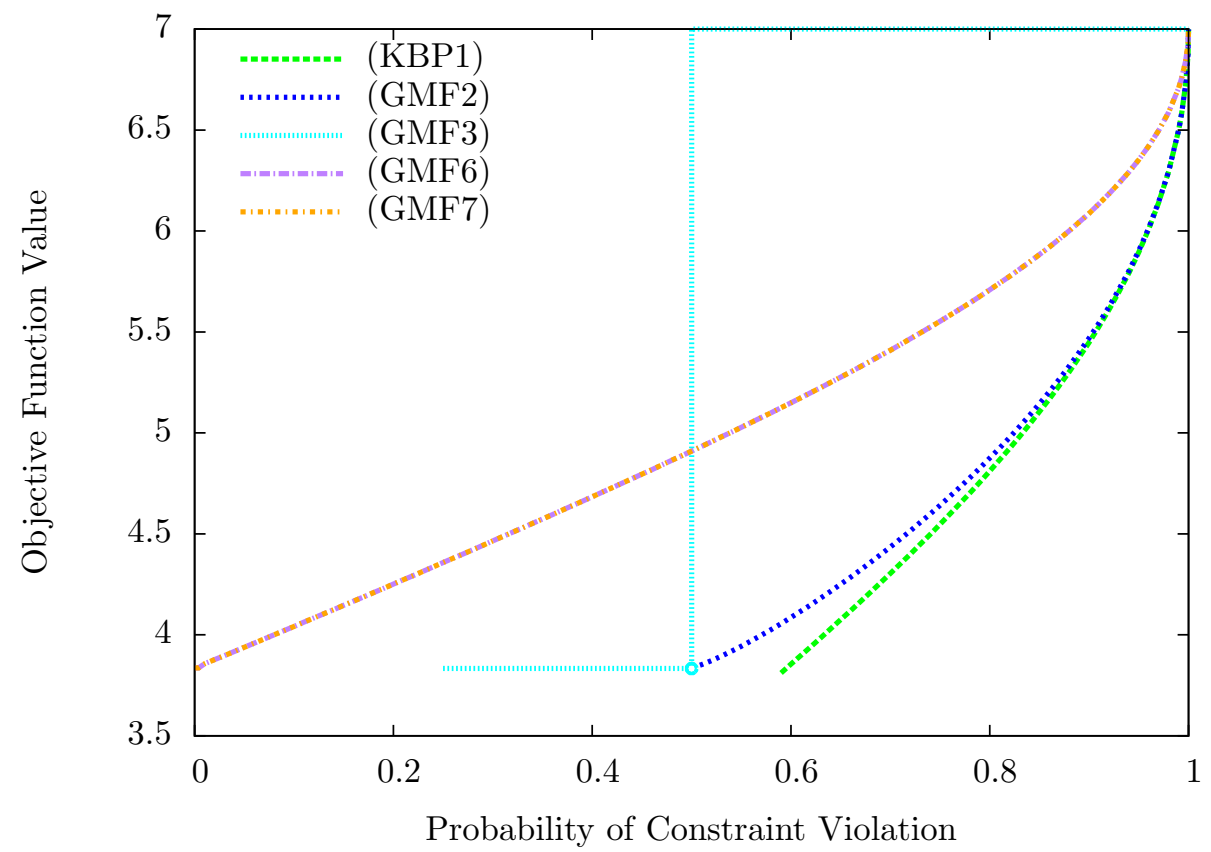

Figure 5: Objective function values from the example MILP at varying probabilities of constraint violation for the box uncertainty set, using a priori bounds for a case with uniform probability distributions for all parameters, $\left|J_{i}\right|=3$. 


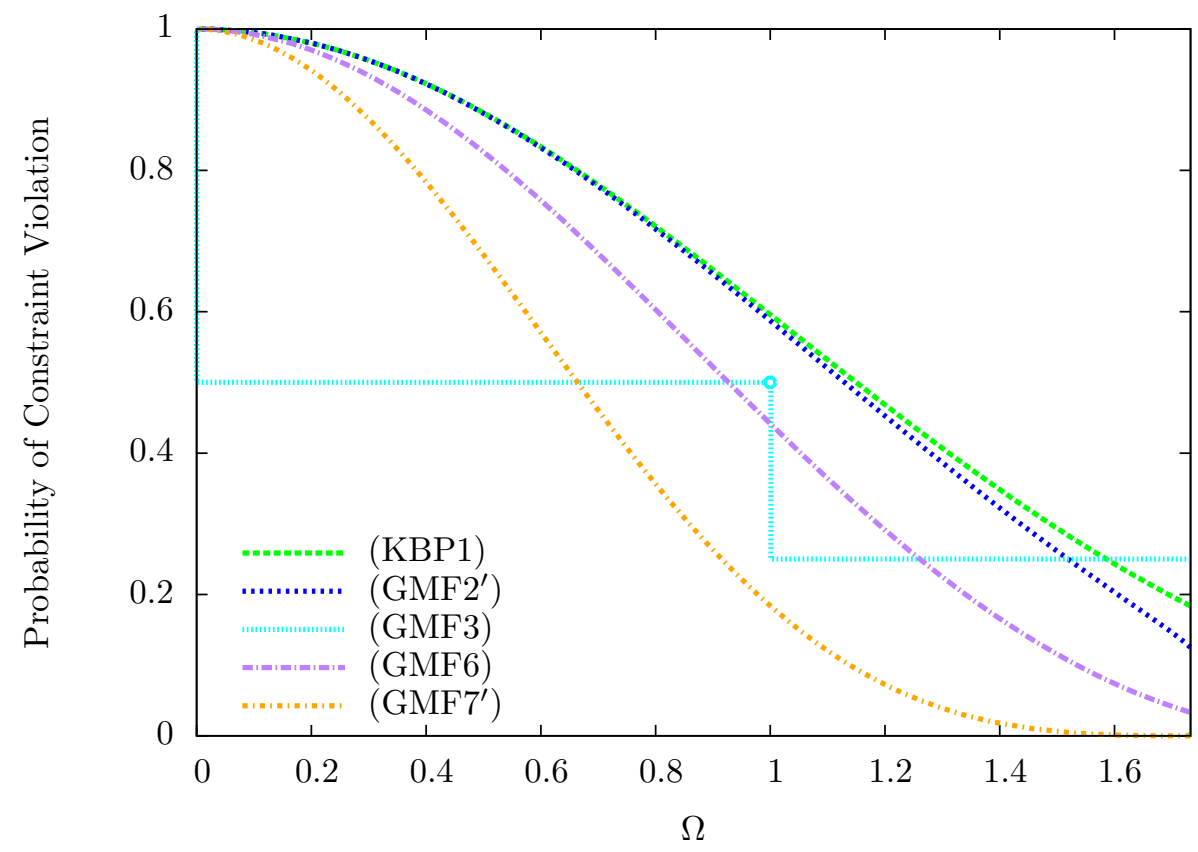

Figure 6: Probability of constraint violation versus $\Omega$ for the interval + ellipsoidal uncertainty set using a priori probabilities of constraint violation for a case with uniform probability distributions, $\left|J_{i}\right|=3$. 


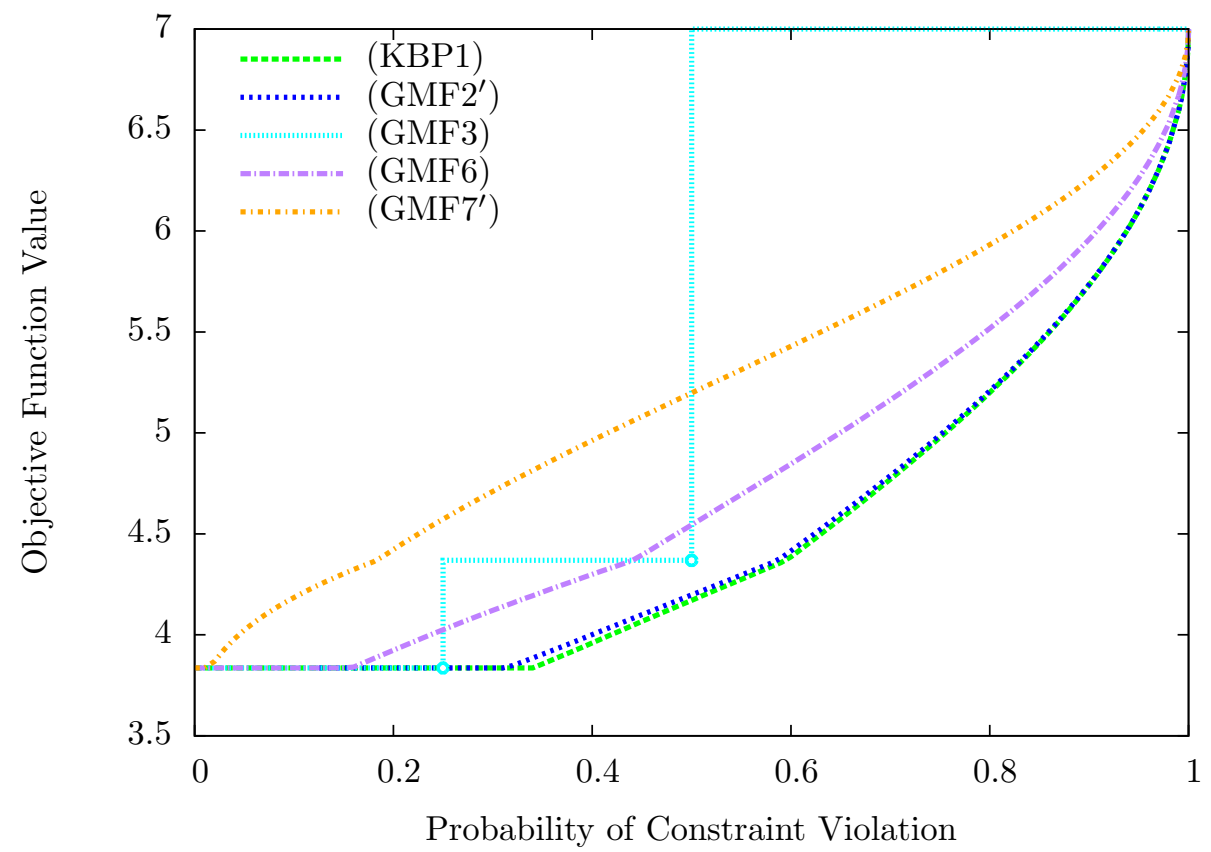

Figure 7: Objective function values from the example MILP at varying probabilities of constraint violation for the interval + ellipsoidal uncertainty set, using a priori bounds for a case with uniform probability distributions for all parameters, $\left|J_{i}\right|=3$. 


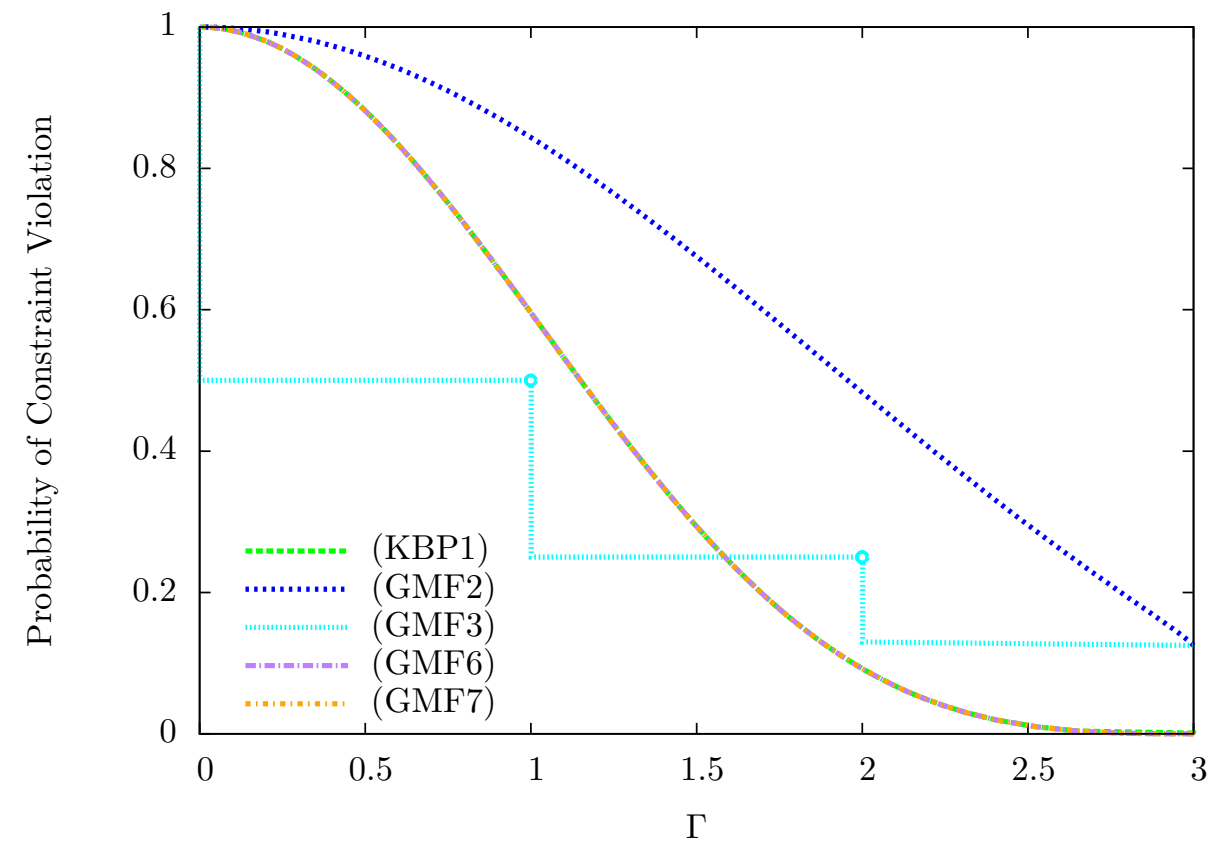

Figure 8: Probability of constraint violation versus $\Gamma$ for the interval + polyhedral uncertainty set using a priori probabilities of constraint violation for a case with uniform probability distributions, $\left|J_{i}\right|=3$. 


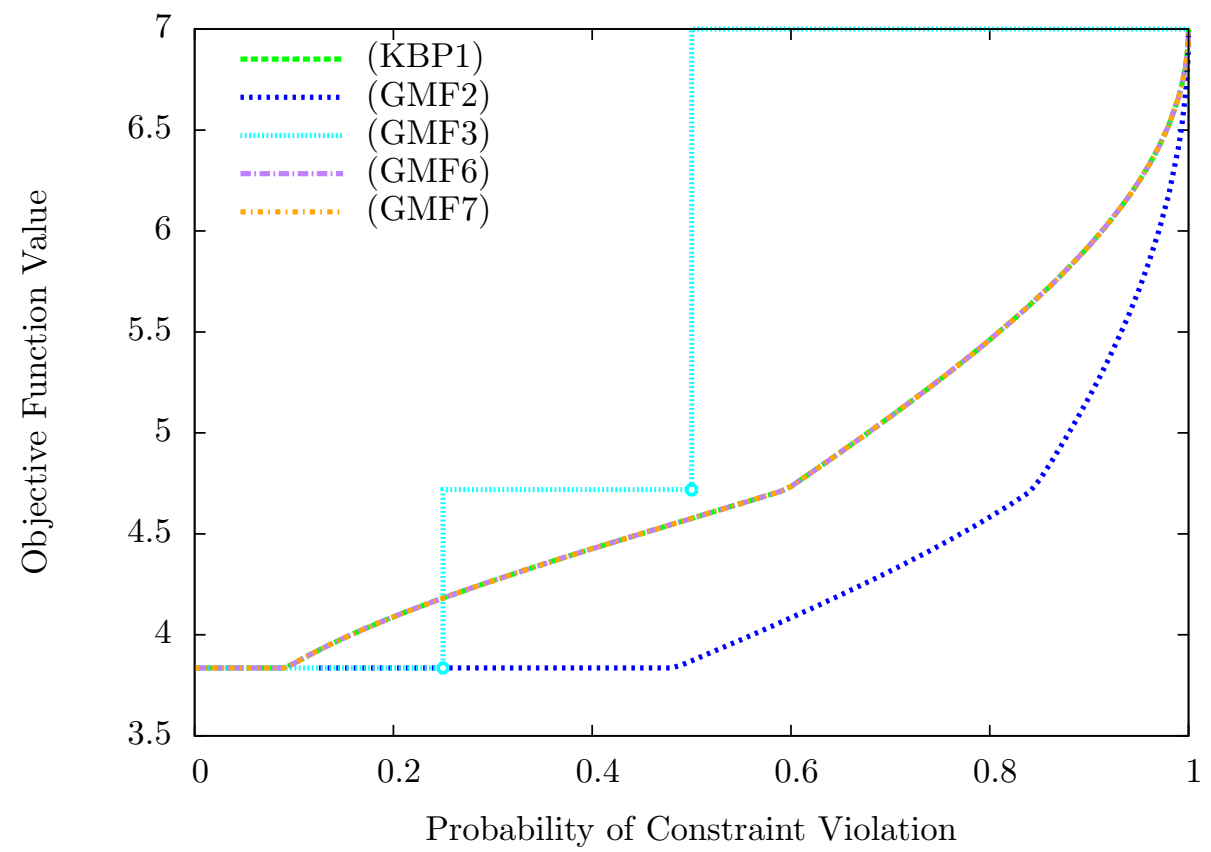

Figure 9: Objective function values from the example MILP at varying probabilities of constraint violation for the interval + polyhedral uncertainty set, using a priori bounds for a case with uniform probability distributions for all parameters, $\left|J_{i}\right|=3$. 
new distribution-dependent bounds. Finally, in the polyhedral uncertainty sets, bounds (KBP1), (GMF6), and (GMF7) behave identically for the case when all distribution functions are identical, as demonstrated in Figures 8 and 9. For these specific problems with $\left|J_{i}\right|=3$, bound (GMF3) is dominant over the distribution-dependent bounds at $\epsilon_{i}^{\text {prio }} \geq 0.25$.

\subsubsection{Case Study 3: Multiple, Unique Symmetric Probability Distributions}

A case in which each uncertain parameter has a unique, symmetric probability distribution highlights the differences in bounds (KBP1), (GMF6), and (GMF7). The objective function and uncertain constraints in the example MILP each have three uncertain parameters, to which we attribute a uniform, triangular, and raised cosine distribution, respectively. With this assumption, the $a$ priori values of $\Omega$ are calculated for the interval + ellipsoidal uncertainty set. The results can be seen in Figures 10 and 11.

For the interval + ellipsoidal uncertainty set, the best performing a priori bound is $\left(\mathrm{GMF}^{\prime}\right)$, except at low $\Omega$ where (GMF3) retains its dominance. Bound $\left(\mathrm{GMF}^{\prime}\right)$ does not outperform (KBP1) as it does in Section 5.1.2. A more distinct difference would be seen when the interval + polyhedral set is used; bound (GMF7) would no longer be equivalent to (KBP1) and (GMF6) as it was in the all-uniform case due to a loss of tightness from using the maximization operator.

\subsubsection{Case Study 4: Asymmetric Probability Distributions}

Finally, it is possible for uncertain parameters to be described by asymmetric probability distributions and included in robust counterpart formulations using the bounds in Section 3.2. The value of these bounds is demonstrated by solving model (49) when $\xi_{i j}, \forall j \in J_{i}$, are described through an asymmetric triangular distribution supported on $[-1,7 / 8]$ with a mode of $1 / 8$ and an expected value of 0 .

The probability of constraint violation and objective function values for the interval + ellipsoidal set using (GMF2), (GMF8), (GMF9), and (GMF10') are shown in Figures 12 and 13. For the interval + ellipsoidal set, each bound provides different performance and levels of conservatism; this would not be seen had an interval + polyhedral set been used, as bounds (GMF8), (GMF9), and (GMF10) would have provided identical $\Gamma$ values since each parameter has the same underlying probability distribution. Each of the distribution-specific bounds improves the tightness relative to (GMF2), which does not utilize any information from the probability distribution but is still applicable with $\mathbf{E}\left[\xi_{i j}\right]=$ 0 . In cases with asymmetry, the tightest bound is unique to the uncertainty set used, as stated in Table 7. For the example MILP, $($ GMF10') is dominant for the interval + ellipsoidal set. To highlight this, the objective function value at a $10 \%$ probability of constraint violation is 4.80 with $\left(\mathrm{GMF} 10^{\prime}\right)$, compared to values of 4.18 with (GMF9) and 3.84 with (GMF2) and (GMF8). 


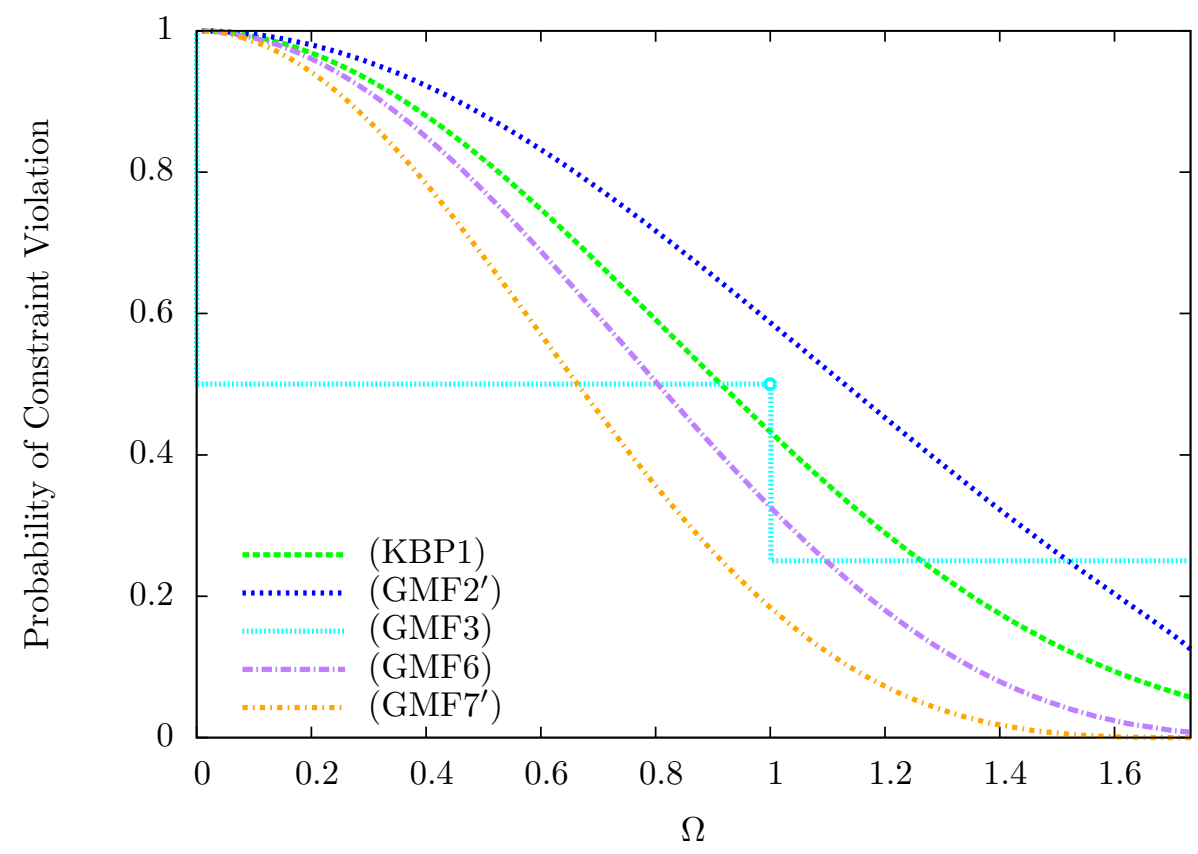

Figure 10: Probability of constraint violation versus $\Omega$ using various a priori bounds for the interval + ellipsoidal uncertainty set with three uncertain parameters described by the uniform, triangular, and raised cosine distributions, respectively. 


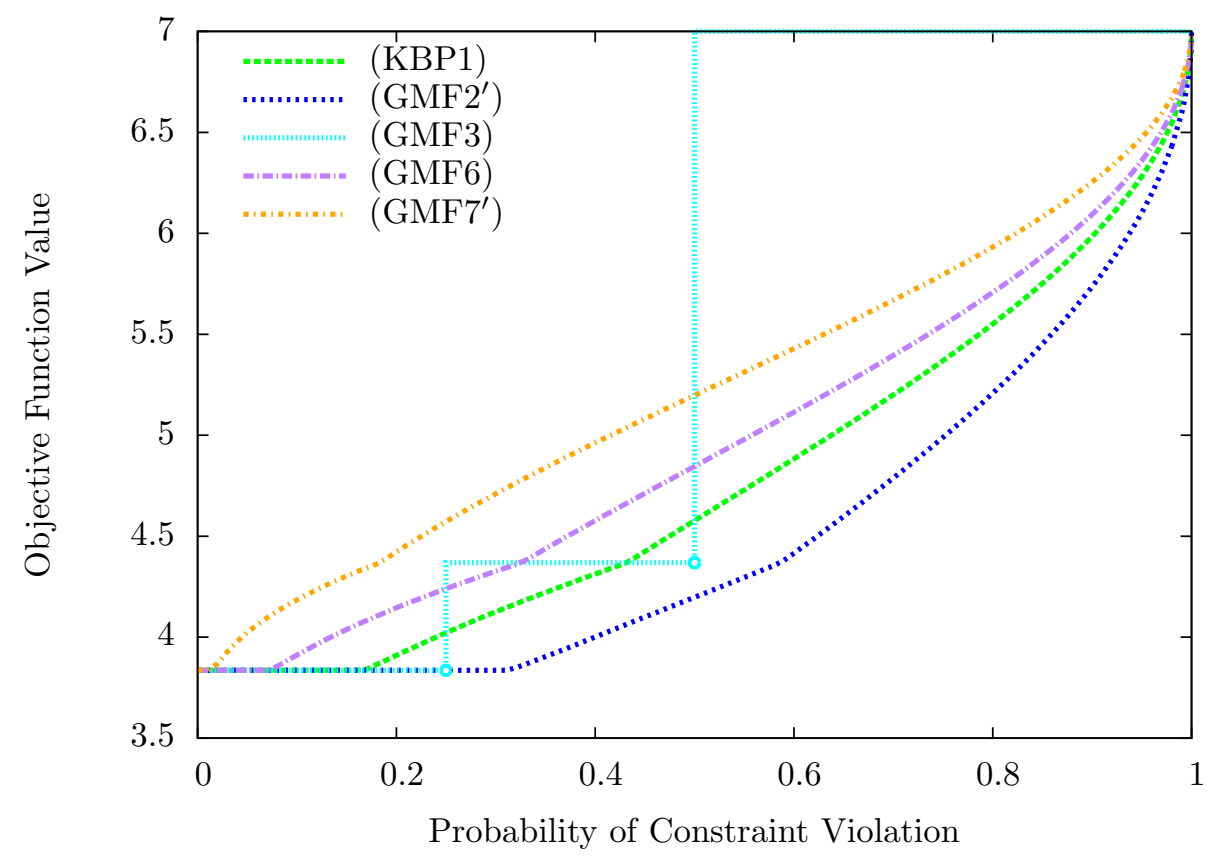

Figure 11: Objective function values from the example MILP at varying probabilities of constraint violation for the interval + ellipsoidal uncertainty set with three uncertain parameters per uncertain constraint and in the uncertain objective function, subject to the uniform, triangular, and raised cosine distributions, respectively. 


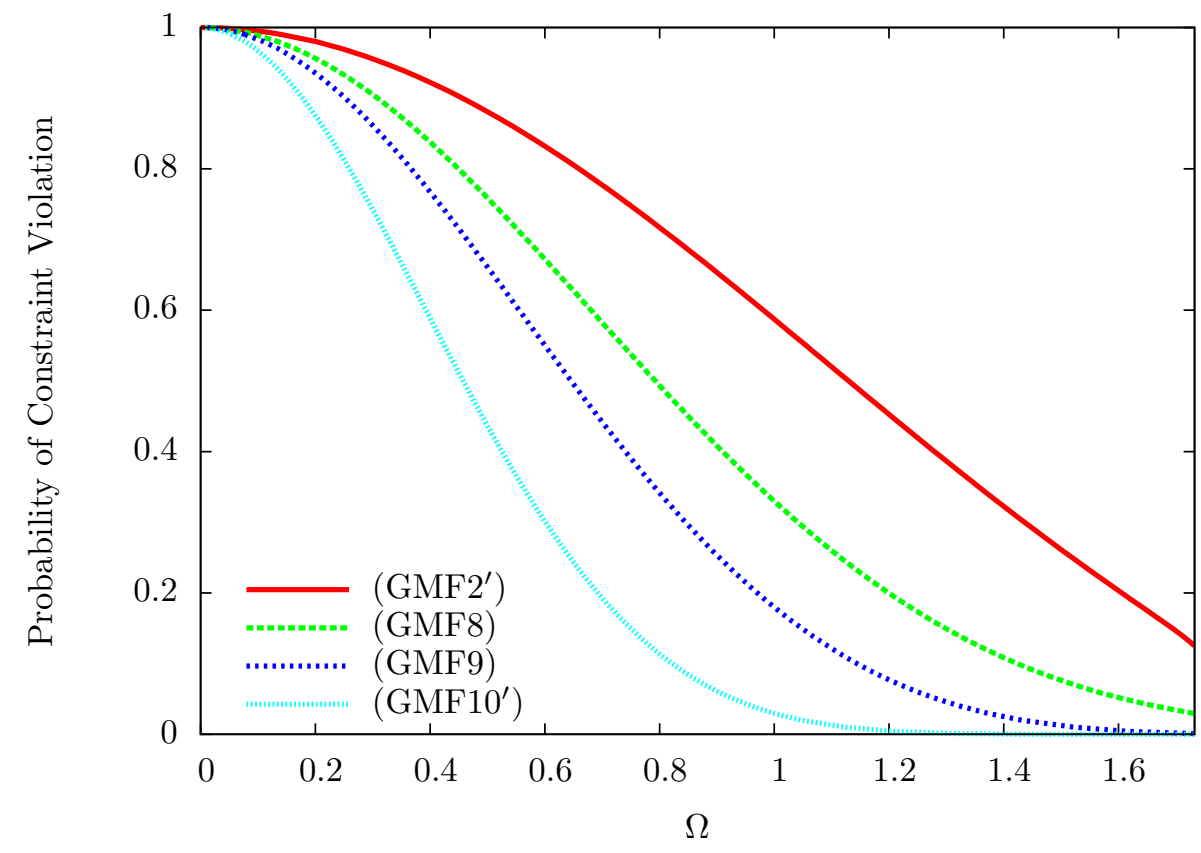

Figure 12: Probability of constraint violations versus $\Omega$ for the interval + ellipsoidal uncertainty set when $\left|J_{i}\right|=3$ and all parameters are described by asymmetric triangular distributions. 


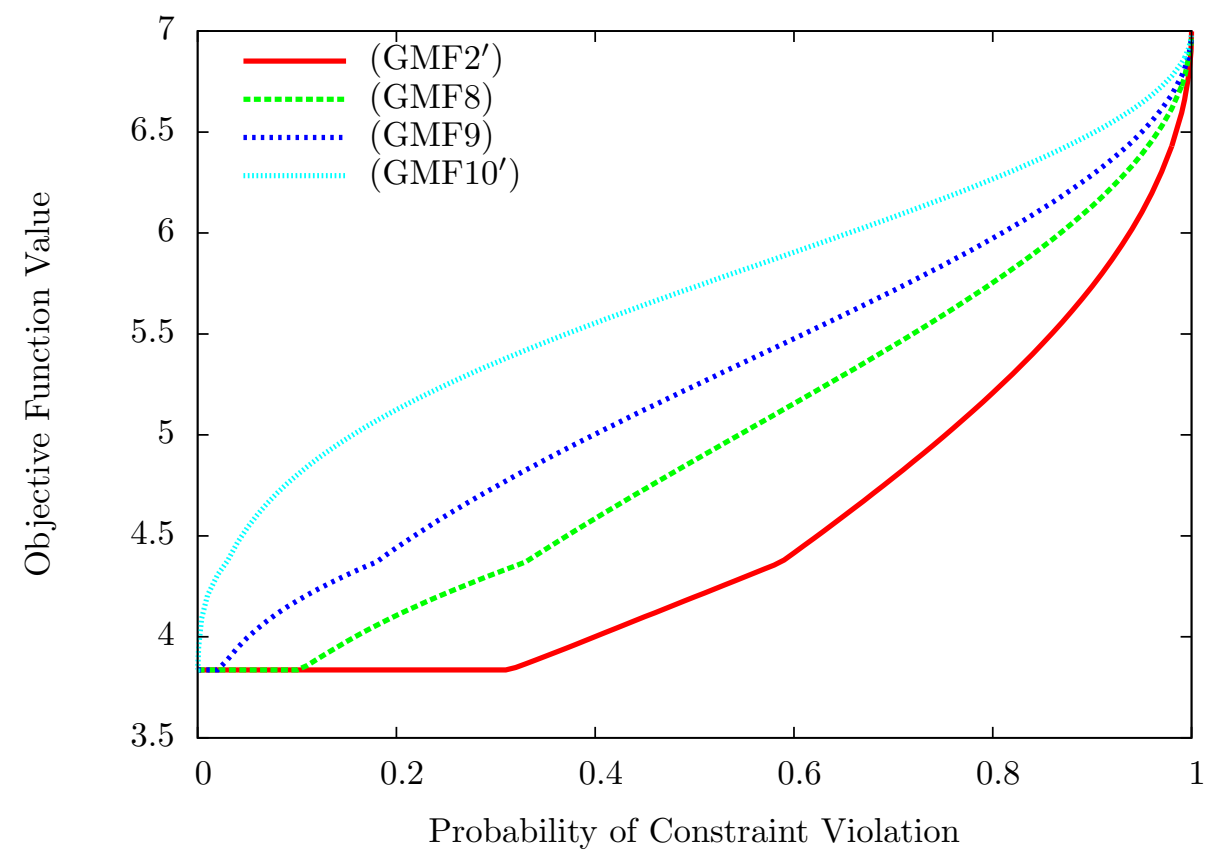

Figure 13: Objective function values as a function of probability of constraint violation for the interval + ellipsoidal uncertainty set with all parameters described by asymmetric triangular distributions, $\left|J_{i}\right|=3$. 


\subsection{Application: Multiperiod Production Planning LP}

Multiperiod production planning is one of many applications of linear programming in which uncertain data can directly impact the optimal solutions. As in Part I (Guzman et al., 2016a), we demonstrate this using an example of planning for a year of production which is expanded from Ravindran (2008) and $\mathrm{Li}$ et al. (2012b). The company wishes to maximize profit, and the year is broken into 12 months with a total budget of $\$ 800,000$ for the year. The model determines the amount of product that should be produced during each month given production costs, selling prices, storage costs, production capacities, and product demands that vary on a monthly basis. It is assumed that the company begins with 500 tons of product in storage, and that the same amount of product will be stored after the 12 months are complete. The company has the ability to sell the product produced in each period immediately, or to store it until it is sold in a future period. An LP formulation of this problem is shown in model (51).

$$
\begin{array}{rlr}
\max _{x, y, z} & \sum_{j}\left(P_{j} z_{j}-C_{j} x_{j}-V_{j} y_{j}\right) & \\
\text { s.t. } & \sum_{j}\left(C_{j} x_{j}+V_{j} y_{j}\right) \leq B & \\
& & \\
& I+x_{1}-\left(y_{1}+z_{1}\right)=0 & \\
& y_{j-1}+x_{j}-\left(y_{j}+z_{j}\right)=0 & \forall j=2, \ldots, 12 \\
& y_{12}=F & \\
& x_{j} \leq U_{j} & \forall j \\
& z_{j} \leq D_{j} & \forall j \\
& x_{j}, y_{j}, z_{j} \geq 0 & \forall j .
\end{array}
$$

In model $(51), x_{j}, y_{j}$, and $z_{j}$ are variables representing the production amount, amount of product in storage, and amount of product sold (tons) in each period, respectively. Parameters $P_{j}, C_{j}$, and $V_{j}$ represent important financial aspects such as the selling price, production cost, and storage cost per ton of product. Each period has a demand $D_{j}$ limiting the amount sold, and a production capacity $U_{j}$ limiting the amount produced. Finally, the total cost of production is limited by the budget $B=\$ 800,000$, while the initial $(I)$ and final $(F)$ amount of product in storage are set such that $I=F=500$ tons. Table 1 shows all parameter values used for each month in (51). 
Table 1: Monthly Planning Data for the LP Production Planning Problem

\begin{tabular}{cccccc}
\hline Period & $\begin{array}{c}\text { Selling Price } \\
(\$ / \text { ton })\end{array}$ & $\begin{array}{c}\text { Production Cost } \\
(\$ / \text { ton })\end{array}$ & $\begin{array}{c}\text { Storage Cost } \\
(\$ / \text { ton })\end{array}$ & $\begin{array}{c}\text { Production Capacity } \\
\text { (tons })\end{array}$ & $\begin{array}{c}\text { Demand } \\
\text { (tons) }\end{array}$ \\
$\mathrm{j}$ & $P_{j}$ & $C_{j}$ & $V_{j}$ & $U_{j}$ & $D_{j}$ \\
\hline 1 & 180 & 20 & 2 & 1500 & 1100 \\
2 & 180 & 25 & 2 & 2000 & 1500 \\
3 & 250 & 30 & 2 & 2200 & 1800 \\
4 & 270 & 40 & 2 & 3000 & 1600 \\
5 & 300 & 50 & 2 & 2700 & 2300 \\
6 & 320 & 60 & 2 & 2500 & 2500 \\
7 & 300 & 80 & 2 & 1900 & 2300 \\
8 & 270 & 55 & 2 & 1500 & 1600 \\
9 & 250 & 45 & 2 & 1300 & 1800 \\
10 & 180 & 30 & 2 & 1100 & 1500 \\
11 & 180 & 30 & 2 & 1200 & 1100 \\
12 & 160 & 25 & 2 & 1400 & 1200 \\
\hline
\end{tabular}

Uncertainty is considered in the objective function alone, specifically regarding the selling price of the product, and thus $\left|J_{i}\right|=12$. The objective function can be transformed such that variable $\zeta$ is maximized with the added constraint

$$
\zeta-\sum_{j} \tilde{P}_{j} z_{j}+\sum_{j}\left(C_{j} x_{j}+V_{j} y_{j}\right) \leq 0 .
$$

Then, a robust counterpart is formulated, where $\tilde{P}_{j}=P_{j}+\xi_{j} \hat{P}_{j}$, with $\tilde{P}_{j}$ representing the actual selling price for each month. It is assumed that the parameters can be described by uniform distributions, with the perturbations $\hat{P}$ limited to $20 \%$ of the nominal values $P_{j}$, at most. The results are shown in Figures 14 through 19.

While the nominal case of the planning problem leads to a profit of $\$ 4,207,706$ the inclusion of uncertainty leads to a worst case value of $\$ 3,206,165$. While the use of traditional robust optimization would cause some to believe that this methodology can only provide a profit of $\$ 3,206,165$, the results for each uncertainty set using a priori bounds certainly prove otherwise. Figure 15 shows the profits for the box uncertainty set; at $10 \%$ probability of constraint violation using bound (GMF6), a profit of $\$ 3,279,854$ occurs. This improvement seems modest in comparison to the interval + ellipsoidal set shown in Figure 17 with $\left(\mathrm{GMF}^{\prime}\right)$, where a $10 \%$ probability of constraint violation yields a profit of $\$ 3,815,628$. In fact, even at a $1 \%$ probability of constraint violation with this bound and uncertainty set, the profit has increased by over $\$ 450,000$ dollars from the worst case. Thus, there is a drastic reduction in conservatism using this a priori bound. The improvements for the interval + polyhedral set are larger than for the box, but are not as drastic as the interval + ellipsoidal set; as Figures 18 and 19 show, (GMF6) and (KBP1) are identical and provide the best values at low $\epsilon_{i}^{\text {prio }}$, with profits of $\$ 3,662,943$ at a $10 \%$ probability of 


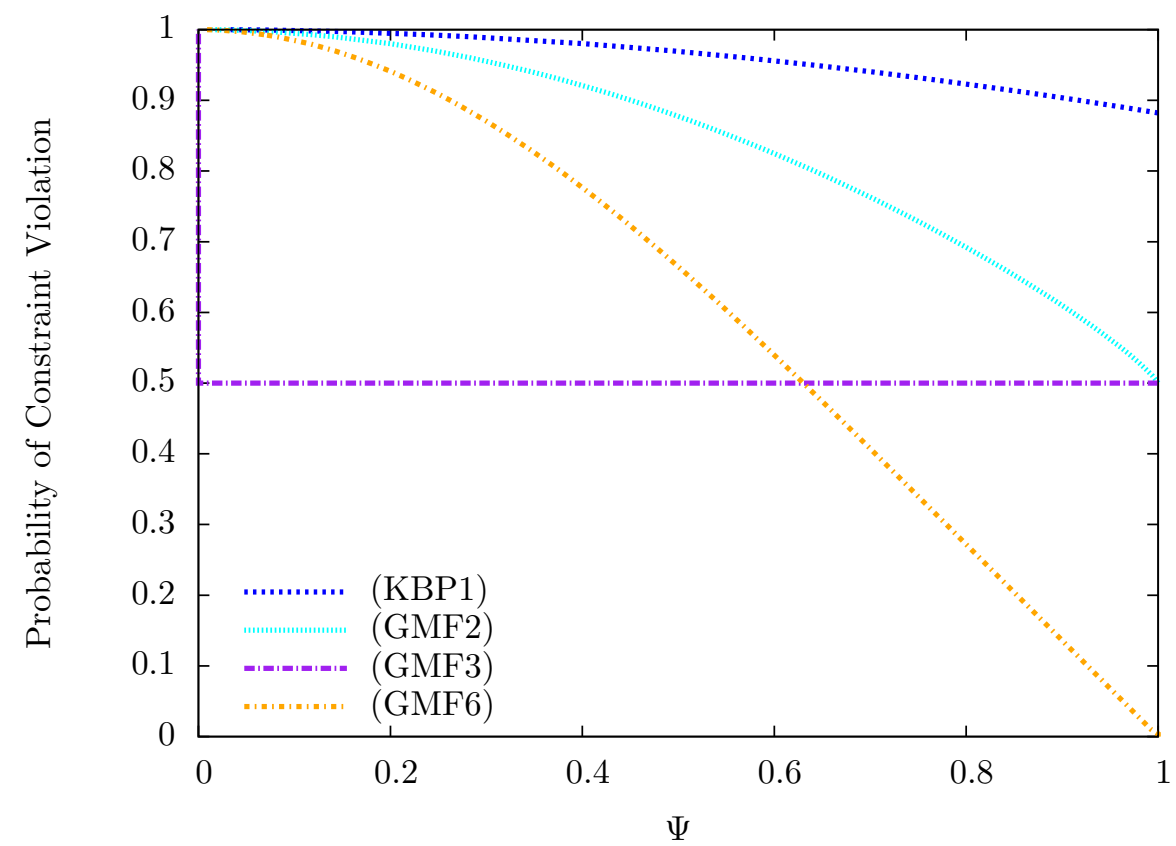

Figure 14: Probability of constraint violation and $\Psi$ values for the box uncertainty set considering uncertainty in the objective function of the multiperiod planning problem, $\left|J_{i}\right|=12$. Uniform distributions are assumed for all uncertain parameters. 


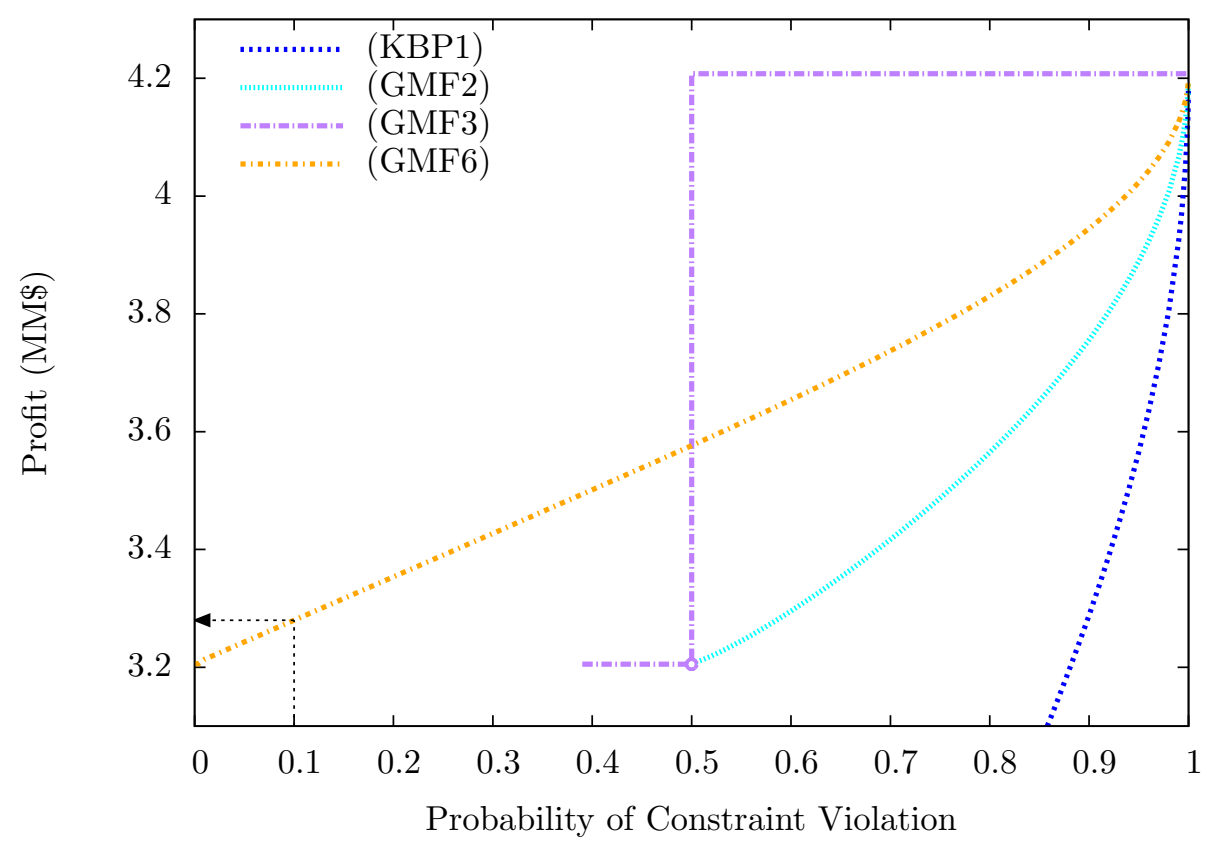

Figure 15: Profit from the multiperiod planning problem as a function of probability of constraint violation for the box uncertainty set with parameters described by uniform distributions, $\left|J_{i}\right|=12$. The profit at a $10 \%$ probability of constraint violation is highlighted with an arrow on the figure. 


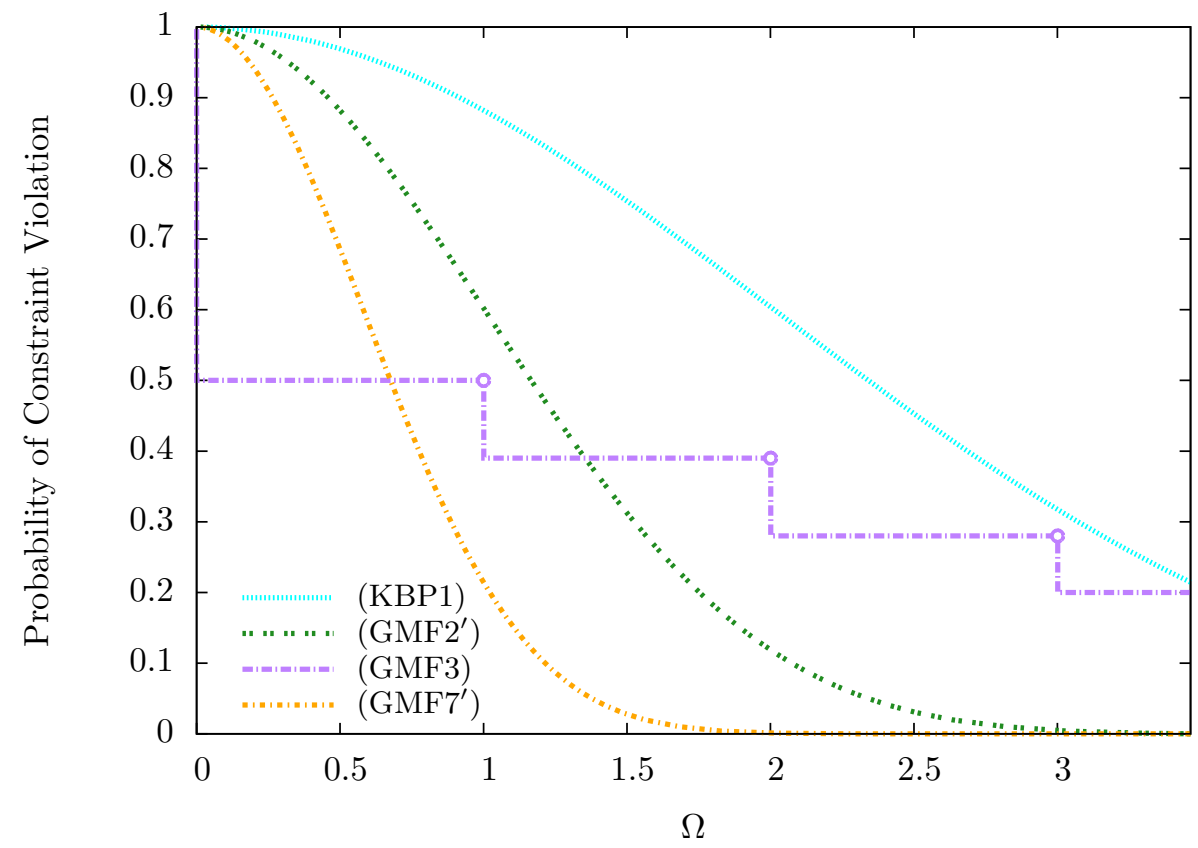

Figure 16: Probability of constraint violation and $\Omega$ values for the interval + ellipsoidal uncertainty set considering uncertainty in the objective function of the multiperiod planning problem, $\left|J_{i}\right|=12$. Uniform distributions are assumed for all uncertain parameters. 


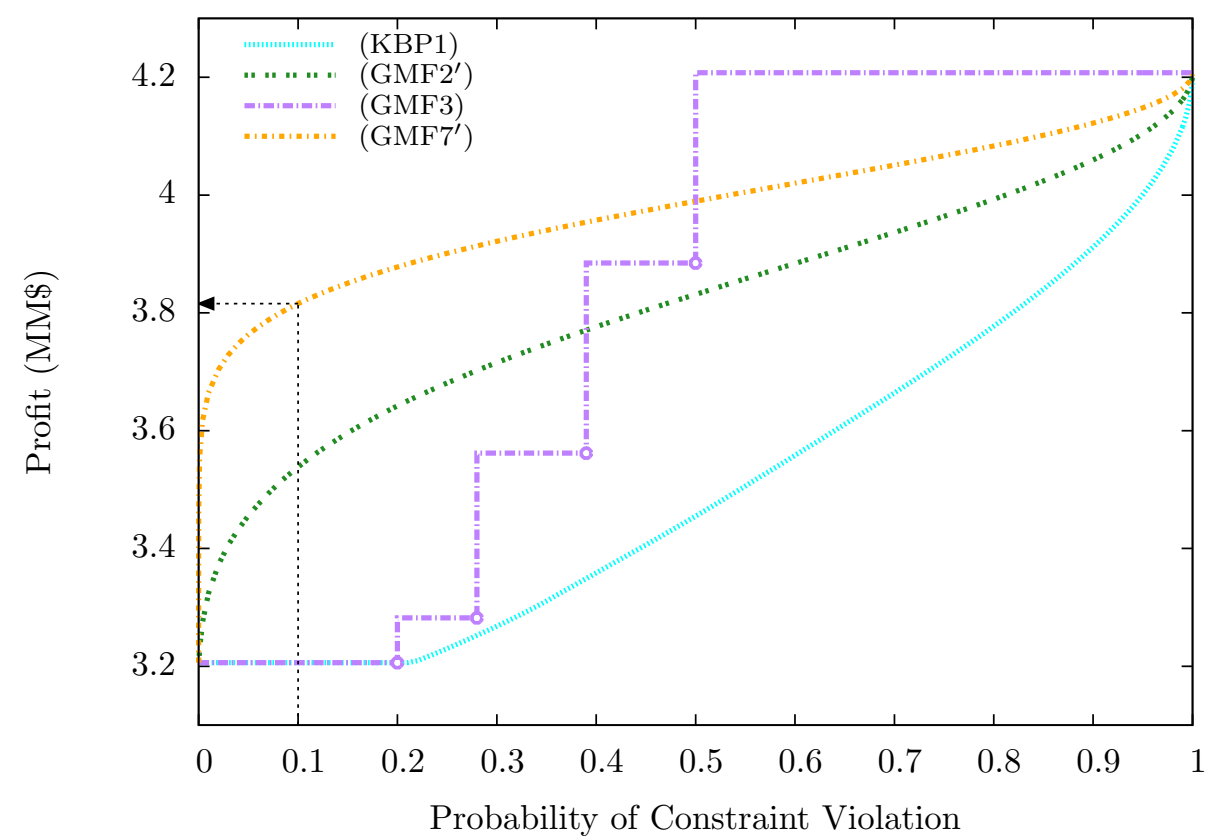

Figure 17: Profit from the multiperiod planning problem as a function of probability of constraint violation for the interval + ellipsoidal uncertainty set with parameters described by uniform distributions, $\left|J_{i}\right|=12$. The profit at a $10 \%$ probability of constraint violation is highlighted with an arrow on the figure. 


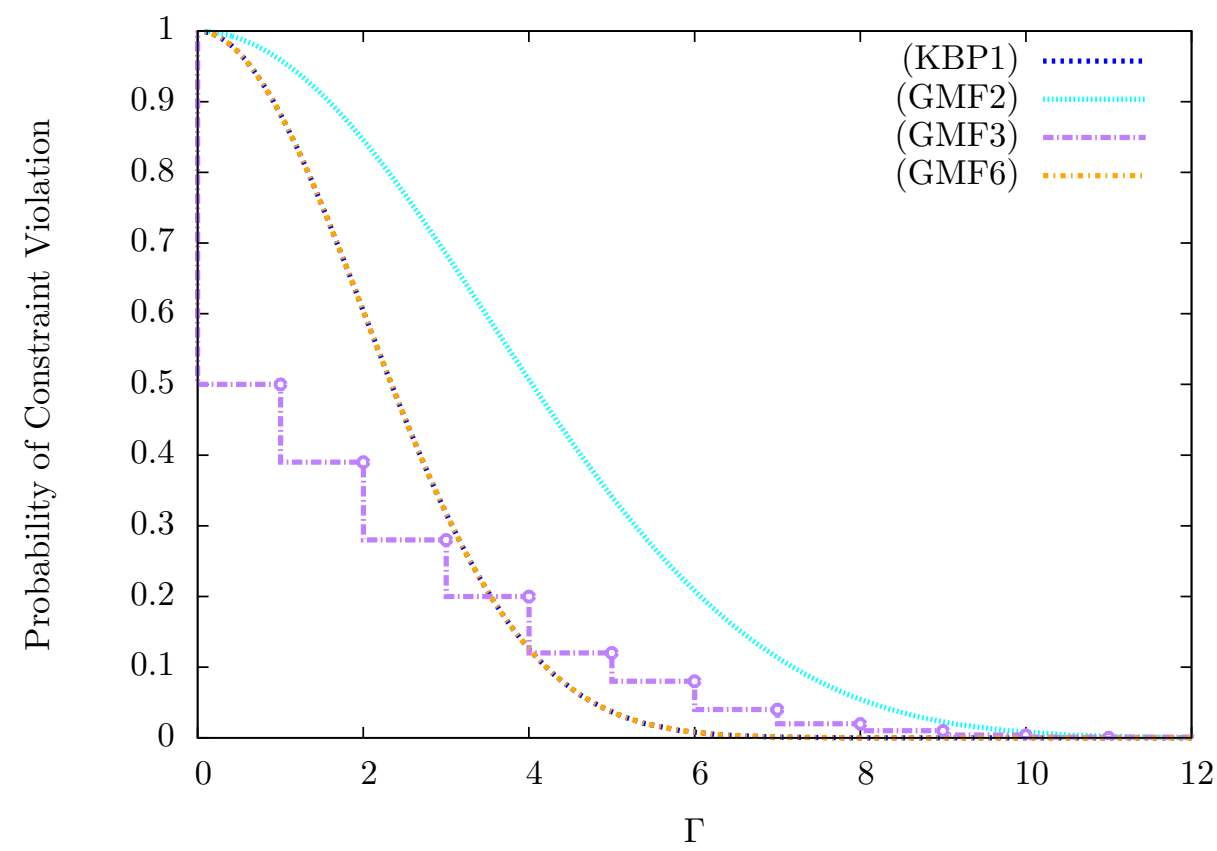

Figure 18: Probability of constraint violation and $\Gamma$ values for the interval + polyhedral uncertainty set considering uncertainty in the objective function of the multiperiod planning problem, $\left|J_{i}\right|=12$. Uniform distributions are assumed for all uncertain parameters. 


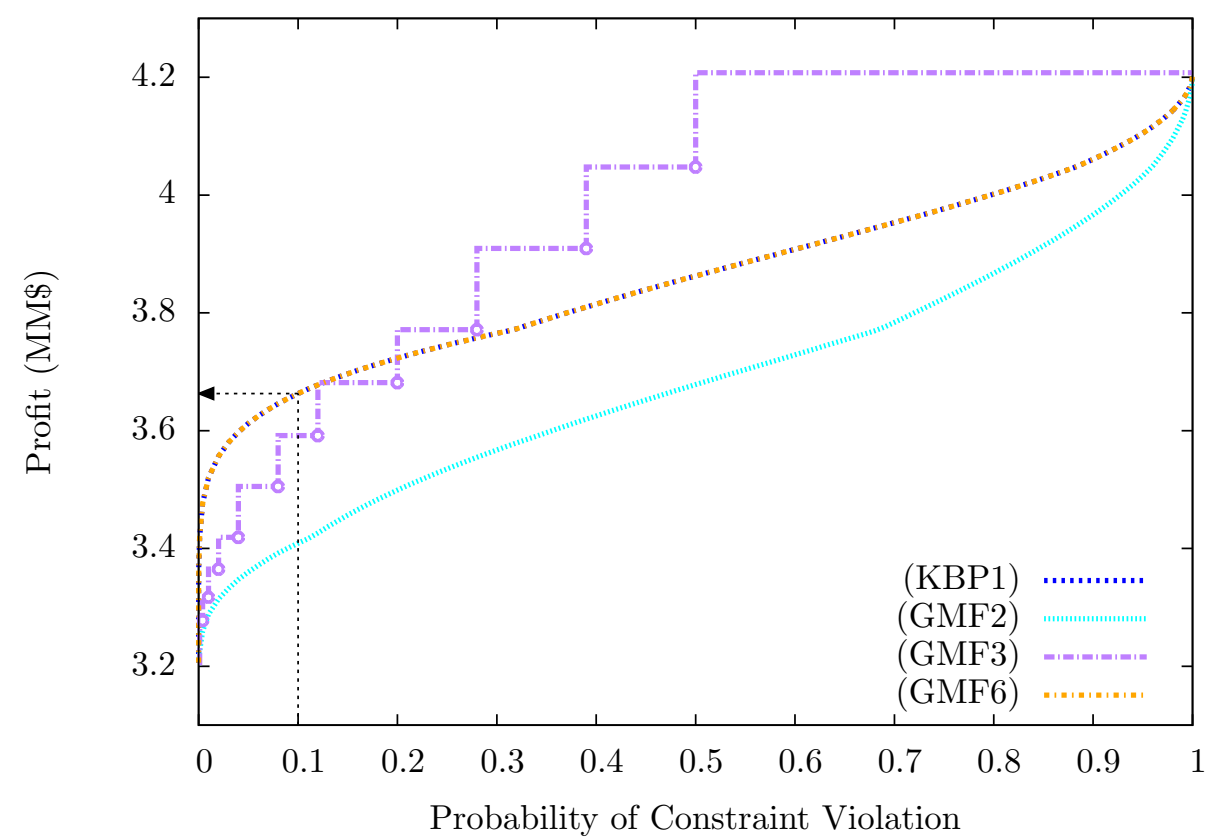

Figure 19: Profit from the multiperiod planning problem as a function of probability of constraint violation for the interval + polyhedral uncertainty set with parameters described by uniform distributions, $\left|J_{i}\right|=12$. The profit at a $10 \%$ probability of constraint violation is highlighted with an arrow on the figure. 
constraint violation. Bound (GMF3) retains its dominance at high probabilities of constraint violation even at a higher $\left|J_{i}\right|$. In Figures 14 and 16, the lowest $\Delta$ values and thus highest profits are found with (GMF3) when $\epsilon_{i}^{\text {prio }} \geq 0.50$ for the box and interval + ellipsoidal sets when $\left|J_{i}\right|=12$. Yet, Figure 18 shows that (GMF3) is dominant when $\epsilon_{i}^{\text {prio }} \geq 0.20$ for the interval + polyhedral sets in the multiperiod planning problem.

As in Part I, the performance of new a priori bounds is shown at six different probabilities of constraint violation ranging from $5 \%$ to $35 \%$ using the price of robustness (PoR) (Bertsimas and Sim, 2004) and normalized price of robustness (NPoR) (Guzman et al., 2016a). The price of robustness (PoR) is calculated as

$$
\operatorname{PoR}=\left|\frac{\zeta_{N}-\zeta_{R}}{\zeta_{N}}\right|,
$$

with $\zeta_{N}$ and $\zeta_{R}$ representing the nominal and robust objective function values. The normalized price of robustness (NPoR) is defined using the gap between the nominal and worst case $\left(\zeta_{W C}\right)$ objective function values in order to reduce the dependence of PoR on the nominal value:

$$
\mathrm{NPoR}=\left|\frac{\zeta_{N}-\zeta_{R}}{\zeta_{N}-\zeta_{W C}}\right| .
$$

The worst case value is simply calculated using a box uncertainty set with $\Psi=1$, and NPoR varies from values of 0 at the nominal case to 1 at the worst case. The value of NPoR can also be referred to as a percentage. The PoR and NPoR are calculated for the interval + ellipsoidal uncertainty set, as seen in Table 2 .

It is clear for the interval + ellipsoidal case that $\left(\mathrm{GMF}^{\prime}\right)$ is the strongest performing bound at values of $\epsilon_{i}^{\text {prio }}$ from $5 \%-35 \%$. At $\epsilon_{i}^{\text {prio }}=0.15$, many of the existing a priori bounds do not even improve upon the worst case (NPoR = $100 \%$ ), while (GMF7') has reduced the NPoR to $35.7 \%$; that is, the solution is much closer to the nominal value than the worst case value. By the time the probability of constraint violation reaches 0.35 , almost $75 \%$ of the profit lost in the worst-case relative to the nominal case has been recovered, and the profit is only $6.36 \%$ lower than the nominal value, as seen by the NPoR and PoR respectively. Interestingly, the two distribution-independent bounds, (GMF2') and (GMF3), are tighter than (KBP1); in fact, $\left(\mathrm{GMF}^{\prime}\right)$ is even tighter than (GMF6) in this range for the planning problem. Thus, there is a benefit to using the simpler, distribution-independent $\left(\mathrm{GMF}^{\prime}\right)$ for the interval + ellipsoidal set over some of the more complicated bounds, although the best performance for this set at low probabilities will involve $\left(\mathrm{GMF}^{\prime}\right)$. 
Table 2: Comparison of existing and new a priori probabilistic bounds for the multiperiod production planning problem using the interval + ellipsoidal uncertainty set.

\begin{tabular}{|c|c|c|c|c|c|c|c|c|c|}
\hline \multirow[b]{2}{*}{$\epsilon_{i}^{\text {prio }}$} & \multicolumn{3}{|c|}{$\left(\mathrm{GMF}^{\prime}\right)$} & \multicolumn{3}{|c|}{$\begin{array}{c}\text { Existing Bounds } \\
\text { (GMF3) }\end{array}$} & \multicolumn{3}{|c|}{ (KBP1) } \\
\hline & $\begin{array}{l}\text { Profit } \\
(\mathrm{MM} \$)\end{array}$ & $\begin{array}{l}\text { PoR } \\
(\%)\end{array}$ & $\begin{array}{l}\text { NPoR } \\
(\%)\end{array}$ & $\begin{array}{l}\text { Profit } \\
(\mathrm{MM} \$)\end{array}$ & $\begin{array}{l}\text { PoR } \\
(\%)\end{array}$ & $\begin{array}{l}\text { NPoR } \\
(\%)\end{array}$ & $\begin{array}{l}\text { Profit } \\
(\mathrm{MM} \$)\end{array}$ & $\begin{array}{l}\text { PoR } \\
(\%)\end{array}$ & $\begin{array}{l}\text { NPoR } \\
(\%)\end{array}$ \\
\hline 0.05 & 3.455 & 17.88 & 75.12 & 3.206 & 23.80 & 100.00 & 3.206 & 23.80 & 100.00 \\
\hline 0.10 & 3.538 & 15.91 & 66.83 & 3.206 & 23.80 & 100.00 & 3.206 & 23.80 & 100.00 \\
\hline 0.15 & 3.596 & 14.53 & 61.05 & 3.206 & 23.80 & 100.00 & 3.206 & 23.80 & 100.00 \\
\hline 0.20 & 3.642 & 13.44 & 56.48 & 3.282 & 22.00 & 92.42 & 3.206 & 23.80 & 100.00 \\
\hline 0.25 & 3.681 & 12.52 & 52.59 & 3.282 & 22.00 & 92.42 & 3.230 & 23.24 & 97.62 \\
\hline 0.30 & 3.715 & 11.70 & 49.15 & 3.562 & 15.35 & 64.48 & 3.268 & 22.33 & 93.82 \\
\hline \multirow[t]{2}{*}{0.35} & 3.747 & 10.95 & 46.00 & 3.562 & 15.35 & 64.48 & 3.311 & 21.32 & 89.55 \\
\hline & \multicolumn{6}{|c|}{$\begin{array}{l}\text { New Bounds } \\
\qquad\left(\text { GMF }^{\prime}\right)\end{array}$} & & & \\
\hline$\epsilon_{i}^{\text {prio }}$ & $\begin{array}{l}\text { Profit } \\
(\mathrm{MM} \$)\end{array}$ & $\begin{array}{l}\text { PoR } \\
(\%)\end{array}$ & $\begin{array}{l}\text { NPoR } \\
(\%)\end{array}$ & $\begin{array}{l}\text { Profit } \\
(\mathrm{MM} \$)\end{array}$ & $\begin{array}{l}\text { PoR } \\
(\%)\end{array}$ & $\begin{array}{l}\text { NPoR } \\
(\%)\end{array}$ & & & \\
\hline 0.05 & 3.381 & 19.65 & 82.57 & 3.763 & 10.56 & 44.38 & & & \\
\hline 0.10 & 3.458 & 17.81 & 74.82 & 3.816 & 9.32 & 39.15 & & & \\
\hline 0.15 & 3.518 & 16.40 & 68.89 & 3.851 & 8.49 & 35.66 & & & \\
\hline 0.20 & 3.567 & 15.23 & 63.99 & 3.878 & 7.84 & 32.93 & & & \\
\hline 0.25 & 3.609 & 14.22 & 59.76 & 3.901 & 7.29 & 30.62 & & & \\
\hline 0.30 & 3.647 & 13.32 & 55.96 & 3.921 & 6.80 & 28.58 & & & \\
\hline 0.35 & 3.682 & 12.49 & 52.47 & 3.940 & 6.36 & 26.72 & & & \\
\hline
\end{tabular}


Table 3: Best performing a priori bounds by objective function value in each computational example considered.

\begin{tabular}{|c|c|c|c|c|c|}
\hline \multirow{2}{*}{ Case Study } & \multirow{2}{*}{$\begin{array}{c}\text { Uncertainty } \\
\text { Set }\end{array}$} & \multicolumn{4}{|c|}{ A Priori Probability of Constraint Violation } \\
\hline & & $5 \%$ & $10 \%$ & $25 \%$ & $50 \%$ \\
\hline \multirow[t]{3}{*}{ Case Study $2^{\mathrm{a}}$} & $\mathrm{B}$ & $($ GMF6 $)=($ GMF7 $)$ & $($ GMF6) $=($ GMF7 $)$ & $($ GMF6) $=($ GMF7 $)$ & (GMF3) \\
\hline & $\mathrm{IE}$ & $\left(\mathrm{GMF}^{\prime}\right)$ & $\left(\right.$ GMF $\left.^{\prime}\right)$ & $\left(\mathrm{GMF}^{\prime}\right)$ & (GMF3) \\
\hline & IP & $\begin{array}{c}(\text { GMF2 })=(\text { GMF3 })=(\text { GMF6 }) \\
=(\text { GMF7 })=(\text { KBP1 })\end{array}$ & $\begin{array}{c}(\mathbf{G M F} \mathbf{6})=(\mathbf{G M F} \mathbf{)}) \\
=(\mathrm{KBP} 1)\end{array}$ & (GMF3) & (GMF3) \\
\hline Case Study $3^{\mathrm{b}}$ & IE & $\left(\mathrm{GMF}^{\prime}\right)$ & $\left(\right.$ GMF7 $\left.^{\prime}\right)$ & $\left(\mathrm{GMF}^{\prime}\right)$ & (GMF3) \\
\hline Case Study $4^{\mathrm{c}}$ & $\mathrm{IE}$ & $\left(\mathrm{GMF}^{\prime} 0^{\prime}\right)$ & $\left(\mathrm{GMF}^{\prime} 0^{\prime}\right)$ & $\left(\mathrm{GMF}^{\prime} 0^{\prime}\right)$ & $\left(\right.$ GMF10 $\left.^{\prime}\right)$ \\
\hline \multirow{3}{*}{$\begin{array}{l}\text { Multiperiod } \\
\text { Planning } \\
\text { Problem }^{\mathrm{d}}\end{array}$} & $\mathrm{B}$ & $($ GMF6) $=($ GMF7 $)$ & $($ GMF6 $)=($ GMF7 $)$ & $($ GMF6) $=($ GMF7 $)$ & (GMF3) \\
\hline & IE & $\left(\mathrm{GMF}^{\prime}\right)$ & $\left(\mathrm{GMF}^{\prime}\right)$ & $\left(\mathrm{GMF}^{\prime}\right)$ & (GMF3) \\
\hline & IP & $(\mathbf{G M F} 6)=(\mathbf{G M F} 7)=(\mathrm{KBP} 1)$ & $\begin{array}{c}(\mathbf{G M F} \mathbf{6})=(\mathbf{G M F} \mathbf{)} \\
=(\mathrm{KBP} 1)\end{array}$ & (GMF3) & (GMF3) \\
\hline
\end{tabular}

${ }^{a}$ Case Study 2 has three uncertain constraints and an uncertain objective function, each with $\left|J_{i}\right|=3$ and uncertain parameters subject to uniform distributions.

${ }^{\mathrm{b}}$ Case Study 3 has three uncertain constraints and an uncertain objective function, each with $\left|J_{i}\right|=3$ and parameters subject to the uniform, triangular, and raised cosine distributions.

${ }^{\mathrm{c}}$ Case Study 4 has three uncertain constraints and an uncertain objective function, each with $\left|J_{i}\right|=3$ and uncertain parameters subject to asymmetric triangular distributions.

d The multiperiod planning problem has an uncertain objective function with $\left|J_{i}\right|=12$ and all parameters subject to uniform distributions. 


\section{Conclusion}

The use of probabilistic bounds in controlling the size of imposed uncertainty sets can significantly enhance the performance of robust counterpart optimization. We derived new a priori bounds on the probability of constraint violation which greatly improve and extend existing methods. The a priori bounds were developed for models with symmetrically or asymmetrically distributed parameters with fully attributed probability distributions. Using symmetric distributions, the new bounds improve the objective function relative to the existing distribution-independent or -dependent bounds at low probabilities of constraint violation for box, ellipsoidal, and interval + ellipsoidal uncertainty sets. The new bounds also enable the usage of fully attributed asymmetric probability distributions for all five uncertainty sets. The conservative approximation of various uncertain parameter distributions with uniform distributions are proposed, with considerable improvement of results relative to unknown probability distributions as demonstrated through a multiperiod planning problem. With the conservatism of traditional robust optimization greatly reduced through the use of these new a priori bounds, the applicability of robust optimization will continue to expand to more areas of optimization involving uncertainty.

\section{Acknowledgments}

The authors gratefully acknowledge financial support from the National Science Foundation (NSF CBET-1158849). This research was conducted with Government support under and awarded by DoD, Air Force Office of Scientific Research, National Defense Science and Engineering Graduate (NDSEG) Fellowship, 32 CFR 168a, which LRM gratefully acknowledges. 
Table 4: Best performing a priori probabilistic bounds on constraint violation: unknown distributions.

\begin{tabular}{|c|c|c|c|c|}
\hline Source & Label & Bound & Applicable $U_{i}$ & Assumptions \\
\hline$\left({ }^{a}\right)$ & $(\mathrm{GMF} 1)^{\mathrm{b}}$ & $\exp \left(\min _{\theta>0}\left\{-\theta \Delta_{i}+\sum_{k \in K_{i}} \ln G_{i k}(\theta)\right\}\right)$ & $\mathrm{B}, \mathrm{E}, \mathrm{IE}, \mathrm{P}, \mathrm{IP}$ & $\xi_{i j} \in[-1,1]$, known bounds on $\mathbf{E}\left[\xi_{i j}\right]$ \\
\hline$(\mathrm{a})$ & $\left(\mathrm{GMF} 1^{\prime}\right)^{\mathrm{c}}$ & $\exp \left(\min _{\theta>0}\left\{-\theta \Delta_{i}+\left|J_{i}\right| \ln \bar{G}_{i}\left(\theta / \sqrt{\left|J_{i}\right|}\right)\right\}\right)$ & $\mathrm{E}, \mathrm{IE}$ & $\xi_{i j} \in[-1,1]$, known bounds on $\mathbf{E}\left[\xi_{i j}\right]$ \\
\hline$(\mathrm{a})$ & $(\mathrm{GMF} 2)^{\mathrm{d}}$ & $\left(\alpha^{\alpha} \beta^{\beta}\right)^{-\delta_{i}^{(1)} / 2}$ & $\mathrm{~B}, \mathrm{E}, \mathrm{IE}, \mathrm{P}, \mathrm{IP}$ & $\xi_{i j} \in[-1,1], \mathbf{E}\left[\xi_{i j}\right]=0$ \\
\hline$(\mathrm{a})$ & $\left(\mathrm{GMF} 2^{\prime}\right)^{\mathrm{d}}$ & $\left(\alpha^{\alpha} \beta^{\beta}\right)^{-\left|J_{i}\right| / 2}$ & $\mathrm{E}, \mathrm{IE}$ & $\xi_{i j} \in[-1,1], \mathbf{E}\left[\xi_{i j}\right]=0$ \\
\hline$(\mathrm{a})$ & $(\text { GMF3) })^{\mathrm{e}}$ & $\frac{1}{2^{\tilde{n}}} \sum_{k=\lceil\tilde{\nu}\rceil}^{\tilde{n}}\left(\begin{array}{l}\tilde{n} \\
k\end{array}\right)$ & $\mathrm{B}, \mathrm{E}, \mathrm{IE}, \mathrm{P}, \mathrm{IP}$ & $\xi_{i j} \in[-1,1], \mathbf{E}\left[\xi_{i j}\right]=0$, symmetric \\
\hline
\end{tabular}

\footnotetext{
${ }^{\mathrm{a}}$ Guzman et al. (2016a)

${ }^{\mathrm{b}} G_{i j}(\theta):=\mu_{i j} \sinh (\theta)+\cosh (\theta)$.

${ }^{\mathrm{c}} \bar{G}_{i}(\theta):=\left(\max _{j \in J_{i}} \mu_{i j}\right) \sinh (\theta)+\cosh (\theta)$.

d $\alpha:=1-\Delta_{i} / \delta_{i}^{(1)}, \beta:=1+\Delta_{i} / \delta_{i}^{(1)}$.

е $\tilde{\nu}:=\left(\Delta_{i}+\tilde{n}\right) / 2, \tilde{n}:=\left|J_{i}\right|+1-\operatorname{sgn} \Delta_{i}$ or $\left|J_{i}\right|-\operatorname{sgn} \Delta_{i}$.
} 
Table 5: Existing and new a priori probabilistic bounds on constraint violation: known, symmetric distributions.

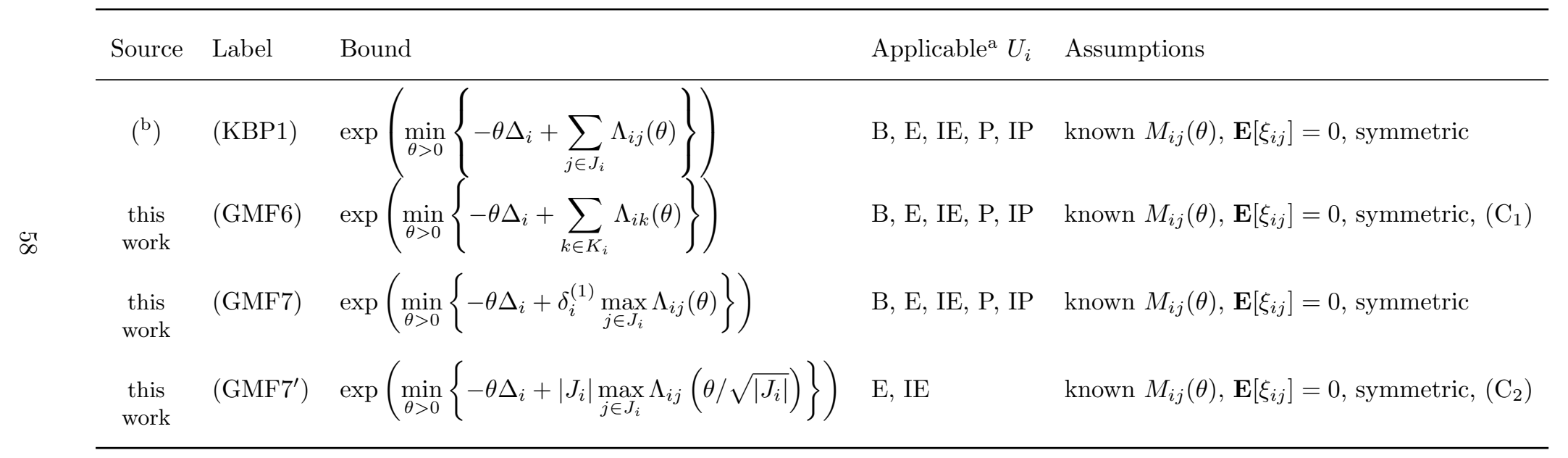

${ }^{\mathrm{a}}$ Bound (KBP1) was formulated for IP uncertainty sets. Its usage was extended to other uncertainty sets in (Li et al., 2012b).

${ }^{\mathrm{b}}$ Kang et al. (2013). 
Table 6: Existing and new a priori probabilistic bounds on constraint violation: known, asymmetric distributions.

\begin{tabular}{|c|c|c|c|c|c|}
\hline Source & Label & Bound $^{\mathrm{a}}$ & & Applicable $U_{i}$ & Assumptions \\
\hline $\begin{array}{l}\text { this } \\
\text { work }\end{array}$ & (GMF8) & $\exp \left(\min _{\theta>0}\right.$ & $\left.\left\{-\theta \Delta_{i}+\sum_{j \in J_{i}} \Lambda_{i j}\left(g_{i j} \theta\right)\right\}\right)$ & $\mathrm{B}, \mathrm{E}, \mathrm{IE}, \mathrm{P}, \mathrm{IP}$ & known $M_{i j}(\theta), \mathbf{E}\left[\xi_{i j}\right]=0, g_{i j}$ exists \\
\hline $\begin{array}{l}\text { this } \\
\text { work }\end{array}$ & (GMF9) & $\exp \left(\min _{\theta>0}\right.$ & $\left.\left\{-\theta \Delta_{i}+\sum_{k \in K_{i}} \Lambda_{i k}\left(g_{i k} \theta\right)\right\}\right)$ & $\mathrm{B}, \mathrm{E}, \mathrm{IE}, \mathrm{P}, \mathrm{IP}$ & known $M_{i j}(\theta), \mathbf{E}\left[\xi_{i j}\right]=0, g_{i j}$ exists, $\left(\mathrm{C}_{1}\right)$ \\
\hline $\begin{array}{l}\text { this } \\
\text { work }\end{array}$ & (GMF10) & $\exp \left(\min _{\theta>0}\right.$ & $\left.\left\{-\theta \Delta_{i}+\delta_{i}^{(1)} \max _{j \in J_{i}} \Lambda_{i j}\left(g_{i j} \theta\right)\right\}\right)$ & $\mathrm{B}, \mathrm{E}, \mathrm{IE}, \mathrm{P}, \mathrm{IP}$ & known $M_{i j}(\theta), \mathbf{E}\left[\xi_{i j}\right]=0, g_{i j}$ exists \\
\hline $\begin{array}{l}\text { this } \\
\text { work }\end{array}$ & $\left(\mathrm{GMF} 10^{\prime}\right)$ & $\exp \left(\min _{\theta>0}\right.$ & $\left.\left\{-\theta \Delta_{i}+\left|J_{i}\right| \max _{j \in J_{i}} \Lambda_{i j}\left(g_{i j} \theta / \sqrt{\left|J_{i}\right|}\right)\right\}\right)$ & $\mathrm{E}, \mathrm{IE}$ & known $M_{i j}(\theta), \mathbf{E}\left[\xi_{i j}\right]=0, g_{i j}$ exists, $\left(\mathrm{C}_{2}\right)$ \\
\hline
\end{tabular}

${ }^{\mathrm{a}} g_{i j}= \pm 1$, see $(22)$. 
Table 7: Tightest a priori bounds by case.

\begin{tabular}{|c|c|c|c|}
\hline \multirow{2}{*}{ Available Information } & \multicolumn{3}{|c|}{ Uncertainty Set } \\
\hline & $\mathrm{B}$ & $\mathrm{E}, \mathrm{IE}$ & $\mathrm{P}, \mathrm{IP}$ \\
\hline $\begin{array}{l}\xi_{i j} \text { bounded, known bounds } \\
\text { on } \mathbf{E}\left[\xi_{i j}\right]\end{array}$ & (GMF1) & $(\mathrm{GMF1})$ or $\left(\mathrm{GMF1}^{\prime}\right)$ & (GMF1) \\
\hline$\xi_{i j}$ bounded, $\mathbf{E}\left[\xi_{i j}\right]$ known & (GMF2) & $\left(\mathrm{GMF}^{\prime}\right)$ & (GMF2) \\
\hline$\xi_{i j}$ bounded and symmetric & (GMF3) & $\left(\right.$ GMF2 $\left.^{\prime}\right)$ or (GMF3) & (GMF3) \\
\hline $\begin{array}{l}\text { bell-shaped bounded distri- } \\
\text { butions (conservatively ap- } \\
\text { plying uniform distributions) }\end{array}$ & $\begin{array}{c}(\text { GMF3) or } \\
(\text { GMF6) }=(\text { GMF7) }\end{array}$ & (GMF3) or $\left(\mathrm{GMF}^{\prime}\right)$ & $\begin{array}{c}(\text { GMF3 }) \text { or } \\
(\text { GMF6 })=(\text { GMF7 })=(\text { KBP1 })\end{array}$ \\
\hline $\begin{array}{l}\xi_{i j} \text { distribution known, sym- } \\
\text { metric }\end{array}$ & $\left(\right.$ GMF3) or $(\text { GMF6 })^{\mathrm{a}}$ & $\begin{array}{l}\left(\text { GMF3) or }(\text { GMF6 })^{\mathrm{a}}\right. \\
\text { or }\left(\mathbf{G M F}^{\prime}\right)^{\mathrm{a}}\end{array}$ & $\begin{array}{c}\text { (GMF3) or } \\
(\text { GMF6) })^{\mathrm{a}}=(\mathrm{KBP} 1)\end{array}$ \\
\hline $\begin{array}{l}\xi_{i j} \text { distribution known, } \\
\text { asymmetric }\end{array}$ & $(\text { GMF9) })^{\mathrm{b}}$ & $(\text { GMF9) })^{\mathrm{b}}$ or $\left(\mathrm{GMF}^{\prime} 0^{\prime}\right)^{\mathrm{b}}$ & $\left(\right.$ GMF8) $=(\text { GMF9 })^{\mathrm{b}}$ \\
\hline
\end{tabular}


Table A.1: Definition of uncertainty sets.

\begin{tabular}{cclc}
\hline$U^{i}$ & Parameter $^{\mathrm{a}}$ & Definition & $\max \Delta_{i}^{\mathrm{b}}$ \\
\hline Box & $\Psi_{i}$ & $U_{i}^{\infty}=\left\{\xi_{i}:\left\|\xi_{i}\right\|_{\infty} \leq \Psi_{i}\right\}$ & 1 \\
Ellipsoidal & $\Omega_{i}$ & $U_{i}^{2}=\left\{\xi_{i}:\left\|\xi_{i}\right\|_{2} \leq \Omega_{i}\right\}$ & $\sqrt{\left|J_{i}\right|}$ \\
Interval + Ellipsoidal & $\Omega_{i}$ & $U_{i}^{2 \cap \infty}=\left\{\xi_{i}:\left\|\xi_{i}\right\|_{2} \leq \Omega_{i},\left\|\xi_{i}\right\|_{\infty} \leq 1\right\}$ & $\sqrt{\left|J_{i}\right|}$ \\
Polyhedral & $\Gamma_{i}$ & $U_{i}^{1}=\left\{\xi_{i}:\left\|\xi_{i}\right\|_{1} \leq \Gamma_{i}\right\}$ & $\left|J_{i}\right|$ \\
Interval + Polyhedral & $\Gamma_{i}$ & $U_{i}^{1 \cap \infty}=\left\{\xi_{i}:\left\|\xi_{i}\right\|_{1} \leq \Gamma_{i},\left\|\xi_{i}\right\|_{\infty} \leq 1\right\}$ & $\left|J_{i}\right|$ \\
\hline
\end{tabular}

${ }^{\text {a }}$ Original notation of parameter used here; $\Delta_{i}$ used for the unspecified case.

b Applicable when $\xi_{i j} \in[-1,1], \forall j \in J_{i}$. The set $J_{i}$ contains the indices of uncertain parameters in constraint $i$.

\section{Appendix A. Reference Tables}

Table A.1 summarizes the various uncertainty sets with respect to $\xi_{i}$. Table A. 2 presents the robust counterparts of uncertain constraints. Table A.3 summarizes characteristics of $\delta_{i j}$ and defines parameters $\delta_{i}^{(1)}$ and $\delta_{i}^{(2)}$, the former of which is referenced in some of the probabilistic bounds. Table A.4 displays information on common probability distributions, including their moment generating functions, derivatives of their cumulant generating functions, and their relationship with qualifying conditions $\left(\mathrm{C}_{1}\right)$ and $\left(\mathrm{C}_{2}\right)$. 
Table A.2: Robust counterparts of constraint $i$ by uncertainty set type.

\begin{tabular}{ll}
$U_{i} \quad$ & $\sum_{j} a_{i j} x_{j}+\max _{\xi_{i} \in U_{i}}\left\{\sum_{j \in J_{i}} \xi_{i j} \hat{a}_{i j} x_{j}\right\} \leq b_{i}$ \\
\hline $\mathrm{B}$ & $\sum_{j} a_{i j} x_{j}+\Psi_{i} \sum_{j \in J_{i}} \hat{a}_{i j}\left|x_{j}\right| \leq b_{i}$ \\
$\mathrm{E}$ & $\sum_{j} a_{i j} x_{j}+\Omega_{i} \sqrt{\sum_{j \in J_{i}} \hat{a}_{i j}^{2} x_{j}^{2}} \leq b_{i}$ \\
$\mathrm{IE} \quad \sum_{j} a_{i j} x_{j}+\sum_{j \in J_{i}} \hat{a}_{i j}\left|x_{j}-z_{i j}\right|+\Omega_{i} \sqrt{\sum_{j \in J_{i}} \hat{a}_{i j}^{2} z_{i j}^{2}} \leq b_{i}$ \\
$\mathrm{P} \quad(1) \sum_{j} a_{i j} x_{j}+\Gamma_{i} z_{i} \leq b_{i} \quad(2) z_{i} \geq \hat{a}_{i j}\left|x_{j}\right|, \forall j \in J_{i}$ \\
$\mathrm{IP} \quad(1) \sum_{j} a_{i j} x_{j}+\sum_{j \in J_{i}} p_{i j}+\Gamma_{i} z_{i} \leq b_{i} \quad(2) z_{i} \geq 0$ \\
& $(3) z_{i}+p_{i j} \geq \hat{a}_{i j}\left|x_{j}\right|, \forall j \in J_{i} \quad(4) p_{i j} \geq 0, \forall j \in J_{i}$
\end{tabular}

Table A.3: Associated parameters of uncertainty sets. ${ }^{\text {a }}$

\begin{tabular}{|c|c|c|}
\hline$U_{i}$ & Definition of $\delta_{i}$ & $\left\|\delta_{i}\right\|_{1} \leq \delta_{i}^{(1)}$ \\
\hline B & $\delta_{i j}:=\frac{\hat{a}_{i j} x_{j}}{\sum_{j} \hat{a}_{i j}\left|x_{j}\right|}$ & $\sum_{j}\left|\delta_{i j}\right| \leq 1$ \\
\hline $\mathrm{E}$ & $\delta_{i j}:=\frac{\hat{a}_{i j} x_{j}}{\sqrt{\sum_{j} \hat{a}_{i j}^{2} x_{j}^{2}}}$ & $\sum_{j}\left|\delta_{i j}\right| \leq \sqrt{\left|J_{i}\right|^{\mathrm{c}}} \quad \sqrt{\sum_{j} \delta_{i j}^{2}}=1$ \\
\hline $\mathrm{IE}^{\mathrm{b}}$ & $\delta_{i j}:=\frac{\hat{a}_{i j} z_{i j}}{\sqrt{\sum_{j} \hat{a}_{i j}^{2} z_{i j}^{2}}}$ & $\sum_{j}\left|\delta_{i j}\right| \leq \sqrt{\left|J_{i}\right|^{c}} \quad \sqrt{\sum_{j} \delta_{i j}^{2}}=1$ \\
\hline $\mathrm{P}$ & $\delta_{i j}:=\frac{\hat{a}_{i j} x_{j}}{\max _{j} \hat{a}_{i j}\left|x_{j}\right|}$ & $\sqrt{\sum_{j} \delta_{i j}^{2}} \leq \sqrt{\left|J_{i}\right|}$ \\
\hline$I P^{b, d}$ & $\delta_{i j}:=\min \left\{1, \frac{\hat{a}_{i j} \mid}{\hat{a}_{i r} \mid}\right.$ & $\sqrt{\sum_{j} \delta_{i j}^{2}} \leq \sqrt{\left|J_{i}\right|}$ \\
\hline
\end{tabular}

${ }^{\text {a }}$ Note that all $j \in J_{i}$, and that for all uncertainty sets, $\delta_{i j} \in[-1,1]$. Notation uses vector $\delta_{i}$ with elements $\delta_{i j}$.

b Assumes that probability distributions are bounded.

c Follows from the Cauchy-Schwarz inequality.

${ }^{\mathrm{d}}$ Index $r$ is the index of the $\left(\max \left\{1,\left\lceil\Gamma_{i}\right\rceil\right\}\right)$ th largest $\hat{a}_{i j}\left|x_{j}\right|$. 
Table A.4: Relevant information on common probability distributions.

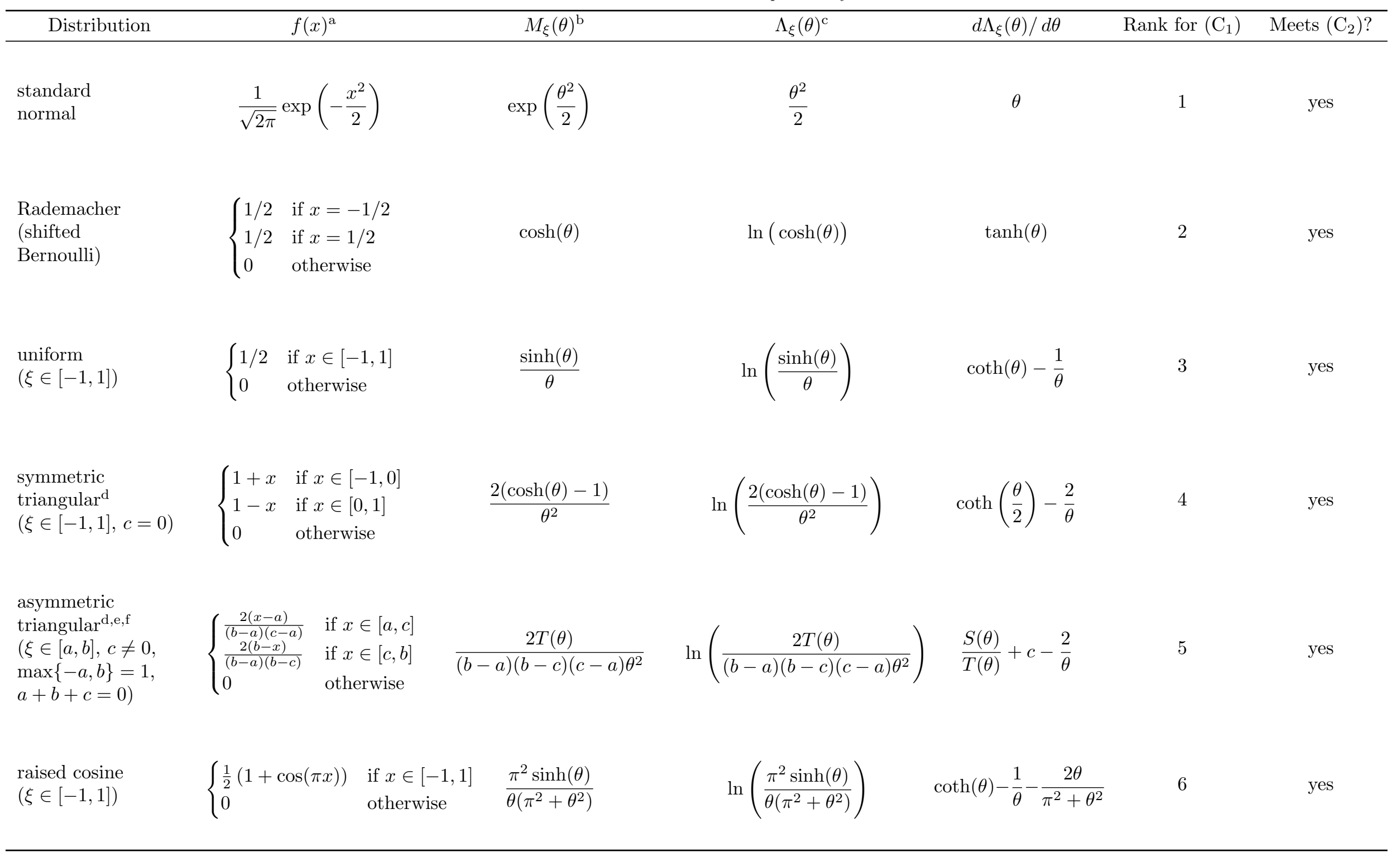

${ }^{\text {a }} f(x)$ is the probability density function or probability mass function of $\xi$.

b $M_{\xi}(\theta)=\mathbf{E}\left[e^{\theta \xi}\right]$ is the moment generating function of $\xi$.

${ }^{\mathrm{c}} \Lambda_{\xi}(\theta)=\ln \mathbf{E}\left[e^{\theta \xi}\right]$ is the cumulant generating function of $\xi$.

${ }^{\mathrm{d}}$ Parameter $c$ is the mode of a triangular distribution.

e Rank for $\left(\mathrm{C}_{1}\right)$ and satisfaction of $\left(\mathrm{C}_{2}\right)$ hold in the asymmetric case for $\Lambda_{i j}^{*}(\theta)=\Lambda_{i j}(g \theta)$ given that $g=-\operatorname{sgn} c$ by $(22)$.

${ }^{\mathrm{f}} T(\theta):=(c-a) \exp (b \theta)-(b-a) \exp (c \theta)+(b-c) \exp (a \theta) . S(\theta):=(b-c)(c-a)(\exp (b \theta)-\exp (a \theta))$. 


\section{Appendix B. Lemmas for theorems in Section 3}

In deriving the probabilistic bounds of Section 3, three lemmas were required. Item (i) of Lemma 1 was noted by Hoeffding (1963) as a tighter preliminary to the well-known Hoeffding's lemma, while item (ii) is relevant to discussion in Section 3.

Lemma 1. Given the random variable $\xi$ is subject to a probability distribution that is supported on the bounded interval $[a, b]$, and given that its moment generating function $M_{\xi}(\theta)=\mathbf{E}\left[e^{\theta \xi}\right]$ exists, then for any real number $\theta$,

(i) $\mathbf{E}\left[e^{\theta \xi}\right] \leq \frac{b-\mathbf{E}[\xi]}{b-a} e^{\theta a}+\frac{\mathbf{E}[\xi]-a}{b-a} e^{\theta b}$

(ii) If $\xi$ is subject to a symmetric distribution, $a=-1$, and $b=1$, then

$$
\frac{d^{n}}{d \theta^{n}} \mathbf{E}\left[e^{\theta \xi}\right] \leq \begin{cases}\cosh (\theta) & \text { if } n \text { is even } \\ \sinh (|\theta|) & \text { if } n \text { is odd. }\end{cases}
$$

Proof. For (i), see Lemma 1, Guzman et al. (2016a).

For (ii),

$$
\begin{aligned}
& \frac{d^{n}}{d \theta^{n}} \mathbf{E}\left[e^{\theta \xi}\right]=\frac{d^{n}}{d \theta^{n}} \mathbf{E}\left[\sum_{k=0}^{\infty} \frac{\theta^{k} \xi^{k}}{k !}\right] \\
& =\frac{d^{n}}{d \theta^{n}} \sum_{k=0}^{\infty} \frac{\theta^{k} \mathbf{E}\left[\xi^{k}\right]}{k !} \\
& =\sum_{k=n}^{\infty} \frac{\theta^{k-n} \mathbf{E}\left[\xi^{k}\right]}{(k-n) !} \\
& =\sum_{k=0}^{\infty} \frac{\theta^{k} \mathbf{E}\left[\xi^{k+n}\right]}{k !} \\
& \stackrel{1}{=} \begin{cases}\sum_{k=0}^{\infty} \frac{\theta^{2 k} \mathrm{E}\left[\xi^{2 k+n}\right]}{(2 k) !} & \text { if } n \text { is even } \\
\sum_{k=0}^{\infty} \frac{\theta^{2 k+1} \mathbf{E}\left[\xi^{2 k+1+n}\right]}{(2 k+1) !} & \text { if } n \text { is odd }\end{cases} \\
& \stackrel{2}{\leq} \begin{cases}\sum_{k=0}^{\infty} \frac{\theta^{2 k}}{(2 k) !} & \text { if } n \text { is even } \\
\sum_{k=0}^{\infty} \frac{|\theta|^{2 k+1}}{(2 k+1) !} & \text { if } n \text { is odd }\end{cases} \\
& = \begin{cases}\cosh (\theta) & \text { if } n \text { is even } \\
\sinh (|\theta|) & \text { if } n \text { is odd }\end{cases}
\end{aligned}
$$

where relation 1 is due to the fact that $\mathbf{E}\left[X^{m}\right]=0$ if $X$ is symmetric about 0 and $m$ is odd, and relation 2 is because $\xi \in[-1,1]$.

We also required Lemma 2 , which considers the uncertainty set-dependent natural upper bound on $\left\|\delta_{i}\right\|_{1}$, represented by $\delta_{i}^{(1)}$. 
Lemma 2. Let $f_{j}: X \rightarrow Y$, where $X=\left\{x_{j} \in \mathbb{R}: 0 \leq x^{L} \leq x_{j} \leq x^{U}\right\}$ and $Y=$ $\{y \in \mathbb{R}: 0 \leq y<\infty\}$, be nondecreasing and log-convex over $X, j=1,2, \ldots, n$, with

$$
\frac{f_{1}\left(x_{i}\right)}{f_{1}\left(x_{j}\right)} \geq \frac{f_{2}\left(x_{i}\right)}{f_{2}\left(x_{j}\right)} \geq \cdots \geq \frac{f_{n}\left(x_{i}\right)}{f_{n}\left(x_{j}\right)} \quad \forall(i, j): x_{i}, x_{j} \in X, x_{i} \geq x_{j}
$$

or

$$
\frac{d \ln f_{1}(x)}{d x} \geq \cdots \geq \frac{d \ln f_{n}(x)}{d x} \quad \forall x \in X .
$$

Given that the nonconvex program

$$
\max _{x}\left\{\prod_{j=1}^{n} f_{j}\left(x_{j}\right): \sum_{j=1}^{n} x_{j} \leq x^{\sigma} ; x_{j} \in X, \forall j\right\},
$$

is feasible (i.e., $x^{L} \leq x^{\sigma} / n$ ), then the point $x^{*}=\left(x_{1}^{*}, x_{2}^{*}, \ldots, x_{j}^{*}, \ldots, x_{n}^{*}\right)^{\top}$, where

$$
\begin{aligned}
x_{j}^{*} & = \begin{cases}x^{U} & j \leq \bar{j} \\
x^{m} & j=\bar{j}+1 \\
x^{L} & j \geq \bar{j}+2,\end{cases} \\
\bar{j} & :=\left\lfloor\frac{x^{\sigma}-n x^{L}}{x^{U}-x^{L}}\right\rfloor, \\
x^{m} & :=x^{\sigma}-\bar{j}\left(x^{U}-x^{L}\right)-(n-1) x^{L},
\end{aligned}
$$

is a global maximizer to program (P1).

Proof. See Lemma 2, Guzman et al. (2016a).

Finally, Lemma 3 takes advantage of the characteristics of the ellipsoidal and interval + ellipsoidal sets and involves the constraint $\left\|\delta_{i}\right\|_{2} \leq \delta_{i}^{(2)}$, where $\delta_{i}^{(2)}$ is the natural upper bound on $\left\|\delta_{i}\right\|_{2}$. This is due to the increased tightness of the $\left\|\delta_{i}\right\|_{2}$ constraint relative to $\left\|\delta_{i}\right\|_{1}$ constraint for these sets (Guzman et al., 2016a).

Lemma 3. Let $f: X \rightarrow Y$, where $X=\{x \in \mathbb{R}: 0 \leq x \leq 1\}$ and $Y=\{y \in \mathbb{R}$ : $0<y<\infty\}$, be nondecreasing over $X$, and let the composite function $(f \circ g)(x)$, where $g(x)=\sqrt{x}$, be concave over $X$. Then the program

$$
\max _{x}\left\{\sum_{j} f\left(x_{j}\right): \sum_{j} x_{j}^{2} \leq 1 ; x_{j} \in[0,1], \forall j\right\}
$$

with $j=1,2, \ldots, n$, has an optimal solution $x_{j}^{*}=1 / \sqrt{n}$.

Proof. See Lemma 3, Guzman et al. (2016a). 


\section{Appendix C. Proofs that distributions meet condition $\left(\mathrm{C}_{2}\right)$}

For each distribution in Table A.4, it must be shown that $\Lambda^{*}(\theta)$ meets condition $\left(\mathrm{C}_{2}\right)$, that is, that $\Lambda^{*}(\sqrt{\theta})$ is concave over $\theta>0$. For the symmetric distributions, it must be shown that $\Lambda^{*}(\sqrt{\theta})=\Lambda(\sqrt{\theta})$ is concave over $\theta>0$. For the asymmetric triangular distribution, it must be shown that $\Lambda^{*}(\sqrt{\theta})=\Lambda(g \sqrt{\theta})$ is concave over $\theta>0$. Of course, a function $F(x)$, where $x \in X \subseteq \mathbb{R}$, is concave over $X$ if and only if

$$
\frac{d^{2} F(x)}{d x^{2}} \leq 0 \quad \forall x \in X .
$$

For $F(x)=\Lambda^{*}(\sqrt{\theta})=\ln M^{*}(\sqrt{\theta})$,

$$
\begin{aligned}
\frac{d}{d \theta}\left(\ln M^{*}(\sqrt{\theta})\right) & =\frac{1}{M^{*}(\sqrt{\theta})} \frac{d M^{*}(\sqrt{\theta})}{d \theta} \\
\frac{d^{2}}{d \theta^{2}}\left(\ln M^{*}(\sqrt{\theta})\right) & =\frac{1}{M^{*}(\sqrt{\theta})^{2}}\left[M^{*}(\sqrt{\theta}) \frac{d^{2} M^{*}(\sqrt{\theta})}{d \theta^{2}}-\left(\frac{d M^{*}(\sqrt{\theta})}{d \theta}\right)^{2}\right] .
\end{aligned}
$$

Note that $M(0)=1, d M(\theta) /\left.d \theta\right|_{0}=0$ if the expected value of its corresponding probability distribution is zero, and $M(\theta)$ is convex. Thus, $M^{*}(\theta)$, and $M^{*}(\sqrt{\theta})$ by extension, is nondecreasing over $\theta>0$ due to symmetry of the distribution or due to the parameterization by $g$. Therefore, $M^{*}(\sqrt{\theta})>0, \forall \theta>0$, and an equivalent expression to (C.1) is

$$
M^{*}(\sqrt{\theta}) \frac{d^{2} M^{*}(\sqrt{\theta})}{d \theta^{2}}-\left(\frac{d M^{*}(\sqrt{\theta})}{d \theta}\right)^{2} \leq 0 \quad \forall \theta>0 .
$$

Using an auxiliary variable $u:=\sqrt{\theta}$ :

$$
\begin{aligned}
\frac{d u}{d \theta} & =\frac{1}{2 \sqrt{\theta}}=\frac{1}{2 u} \\
\frac{d M^{*}(\sqrt{\theta})}{d \theta} & =\frac{d u}{d \theta} \frac{d M^{*}(u)}{d u}=\frac{1}{2 u} \frac{d M^{*}(u)}{d u} \\
\frac{d^{2} M^{*}(\sqrt{\theta})}{d \theta^{2}} & =\frac{d u}{d \theta} \frac{d}{d u}\left(\frac{d M^{*}(\sqrt{\theta})}{d \theta}\right)=\frac{1}{2 u} \frac{d}{d u}\left(\frac{1}{2 u} \frac{d M^{*}(u)}{d u}\right) \\
& =\frac{1}{4 u^{2}} \frac{d^{2} M^{*}(u)}{d u^{2}}-\frac{1}{4 u^{3}} \frac{d M^{*}(u)}{d u} .
\end{aligned}
$$

Note that $u>0$; thus, an equivalent expression to (C.2) is

$$
M^{*}(u) \frac{d^{2} M^{*}(u)}{d u^{2}}-\frac{1}{u} M^{*}(u) \frac{d M^{*}(u)}{d u}-\left(\frac{d M^{*}(u)}{d u}\right)^{2} \leq 0 \quad \forall u>0 .
$$


Finally, as an aid to the proofs below, we list the following identities:

$$
\begin{aligned}
\sinh (x) & =\sum_{n=0}^{\infty} \frac{x^{2 n+1}}{(2 n+1) !} \\
\sinh ^{2}(x / 2) & =\frac{\cosh (x)-1}{2}=\sum_{n=1}^{\infty} \frac{2^{-1} x^{2 n}}{(2 n) !} .
\end{aligned}
$$

Standard normal distribution. The standard normal distribution meets condition $\left(\mathrm{C}_{2}\right)$ linearly:

$$
\frac{1}{2}(\sqrt{\theta})^{2}=\frac{\theta}{2}
$$

Rademacher distribution. By relation (C.1), we must prove the second derivative of $\Lambda(\sqrt{\theta})$ is nonpositive over $\theta>0$ :

$$
\frac{d^{2}}{d \theta^{2}}(\ln \cosh (\sqrt{\theta}))=\frac{2 \sqrt{\theta}-\sinh (2 \sqrt{\theta})}{8 \theta^{3 / 2} \cosh ^{2}(\sqrt{\theta})} \stackrel{?}{\leq} 0 \quad \forall \theta>0 .
$$

Multiplying by the denominator (which is positive) and substituting $u:=\sqrt{\theta}$ :

$$
\begin{array}{rlrl}
2 u-\sinh (2 u) \stackrel{?}{\leq} 0 & \forall u>0 \\
\rightarrow & 2 u-\sum_{n=0}^{\infty} \frac{2^{2 n+1} u^{2 n+1}}{(2 n+1) !} \stackrel{?}{\leq} 0 & \forall u>0 \\
\rightarrow & -\sum_{n=1}^{\infty} \frac{2^{2 n+1} u^{2 n+1}}{(2 n+1) !} \leq 0 & \forall u>0 .
\end{array}
$$

Uniform distribution. By relation (C.3) and with $u:=\sqrt{\theta}$, we must prove

$$
\begin{aligned}
&\left(\frac{\sinh (u)}{u}\right)\left[\frac{\left(u^{2}+2\right) \sinh (u)-2 u \cosh (u)}{u^{3}}-\frac{u \cosh (u)-\sinh (u)}{u^{3}}\right] \\
&-\frac{(\sinh (u)-u \cosh (u))^{2}}{u^{4}} \stackrel{?}{\leq} 0 \quad \forall u>0 .
\end{aligned}
$$


Simplifying and multiplying by $u^{4}$ (which is positive over $u>0$ ):

$$
\begin{array}{rlrl} 
& 2 \sinh ^{2}(u)-\frac{u \sinh (2 u)}{2}-u^{2} \stackrel{?}{\leq} 0 & \forall u>0 \\
\rightarrow \quad 2 u^{2}+\sum_{n=2}^{\infty} \frac{2^{2 n} u^{2 n}}{(2 n) !}-\sum_{n=0}^{\infty} \frac{2^{2 n} u^{2 n+2}}{(2 n+1) !}-u^{2} \stackrel{?}{\leq} 0 & \forall u>0 \\
\rightarrow & \sum_{n=1}^{\infty}\left[\frac{2^{2 n+2} u^{2 n+2}}{(2 n+2) !}-\frac{2^{2 n} u^{2 n+2}}{(2 n+1) !}\right] \stackrel{?}{\leq} 0 & \forall u>0 \\
\rightarrow & \sum_{n=1}^{\infty} \frac{2^{2 n} u^{2 n+2}}{(2 n+1) !}-u^{2} \stackrel{?}{\leq} 0 & \forall u>0 \\
\hline & & 4 & \\
\rightarrow & & &
\end{array}
$$

where the last relation holds because $4 /(2 n+2)$ equals 1 at $n=1$ and is less than 1 for $n>1$.

Symmetric triangular distribution. By relation (C.3) and with $u:=\sqrt{\theta}$, we must prove

$$
\begin{array}{r}
\left(\frac{4(\cosh (u)-1)}{u^{2}}\right) \\
\cdot\left[\frac{\left(u^{2}+6\right) \cosh (u)-4 u \sinh (u)-6}{u^{4}}-\frac{\sinh (u)-u^{-1}(2 \cosh (u)-2)}{u^{3}}\right] \\
-\frac{4\left(4 \sinh ^{2}(u / 2)-u \sinh (u)\right)^{2}}{u^{6}} \stackrel{?}{\leq} 0 \quad \forall u>0 .
\end{array}
$$

Simplifying and multiplying by $u^{6} /\left(8 \sinh ^{2}(u / 2)\right)$ (which is positive over $u>0$ ):

$$
\begin{array}{rlrl}
\rightarrow & \sinh ^{2}(u / 2)-u \sinh (u)-u^{2} \stackrel{?}{\leq} 0 & \forall u>0 \\
\rightarrow \quad 2 u^{2}+\sum_{n=2}^{\infty} \frac{4 u^{2 n}}{(2 n) !}-u^{2}-\sum_{n=1}^{\infty} \frac{u^{2 n+2}}{(2 n+1) !}-\sum_{n=0}^{\infty} \frac{u^{2 n+2}}{(2 n+1) !}-u^{2} \stackrel{?}{\leq} 0 & \forall u>0 \\
\rightarrow \quad \sum_{n=1}^{\infty}\left[\frac{4 u^{2 n+2}}{(2 n+2) !}-\frac{u^{2 n+2}}{(2 n+1) !}\right] \stackrel{?}{\leq} 0 & \forall u>0 \\
\rightarrow \quad \sum_{n=1}^{\infty} \frac{u^{2 n+2}}{(2 n+1) !}\left[\frac{4}{2 n+2}-1\right] \leq 0 & \forall u>0
\end{array}
$$

where the last relation holds because $4 /(2 n+2)$ equals 1 at $n=1$ and is less than 1 for $n>1$. 
Asymmetric triangular distribution. We must prove relation (C.3) where $u:=$ $\sqrt{u}$ and $M^{*}(u)=M(g u)$ :

$$
M(g u)=2 \frac{(c-a) \exp (b g u)-(b-a) \exp (c g u)+(b-c) \exp (a g u)}{(b-a)(b-c)(c-a) u^{2}}
$$

with $g=-\operatorname{sgn} c, c \neq 0, \max \{-a, b\}=1$, and $a+b+c=0$. Without loss of generality, we will assume $a=-1$, and thus $b=1-c, c \in(0,0.5)$, and $g=1$ :

$$
M^{*}(u)=\frac{2 \exp (-u(c-1))(c+1)-2 \exp (-u)(2 c-1)+2 \exp (c u)(c-2)}{(2 c-1)(c+1)(c-2) u^{2}} .
$$

Substituting the above form of $M^{*}(u)$ and its derivatives into relation (C.3), and then multiplying the resulting expression by $u^{6}\left(-2 c^{3}+3 c^{2}+3 c-2\right)^{2} \exp (2(c+$ 1)u) (which is positive over $u>0$ ):

$$
\begin{aligned}
& {\left[\left(-8 c^{4}+4 c^{3}+24 c^{2}+4 c-8\right) u^{2}+\left(8 c^{3}-28 c^{2}+28 c-8\right) u-64 c^{2}+160 c-64\right] e^{(3 c+1) u}} \\
& +\left[\left(16 c^{4}-32 c^{3}-12 c^{2}+28 c-8\right) u^{2}+\left(-4 c^{2}+4 c+8\right) u+32 c^{2}-32 c-64\right] e^{(2 c+3) u} \\
& +\left[\left(-8 c^{4}+28 c^{3}-12 c^{2}-32 c+16\right) u^{2}+\left(-8 c^{3}-4 c^{2}+4 c\right) u-64 c^{2}-32 c+32\right] e^{(c+2) u} \\
& +\left[\left(-4 c^{3}+16 c^{2}-16 c\right) u+16 c^{2}-64 c+64\right] e^{(4 c+2) u} \\
& +\left[\left(4 c^{3}+4 c^{2}-4 c-4\right) u+16 c^{2}+32 c+16\right] e^{4 u} \\
& +\left[\left(16 c^{2}-16 c+4\right) u+64 c^{2}-64 c+16\right] e^{2 c u} \stackrel{?}{\leq} 0 \quad \forall u>0 .
\end{aligned}
$$

Regrouping and dividing by 4 :

$$
\begin{aligned}
\alpha(u) & :=(c+1)^{2} e^{4 u}((c-1) u+4) \\
\beta(u) & :=(2-c)^{2} e^{2(2 c+1) u}(-c u+4) \\
\gamma(u) & :=(1-2 c)^{2} e^{2 c u}(u+4) \\
\delta(u) & :=(c+1)(1-2 c) e^{(c+2) u}\left(\left(4-4 c+c^{2}\right) u^{2}+c u+8\right) \\
\epsilon(u) & :=(2-c)(c+1) e^{(2 c+3) u}\left(-(1-2 c)^{2} u^{2}+u-8\right) \\
\zeta(u) & :=(2-c)(1-2 c) e^{(3 c+1) u}\left(-(c+1)^{2} u^{2}-(1-c) u-8\right) \\
\alpha(u) & +\beta(u)+\gamma(u)+\delta(u)+\epsilon(u)+\zeta(u) \stackrel{?}{\leq} 0 \quad \forall u>0,
\end{aligned}
$$

where the only possible negative component of each term is the polynomial in $u$. Note that at the limit $c=0$, this case becomes the symmetric triangular distribution (where the expression is less than or equal to 0 ), while at the limit 
$c=0.5$,

$$
\begin{aligned}
\alpha(u) & =1.5^{2} e^{4 u}(-0.5 u+4) \\
\beta(u) & =1.5^{2} e^{4 u}(-0.5 u+4) \\
\gamma(u) & =0 \\
\delta(u) & =0 \\
\epsilon(u) & =1.5^{2} e^{4 u}(u-8) \\
\zeta(u) & =0 \\
\alpha(u) & +\beta(u)+\gamma(u)+\delta(u)+\epsilon(u)+\zeta(u)=0 .
\end{aligned}
$$

Each expression follows the form $f_{1}(c) \exp \left(f_{2}(c) u\right) p(c, u)$. The relationship can be analyzed over $u>0$ based on the roots of each polynomial $p(c, u)$ in order to identify critical regions which must be checked for positivity. Expressions $\gamma(u)$ and $\delta(u)$ have no real positive roots and are always positive; also note that $f_{2}^{(\gamma)}(c) \in(0,1)$ and $f_{2}^{(\delta)}(c) \in(2,2.5)$. Expression $\beta(u)$ is positive before its one root at $u=4 / c \in(8, \infty)$, and $f_{2}^{(\beta)}(c) \in(2,4)$. Expression $\alpha(u)$ is positive before its one root at $u=4 /(1-c) \in(4,8)$, and $f_{2}^{(\alpha)}(c)=4$. Because of the high $f_{2}^{(\alpha)}(c)$ of $\alpha(u)$, it should be the primary contributor amongst $\alpha(u), \beta(u)$, $\gamma(u)$, and $\delta(u)$; the only comparable $f_{2}(c)$ is $f_{2}^{(\beta)}(c)$, which approaches 4 as $c \rightarrow 0.5$. However, the root of $\beta(u)$ decreases towards 8 as $c$ increases. The expression $\alpha(u)+\beta(u)+\gamma(u)+\delta(u)$ is always negative over $u \in(8, \infty)$ for all $c$. It remains to be seen if $\epsilon(u)$ and $\zeta(u)$ are sufficient to counteract positivity over $(0,8)$. Expression $\zeta(u)$ is always negative, while $\epsilon(u)$ is positive between its two roots, when they exist. Over $c \in(0,(8-\sqrt{2}) / 16) \approx(0,0.412), \epsilon(u)$ has no real roots and is negative, and the full expression can be observed to be negative over $u \in(0,8)$.

Over $c \in((8-\sqrt{2}) / 16,0.5), \epsilon(u)$ has at least one positive root in $(8,16)$ and is positive between its first root and its second root which lies in $(16, \infty)$. Because $\epsilon(u)$ is negative before its first root, the expression $\alpha(u)+\beta(u)+\epsilon(u)$ can be observed to be near zero or negative over $u \in(0,16)$ (an interval which includes the roots of $\alpha(u)$ and $\beta(u)$ ), while the full expression is negative over this interval. In this range of $c$, the full expression is dominated by $\alpha(u), \beta(u)$, and $\epsilon(u)$, which were the only nonzero components at the limit $c \rightarrow 0.5$ and which have the highest exponential coefficients $f_{2}(c)$ approaching 4 (or equaling 4 for $\alpha(u))$. Over $u>16$ and this range of $c, \alpha(u)$ and $\beta(u)$ are negative. Note that the expression $(2-c)(c+1)(u-8)$ is the overestimating tangent line of quadratic function $\exp (-(2 c-3) u) \epsilon(u)$ at $u=0$; as $c \rightarrow 0.5, \exp ((2 c+3) u) \rightarrow$ $\exp (4 u)$. Replacing $f_{1}^{(\epsilon)}(c) p^{(\epsilon)}(c, x)$ with $f_{1}^{(\epsilon)}(c)(u-8)$ represents the worst case for proving nonnegativity of the relationship; even with this replacement, $f_{1}^{(\alpha)}(c) p^{(\alpha)}(c, x)+f_{1}^{(\beta)}(c) p^{(\beta)}(c, x)+f_{1}^{(\epsilon)}(c)(u-8)$ is a linear function which is positive over $u>0$, approaches 0 as $c \rightarrow 0.5$, and increases while $c$ decreases and the difference between $\exp \left(f_{2}^{(\alpha)}(c) u\right)$ and $\exp \left(f_{2}^{(\epsilon)}(c) u\right)$ sharply increases. Meanwhile, $f_{2}^{(\alpha)}=4$ for all values of $c$, ensuring $\alpha(u)$ remains the dominant 
factor between $\alpha(u), \beta(u)$, and $\epsilon(u)$. Even with the overestimating substitution in $\epsilon(u)$, the full expression is then observed to be positive within $u \in(0,6)$, which was already excluded from positivity in the full expression above.

Raised cosine distribution. By relation (C.3) and with $u:=\sqrt{\theta}$, we must prove

$$
\begin{aligned}
&\left(\frac{\pi^{2} \sinh (u)}{u\left(\pi^{2}+u^{2}\right)}\right)\left[\frac{d^{2}}{d u^{2}}\left(\frac{\pi^{2} \sinh (u)}{u\left(\pi^{2}+u^{2}\right)}\right)\right.\left.-u^{-1} \frac{d}{d u}\left(\frac{\pi^{2} \sinh (u)}{u\left(\pi^{2}+u^{2}\right)}\right)\right] \\
&-\left(\frac{d}{d u}\left(\frac{\pi^{2} \sinh (u)}{u\left(\pi^{2}+u^{2}\right)}\right)\right)^{2} \stackrel{?}{\leq} 0 \quad \forall u>0
\end{aligned}
$$

where

$$
\begin{aligned}
\frac{d}{d u}\left(\frac{\pi^{2} \sinh (u)}{u\left(\pi^{2}+u^{2}\right)}\right)= & \frac{\pi^{2}\left[u\left(\pi^{2}+u^{2}\right) \cosh (u)-\left(\pi^{2}+3 u^{2}\right) \sinh (u)\right]}{\left(u\left(\pi^{2}+u^{2}\right)\right)^{2}} \\
\frac{d^{2}}{d u^{2}}\left(\frac{\pi^{2} \sinh (u)}{u\left(\pi^{2}+u^{2}\right)}\right)= & \left(u\left(\pi^{2}+u^{2}\right)\right)^{-3}\left[-\left(6 \pi^{2} u^{5}+8 \pi^{4} u^{3}+2 \pi^{6} u\right) \cosh (u)\right. \\
& \left.+\left(\pi^{2} u^{6}+\left(2 \pi^{4}+12 \pi^{2}\right) u^{4}+\left(\pi^{6}+6 \pi^{4}\right) u^{2}+2 \pi^{6}\right) \sinh (u)\right] .
\end{aligned}
$$

Simplifying and multiplying by $\left(\pi^{-4} u\left(\pi^{2}+u^{2}\right)\right)^{4}$ :

$$
\begin{array}{ll}
-u^{6}-\frac{\sinh (2 u)}{2} u^{5}+\left(6 \sinh ^{2}(u)-2 \pi^{2}\right) u^{4} & \\
-\pi^{2} \sinh (2 u) u^{3}+\left(4 \pi^{2} \sinh ^{2}(u)-\pi^{4}\right) u^{2} & \\
-\frac{\pi^{4} \sinh (2 u)}{2} u+2 \pi^{4} \sinh ^{2}(u) \stackrel{?}{\leq} 0 & \forall u>0 .
\end{array}
$$

We will define the left-hand side of the relation to be $h(u)$ and note that $h(0)=0$. It will be shown that $d h / d u \leq 0$ over $u \geq 0$, and thus $h(u) \leq 0$ over the same domain. The derivatives of $h(u)$ are:

$$
\begin{aligned}
\frac{d h(u)}{d u}= & -2 u\left(u^{2}+\pi^{2}+2\right)\left(3 u^{2}+\pi^{2}\right)+\left(7 u^{4}+2 \pi^{2} u^{2}+3 \pi^{4}\right) \sinh (u) \cosh (u) \\
& -u\left(u^{4}+2\left(\pi^{2}-6\right) u^{2}+\pi^{2}\left(\pi^{2}-4\right)\right) \cosh (2 u) \\
\frac{d^{2} h(u)}{d u^{2}}= & -2\left(15 u^{4}+6\left(3+2 \pi^{2}\right) u^{2}+\left(u^{4}-19 u^{2}+\pi^{2}\left(2 u^{2}-5\right)+\pi^{4}\right) u \sinh (2 u)\right. \\
& \left.-\left(u^{4}-2\left(\pi^{2}-9\right) u^{2}+\pi^{2}\left(2+\pi^{2}\right)\right) \cosh (2 u)+\pi^{2}\left(2+\pi^{2}\right)\right) \\
\frac{d^{3} h(u)}{d u^{3}}= & -24 u\left(5 u^{2}+2 \pi^{2}+3\right)-2\left(3 u^{4}-93 u^{2}-\pi^{2}\left(9-10 u^{2}\right)-\pi^{4}\right) \sinh (2 u) \\
& -4 u\left(u^{4}-21 u^{2}+\pi^{2}\left(2 u^{2}-3\right)+\pi^{4}-18\right) \cosh (2 u) .
\end{aligned}
$$

Note that all three expressions are equal to 0 at $u=0$. Constituents of the third derivative include quadratic and biquadratic polynomials which can be easily 
examined to find their roots. The third derivative is rewritten as

$$
\begin{aligned}
\frac{d^{3} h(u)}{d u^{3}} & =p_{1}(u)+p_{2}(u)+p_{3}(u) \\
p_{1}(u) & :=-24 u\left(5 u^{2}+\left(2 \pi^{2}+3\right)\right) \\
p_{2}(u) & :=-2\left(3 u^{4}+\left(10 \pi^{2}-93\right) u^{2}-\left(\pi^{4}+9 \pi^{2}\right)\right) \sinh (2 u) \\
p_{3}(u) & :=-4 u\left(u^{4}+\left(2 \pi^{2}-21\right) u^{2}+\left(\pi^{4}-3 \pi^{2}-18\right)\right) \cosh (2 u) .
\end{aligned}
$$

Expressions $p_{1}(u)$ and $p_{3}(u)$ only have real roots at $u=0$, and thus are both negative over $u>0$. Expression $p_{2}(u)$ has real roots at 0 and

$$
\pm \sqrt{\frac{1}{6}\left(93-10 \pi^{2}+\sqrt{8649-1752 \pi^{2}+112 \pi^{4}}\right)} \approx \pm 2.64 .
$$

The first derivative of $p_{2}(u)$ is negative at the positive root (which we will call $\left.r_{+}\right)$, and thus

$$
\begin{aligned}
& p_{2}(u) \geq 0 \\
& u \in\left[0, r_{+}\right] \\
& p_{2}(u)<0 \\
& u>r_{+} \text {. }
\end{aligned}
$$

Therefore, $d^{3} h / d u^{3}$ only has a positive addend (coming from $p_{2}(u)$ ) over the range $\left(0, r_{+}\right)$, and can be observed to be strictly negative over this finite range; this was confirmed here with very fine discretizations of the region. Thus, $d^{3} h / d u^{3}$ is nonpositive over $u \geq 0$. Following the implications of this to $h(u)$ :

$$
\begin{aligned}
& \left.\begin{array}{rl}
\frac{d^{3} h(u)}{d u^{3}} & \leq 0, \forall u \geq 0 \\
\left.\frac{d^{2} h(u)}{d u^{2}}\right|_{0} & =0
\end{array}\right\} \Longrightarrow \frac{d^{2} h(u)}{d u^{2}} \leq 0, \forall u \geq 0 \\
& \left.\begin{array}{rl}
\frac{d^{2} h(u)}{d u^{2}} & \leq 0, \forall u \geq 0 \\
\left.\frac{d h(u)}{d u}\right|_{0} & =0
\end{array}\right\} \Longrightarrow \frac{d h(u)}{d u} \leq 0, \forall u \geq 0 \\
& \left.\begin{array}{l}
\frac{d h(u)}{d u} \leq 0, \forall u \geq 0 \\
h(0)=0
\end{array}\right\} \Longrightarrow h(u) \leq 0, \forall u \geq 0 .
\end{aligned}
$$




\section{References}

Averbakh, I., Zhao, Y.-B., 2008. Explicit reformulations for robust optimization problems with general uncertainty sets. SIAM Journal on Optimization 18 (4), $1436-1466$.

Ben-Tal, A., Boyd, S., Nemirovski, A., 2006. Extending scope of robust optimization: Comprehensive robust counterparts of uncertain problems. Mathematical Programming 107 (1), 63-89.

Ben-Tal, A., El Ghaoui, L., Nemirovski, A., 2009. Robust optimization. Princeton University Press.

Ben-Tal, A., Goryashko, A., Guslitzer, E., Nemirovski, A., 2003. Adjustable robust solutions of uncertain linear programs. Mathematical Programming 99 (2), 351-376.

Ben-Tal, A., Nemirovski, A., 1998. Robust convex optimization. Mathematics of Operations Research 23 (4), 769-805.

Ben-Tal, A., Nemirovski, A., 1999. Robust solutions of uncertain linear programs. Operations research letters 25 (1), 1-13.

Ben-Tal, A., Nemirovski, A., 2000. Robust solutions of linear programming problems contaminated with uncertain data. Mathematical programming 88 (3), 411-424.

Ben-Tal, A., Nemirovski, A., 2007. Selected topics in robust convex optimization. Mathematical Programming 112 (1), 125-158.

Bertsimas, D., Brown, D. B., 2009. Constructing uncertainty sets for robust linear optimization. Operations Research 57 (6), 1483-1495.

Bertsimas, D., Brown, D. B., Caramanis, C., 2011. Theory and applications of robust optimization. SIAM Review 53 (3), 464-501.

Bertsimas, D., Goyal, V., 2012. On the approximability of adjustable robust convex optimization under uncertainty. Mathematical Methods of Operations Research 77 (3), 323-343.

Bertsimas, D., Sim, M., 2004. The price of robustness. Operations research $52(1), 35-53$.

Boyd, S., Vandenberghe, L., 2004. Convex optimization. Cambridge university press.

Chen, X., Zhang, Y., 2009. Uncertain linear programs: Extended affinely adjustable robust counterparts. Operations Research 57 (6), 1469-1482.

El Ghaoui, L., Lebret, H., 1997. Robust solutions to least-squares problems with uncertain data. SIAM Journal on Matrix Analysis and Applications 18 (4), 1035-1064. 
El Ghaoui, L., Oustry, F., Lebret, H., 1998. Robust solutions to uncertain semidefinite programs. SIAM Journal on Optimization 9 (1), 33-52.

Floudas, C. A., 1995. Nonlinear and Mixed-Integer Optimization: Fundamentals and Applications. Oxford University Press.

Floudas, C. A., Gümüs, Z. H., Ierapetritou, M. G., 2001. Global optimization in design under uncertainty: feasibility test and flexibility index problems. Industrial \& Engineering Chemistry Research 40 (20), 4267-4282.

Floudas, C. A., Niziolek, A. M., Onel, O., Matthews, L. R., 2016. Multi-scale systems engineering for energy and the environment: Challenges and opportunities. AIChE Journal 62 (3), 602-623.

Gabrel, V., Murat, C., Thiele, A., 2014. Recent advances in robust optimization: An overview. European Journal of Operational Research 235 (3), 471 - 483.

Gounaris, C. E., Misener, R., Floudas, C. A., 2009. Computational comparison of piecewise- linear relaxations for pooling problems. Industrial \& Engineering Chemistry Research 48 (12), 5742-5766.

Gounaris, C. E., Wiesemann, W., Floudas, C. A., 2013. The robust capacitated vehicle routing problem under demand uncertainty. Operations Research $61(3), 677-693$.

Guzman, Y. A., Matthews, L. R., Floudas, C. A., 2016a. New a priori and a posteriori probabilistic bounds for robust counterpart optimization: I. Unknown probability distributions. Computers \& Chemical Engineering 84, 568-598.

Hoeffding, W., 1963. Probability inequalities for sums of bounded random variables. Journal of the American Statistical Association 58 (301), 13-30.

Janak, S. L., , Floudas, C. A., Kallrath, J., Vormbrock, N., 2006. Production scheduling of a large-scale industrial batch plant. II. reactive scheduling. Industrial \& Engineering Chemistry Research 45 (25), 8253-8269.

Janak, S. L., Lin, X., Floudas, C. A., 2007. A new robust optimization approach for scheduling under uncertainty: II. Uncertainty with known probability distribution. Computers \& chemical engineering 31 (3), 171-195.

Kang, S.-C., Brisimi, T. S., Paschalidis, I. C., 2013. Distribution-dependent robust linear optimization with applications to inventory control. Annals of Operations Research, 1-35.

Kotz, S., Kozubowski, T., Podgorski, K., 2012. The Laplace distribution and generalizations: a revisit with applications to communications, economics, engineering, and finance. Springer Science \& Business Media.

Lappas, N. H., Gounaris, C. E., 2016. Multi-stage adjustable robust optimization for process scheduling under uncertainty. AIChE Journal 62 (5), 16461667. 
Li, J., Misener, R., Floudas, C. A., 2012a. Scheduling of crude oil operations under demand uncertainty: A robust optimization framework coupled with global optimization. AIChE Journal 58 (8), 2373-2396.

Li, Z., Ding, R., Floudas, C. A., 2011. A comparative theoretical and computational study on robust counterpart optimization: I. Robust linear optimization and robust mixed integer linear optimization. Industrial \& Engineering Chemistry Research 50 (18), 10567-10603.

Li, Z., Tang, Q., Floudas, C. A., 2012b. A comparative theoretical and computational study on robust counterpart optimization: II. Probabilistic guarantees on constraint satisfaction. Industrial \& Engineering Chemistry Research 51 (19), 6769-6788.

Li, Z., Floudas, C. A., 2014. A comparative theoretical and computational study on robust counterpart optimization: III. Improving the quality of robust solutions. Industrial \& Engineering Chemistry Research 53 (33), 13112-13124.

Lin, X., Janak, S. L., Floudas, C. A., 2004. A new robust optimization approach for scheduling under uncertainty: I. Bounded uncertainty. Computers \& chemical engineering 28 (6), 1069-1085.

Misener, R., Floudas, C. A., 2010. Global optimization of large-scale pooling problems: Quadratically constrained MINLP models. Industrial \& Engineering Chemistry Research 49 (11), 5424-5438.

Misener, R., Floudas, C. A., 2012. Global optimization of mixed-integer quadratically-constrained quadratic programs (MIQCQP) through piecewiselinear and edge-concave relaxations. Mathematical Programming 136 (1), $155-182$.

Misener, R., Floudas, C. A., 2013. GloMIQO: Global mixed-integer quadratic optimizer. Journal of Global Optimization 57 (1), 3-50.

Misener, R., Floudas, C. A., 2014a. ANTIGONE: Algorithms for coNTinuous/Integer Global Optimization of Nonlinear Equations. Journal of Global Optimization 59 (2-3), 503-526.

Misener, R., Floudas, C. A., 2014b. A framework for globally optimizing mixedinteger signomial programs. Journal of Optimization Theory and Applications 161 (3), 905-932.

Misener, R., Smadbeck, J. B., Floudas, C. A., 2015. Dynamically generated cutting planes for mixed-integer quadratically constrained quadratic programs and their incorporation into GloMIQO 2. Optimization Methods and Software 30 (1), 215-249.

Misener, R., Thompson, J. P., Floudas, C. A., 2011. APOGEE: Global optimization of standard, generalized, and extended pooling problems via linear and logarithmic partitioning schemes. Computers \& Chemical Engineering $35(5), 876-892$. 
Nemirovski, A., Shapiro, A., 2007. Convex approximations of chance constrained programs. SIAM Journal on Optimization 17 (4), 969-996.

Paschalidis, I., Kang, S., Li, K., 2008. Distribution-dependent robust linear optimization with asymmetric uncertainty and application to optimal control. In: Proceedings of the 17th World Congress IFAC. pp. 10069-10074.

Pistone, G., Wynn, H. P., 1999. Finitely generated cumulants. Statistica Sinica 9 (4), 1029-1052.

Ravindran, A. R., 2008. Operations research methodologies. CRC Press.

Slater, M., Nov. 1950. Lagrange multipliers revisited: A contribution to nonlinear programming. Cowles Commission Discussion Paper, Math. 403.

Soyster, A. L., 1973. Convex programming with set-inclusive constraints and applications to inexact linear programming. Operations research 21 (5), 11541157 .

Verderame, P. M., Floudas, C. A., 2009a. Operational planning of large-scale industrial batch plants under demand due date and amount uncertainty: I. robust optimization framework. Industrial \& engineering chemistry research 48 (15), 7214-7231.

Verderame, P. M., Floudas, C. A., 2009b. Operational planning of large-scale industrial batch plants under demand due date and amount uncertainty: II. Conditional value-at-risk framework. Industrial \& engineering chemistry research 49 (1), 260-275. 